\title{
Quantitative analysis of the spontaneous activity and response profiles of odorant receptor neurons in larval Xenopus laevis using the cell-attached patch-clamp technique
}

\author{
INAUGURAL-DISSERTATION \\ zur Erlangung des Doktorgrades \\ der Medizinischen Fakultät der \\ Georg-August-Universität zu Göttingen
}

vorgelegt von

Rodi Topci

aus

Merikis (Syrien)

Göttingen 2019 
Die Vorliegende Dissertation wurde im Institut für Neurophysiologie und zelluläre Biophysik im Zentrum Physiologie und Pathophysiologie in Göttingen im Zeitraum von Februar 2014 bis September 2016 unter der Betreuung von Prof. Dr. rer. nat. Dr. med. Detlev Schild angefertigt.

Dekan:

Referent/in

Ko-Referentin:

Drittreferentin:
Prof. Dr. med. W. Brück

Prof. Dr. rer. nat. Dr. med. D. Schild

Prof. Dr. Tina Pangrsic Vilfan

Prof. Dr. Margarete Schön

Datum der mündlichen Prüfung: 24.06.2020 
Hiermit erkläre ich, die Dissertation mit dem Titel "Quantitative analysis of the spontaneous activity and response profiles of odorant receptor neurons in larval Xenopus laevis using the cell-attached patch-clamp technique" eigenständig angefertigt und keine anderen als die von mir angegebenen Quellen und Hilfsmittel verwendet zu haben.

Göttingen, den 


\section{Table of contents}

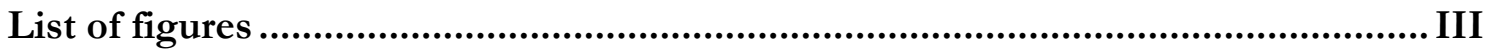

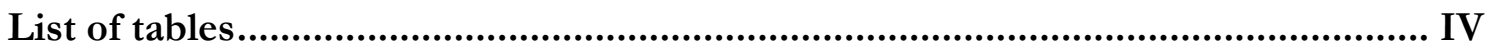

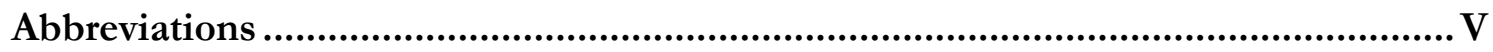

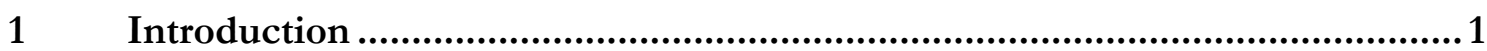

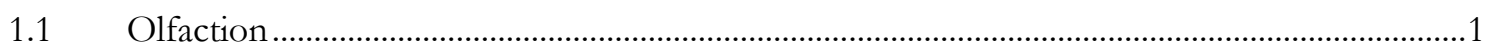

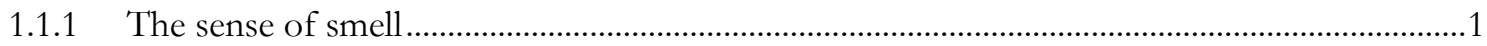

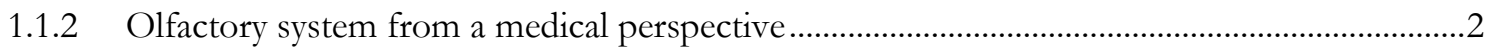

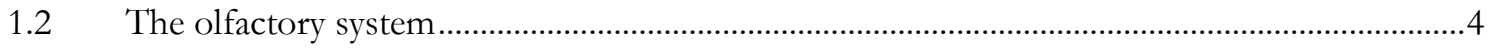

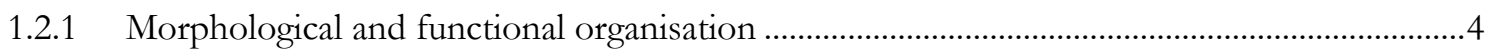

1.2.2 Signal transduction and transformation in ORNs of larval Xenopus laevis .................................

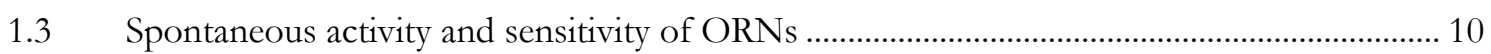

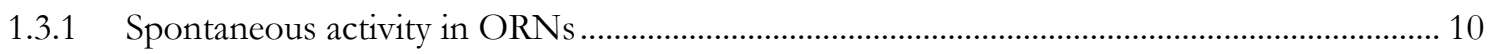

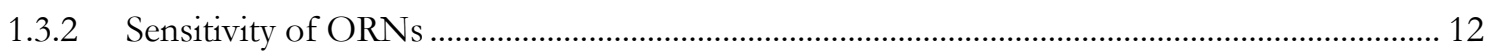

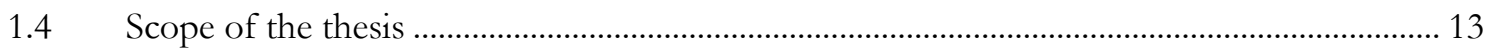

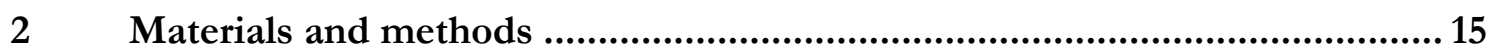

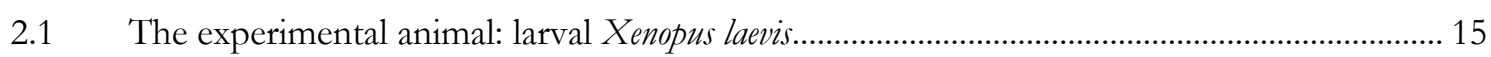

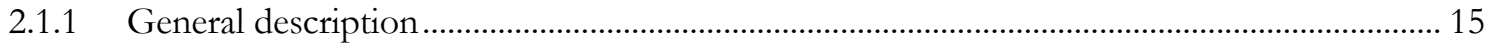

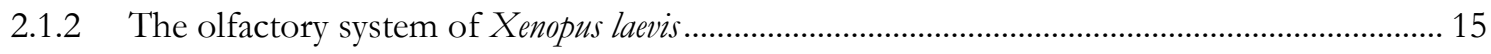

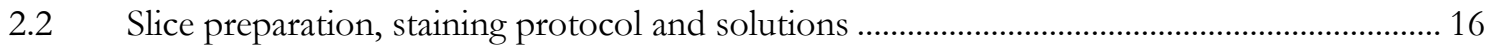

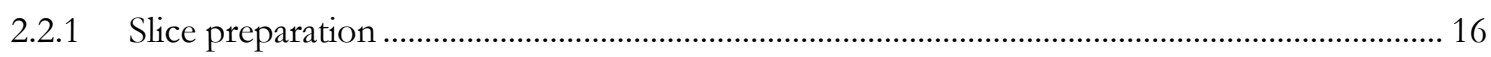

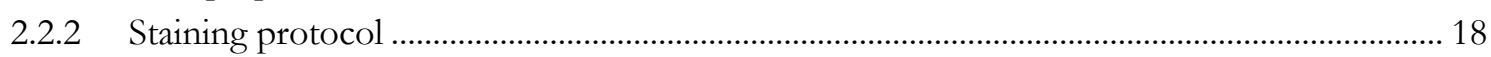

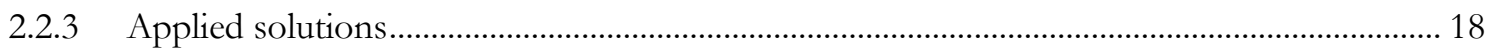

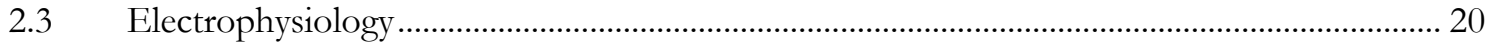

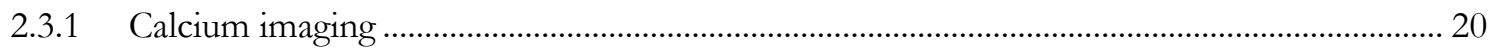

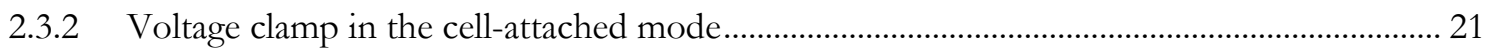

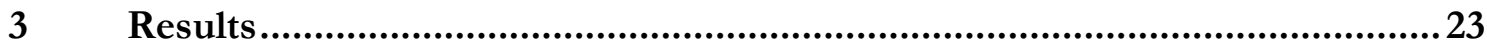

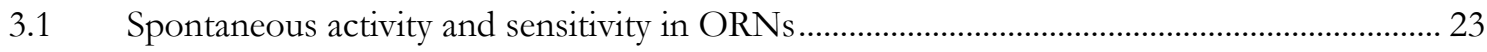

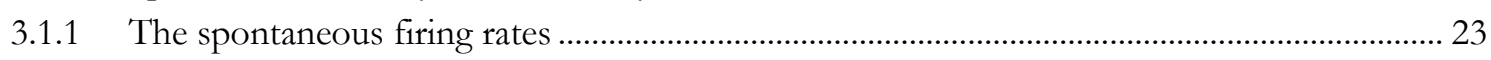

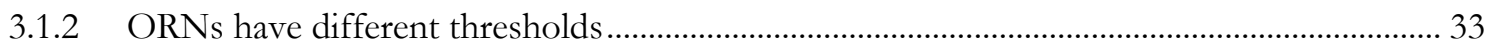

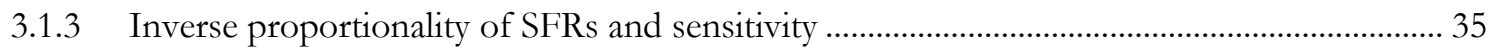

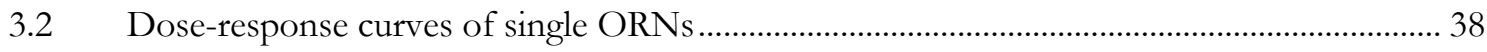

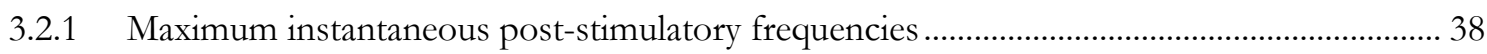

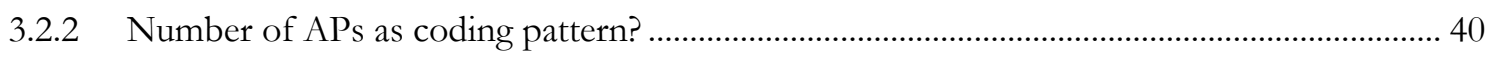

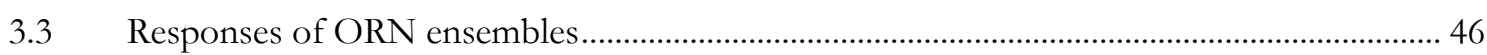

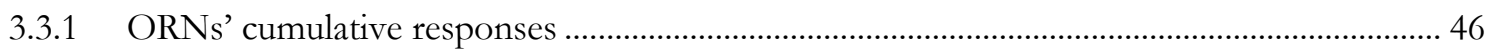

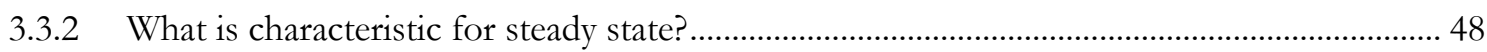




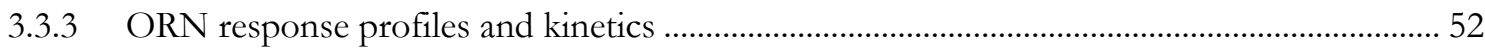

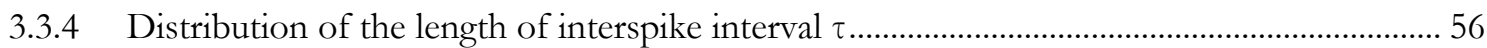

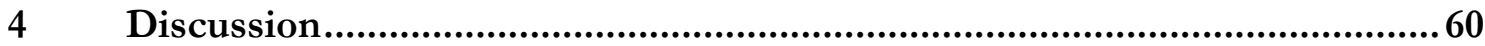

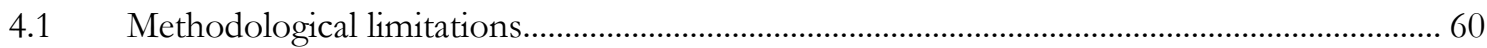

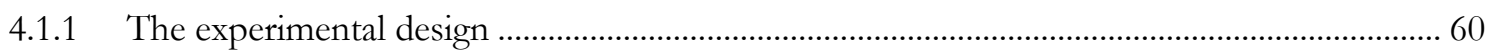

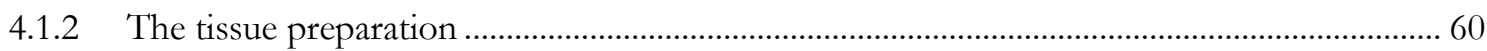

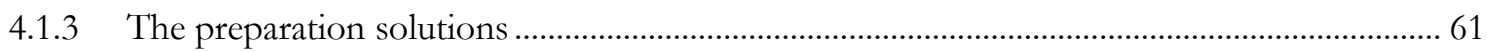

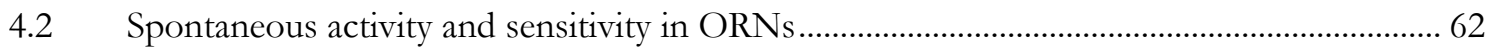

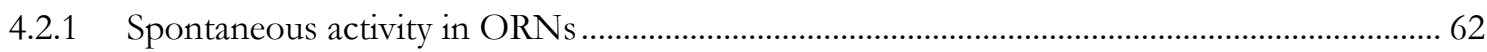

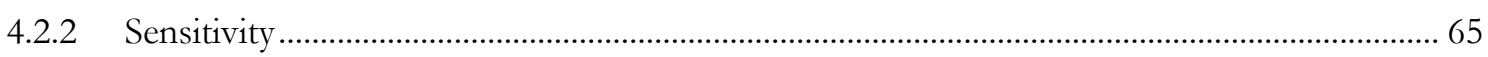

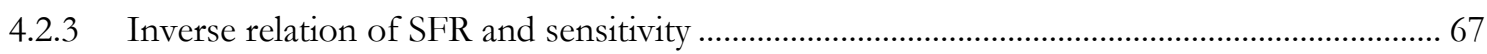

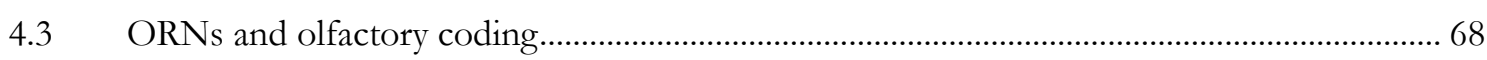

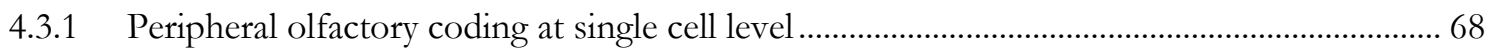

4.3.2 Peripheral olfactory coding in neuronal ensembles and stationarity ........................................ 70

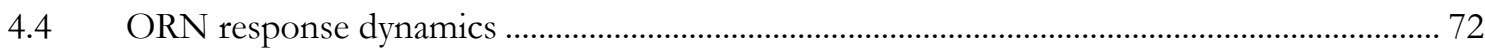

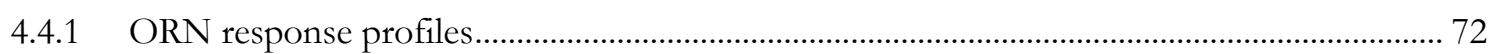

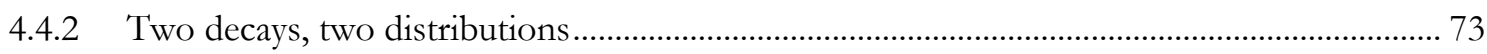

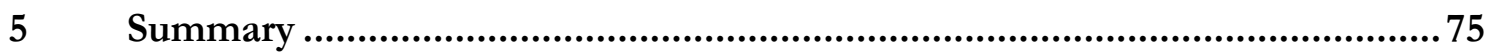

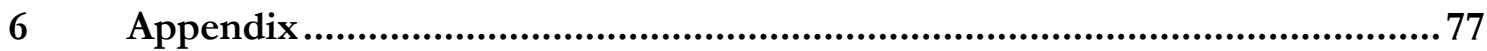

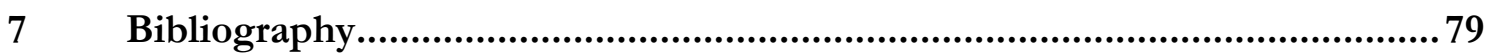




\section{List of figures}

Figure 1 Schematic representation of the olfactory epithelium and olfactory bulb.................6

Figure 2 Slice preparation of larval Xenopus laevis ............................................................. 17

Figure 3 Calcium imaging and single-cell patching of ORNs .............................................. 21

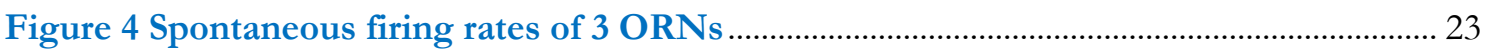

Figure 5 Distribution of averaged spontaneous firing rates .................................................. 25

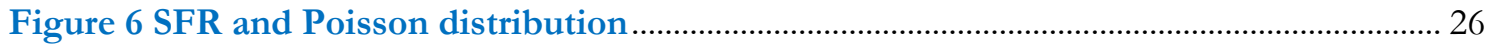

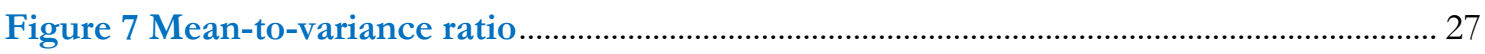

Figure 8 ORNs with high variance of spontaneous activity.................................................. 28

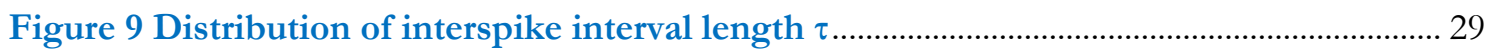

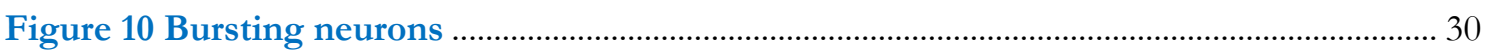

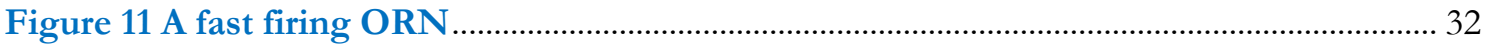

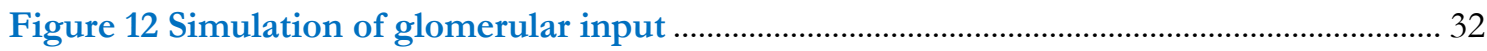

Figure 13 Stimulus-induced responses in an arginine-sensitive ORN.................................. 33

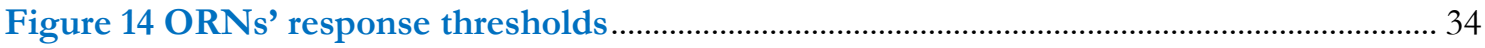

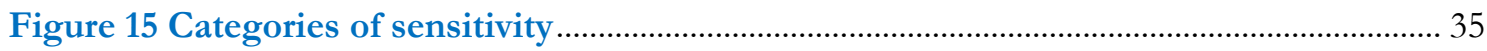

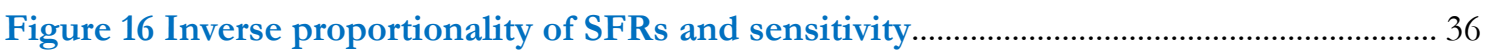

Figure 17 Ranges of maximum instantaneous frequencies .................................................. 38

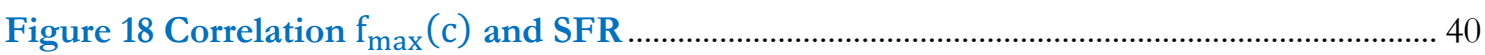

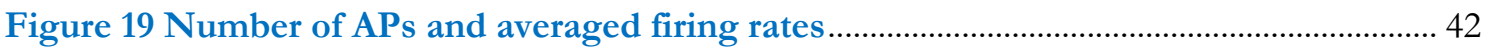

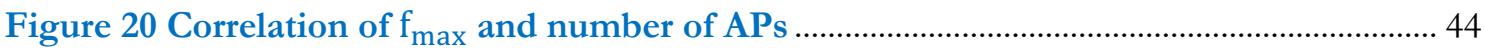

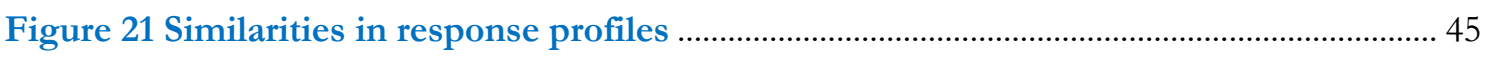

Figure 22 Cumulative responses of 9 ORNs to different stimulus concentrations ............... 47

Figure 23 Non-stationarity at high stimulus concentrations ................................................. 49

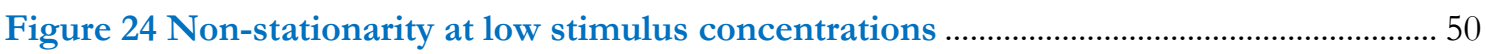

Figure 25 Non-stationarity at threshold concentrations ....................................................... 51

Figure 26 Stimulus-induced instantaneous frequencies over time........................................ 53

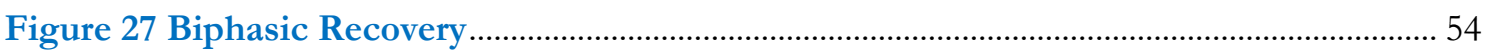

Figure 28 Stimulus-induced patterns of interspike intervals in a single ORN..................... 57

Figure 29 The distribution of interspike intervals in 9 different ORNs................................ 58

Figure 30 Color-coded 2D map of interspike interval subsequences .................................... 59

Figure 31 Sensitivity and specificity of glomeruli ................................................................. 72

Figure 32 ORNs sensitive to arginine, methionine and alanine ............................................ 77

Figure 33 Ratios of $\mathrm{f}_{\max }$ and aSFR in low and highly sensitive ORNs................................ 78 


\section{List of tables}

Table 1 List of amino acid subgroups and single amino acids............................................... 19

Table 2 Composition of the Ringer's solution ....................................................................... 19

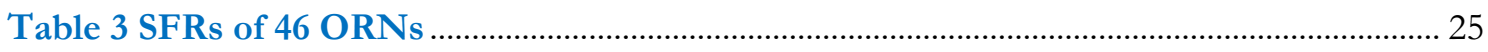

Table 4 Averaged instantaneous post-stimulation frequencies.................................................. 39

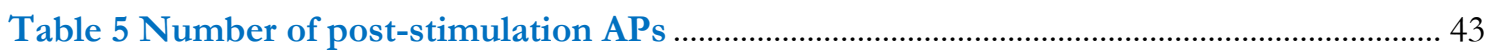

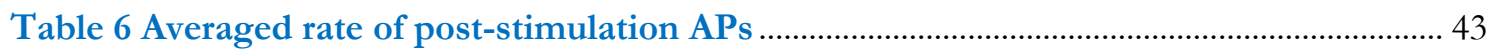

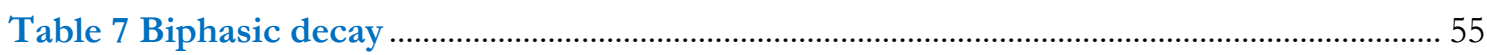

Table 8 Ratios of $\mathrm{f}_{\max }$ and aSFR in low and highly sensitive ORNs .................................. 78 


\section{Abbreviations}

AC III

AOB

$\mathrm{AP}(\mathrm{s})$

BC

$\mathrm{CaCC}$

cAMP

$\mathrm{CNG}$

DMSO

ffORN

$G_{\text {olf }}$

$\mathrm{HCN}$

hsORN

imORN

KST

lsORN

$\mathrm{MOB}$

MOE

$\mathrm{OB}$

OE

OR

ORN(s)

$\mathrm{SC}(\mathrm{s})$

sfORN

VNO adenylyl cyclase III

accessory olfactory bulb

action potential(s)

basal cell

calcium-activated chloride channel

cyclic adenosine monophosphate

cyclic nucleotide-gated channel

dimethyl sulfoxide

fast firing olfactory receptor neuron

olfactory-specific guanosine triphosphate-binding protein

hyperpolarization-activated cyclic nucleotide-gated cation channel

high-sensitive olfactory receptor neuron

intermediately firing olfactory receptor neuron

Kolmogorov-Smirnov test

low-sensitive olfactory receptor neuron

main olfactory bulb

main olfactory epithelium

olfactory bulb

olfactory epithelium

olfactory receptor

olfactory receptor neuron(s)

sustentacular cell(s)

slow firing olfactory receptor neuron

vomeronasal organ 


\section{Introduction}

\subsection{Olfaction}

\subsubsection{The sense of smell}

Olfaction describes the ability to detect a vast range of different chemical molecules in the environment. It enables the organism to perceive relevant air-borne or water-soluble components from a long or short distance. An intact and more sensitive olfactory system presumably gave a survival advantage to certain species over others. This may explain the fact that this sensory system is being found across phyla from invertebrates like insects to vertebrates (fish, amphibians, reptiles, birds and mammals).

The ability to sense chemical molecules is a feature of unicellular organisms among prokaryotes as well as eukaryotes. Bacteria like Escherichia coli, for example, express transmembrane chemoreceptors, known as methyl-accepting chemotaxis proteins. The binding of ligands like sugars or amino acids controls the direction of the flagellar motor through a sophisticated molecular signaling and thereby allows the bacteria's targeted motion (Sourjik and Wingreen 2012). A prominent example in multicellular organisms is the migration of leukocytes which detect chemical gradients of chemokines via members of the seven-transmembrane spanning G protein-coupled receptors (GPCR) family; this enables leukocytes to find the inflammation site (Bloes et al. 2015).

However, olfaction does not only involve ligand-receptor interactions but requires a complex chemosensory system. It is built upon neurons - olfactory receptor neurons (ORNs) - that are directly exposed to the environment and express olfactory receptors (ORs) in order to detect corresponding ligands. Such ligands are mostly low molecular organic compounds consisting of carbohydrates with diverse functional groups, e.g. alcohols or amino acids (Firestein 2001). Binding such odorants will ignite the signal transduction cascade that, in turn, opens ion channels which finally leads to crossing the membrane threshold potential for action potential generation. The odor information is then conveyed via the axon terminals of primary sensory neurons to second-order neurons in the olfactory bulb. There, ORN axons form spheroid structures called glomeruli and make synapses with mitral and tufted cells (Nezlin and Schild 2000; Firestein 2001). Finally, that information will be transmitted to higher brain centers for further information processing, decoding and odor recognition. 
The olfactory system is crucial for the survival of different animal species as it is involved in major behavioral aspects of life such as food search behavior, food intake (Reinhard et al. 2004; Zielinski and Hara 2006; Buehlmann et al. 2014) and reproduction (Spehr et al. 2006; Polese et al. 2015). In species with complex social organization like mammals it plays an outstanding role in socio-sexual matters (Martín-Sánchez et al. 2015), maternal-offspring interaction (Corona and Lévy 2015) and recognition of conspecifics and territory fights (Brennan and Kendrick 2006; Stopka et al. 2007; Martín-Sánchez et al. 2015).

Nevertheless, the significance of the mammalian olfactory system was a long time underestimated because humans as well as other higher primates predominantly rely on other senses when interacting with their environment and conspecifics. The discovery of olfactory receptor protein genes (Buck and Axel 1991), however, was a starting point of numerous studies aiming to understand the olfactory system. Although much progress has been done since then, further research efforts are necessary to unravel the complex organisation und function of the olfactory system.

\subsubsection{Olfactory system from a medical perspective}

Although humans and other higher primates possess a non-functional vomeronasal organ (Meredith 2001) and the majority of olfactory receptor genes being pseudogenes (Nei et al. 2008), studying olfaction remains clinically relevant.

Disorders of the olfactory system have been found in various diseases, especially in neurodegenerative diseases (Hawkes 2003). Hyposmia appears to be one of the first symptoms in patients with Alzheimer's disease and could predict the incidence of amnestic mild cognitive impairment and the conversion of the latter to Alzheimer's disease (Roberts et al. 2016). Olfactory tests can therefore play a screening role for amnestic mild cognitive impairment and its progression to Alzheimer's disease (Roberts et al. 2016). Another prominent neurodegenerative disease with olfactory abnormalities is idiopathic Parkinson's disease (Doty 2012). Interestingly, the degree of sensory loss in idiopathic Parkinson's disease is higher than in other related movement disorders, such as progressive supranuclear palsy, multiple system atrophy, corticobasal degeneration (Wenning et al. 1995; Goldstein and Sewell 2009) or in essential tremor (Busenbark et al. 1992; Shah et al. 2008). In a certain study hyposmia occurred in $>90 \%$ of patients with idiopathic Parkinson's disease (Doty et al. 1988). That makes olfactory testing a valuable tool for a differential diagnosis (Goldstein and Sewell 2009). 
Alterations of olfactory system are not only observed in neurodegenerative diseases but also in psychiatric disorders, particularly in patients with major depression (Pause et al. 2001; Atanasova et al. 2008) and schizophrenia (Moberg et al. 1999). Patients that are diagnosed with schizophrenia or a schizoaffective disorder show deficits in affective and cognitive functions. Several studies have shown that schizophrenia is associated with olfactory dysfunctions like a loss of sensitivity in odor discrimination and detection threshold, an impaired odor memory and altered hedonic judgments (Atanasova et al. 2008; Kamath et al. 2014). Furthermore, unpleasant olfactory hallucinations are a typical positive symptom of schizophrenia and can predict the severity of the disease (Stevenson et al. 2011)

Taken together, these studies demonstrated that alterations of olfactory system are important symptoms in neurological and psychiatric disorders with high prevalence in the population. Understanding the pathological mechanisms underlying the olfactory symptoms can help to understand the pathophysiology of neurodegeneration in other neuronal populations. A major advantage of olfaction research is the accessibility to neuronal cells.

Another functional system that is closely linked to the olfactory system is the endocrine system as both are involved in nutrition and energy status (Baly et al. 2007; Prud'homme et al. 2009; Savigner et al. 2009). With the high incidence of obesity in modern societies, and cardiovascular diseases being the most prevalent cause of death (Nichols et al. 2014), exploring the role of olfaction in the context of human feeding behavior becomes all the more relevant.

Finally, there are important medical arguments not only to investigate the olfactory system of humans but of other species too, namely insects and arachnids. Arthropods are wellknown vectors for several diseases, among them, Anopheles mosquitoes transmitting malaria (Takken and Knols 1999), Aedes aegypti flies transmitting yellow fever and Dengue's fever (Nene et al. 2007) and phlebotomine sandflies transmitting leishmaniasis (Kamhawi 2006). The impact of zoonoses was assessed by the WHO in their world report on malaria 2015 (World Health Organization 2016). Therein, the WHO reported 212 million cases of malaria, among which 429.000 were lethal and $70 \%$ of the deceased patients were aged less than 5 years. The overall costs for malaria control and elimination were estimated at $\$ 2.9$ billion for 2015 . Other alarming numbers are the cases of yellow fever and dengue amounting to 200,000 and 50 million individuals each year, respectively (Nene et al. 2007). These numbers illustrate the devastating implications of these diseases 
on global health. They are transmitted by vectors that predominantly use their olfactory system to locate hosts, mating and selecting egg-laying sites (Foster and Takken 2004; Zwiebel and Takken 2004; Smallegange et al. 2010). In colder regions like Europe, Lyme disase (Hubálek 2009) and tick-borne encephalitis are being transmitted by ticks of the Ixodes genus (Lindquist and Vapalahti 2008).

Thus, there is a great need of elucidating the cellular mechanisms underlying the insects' olfactory behavior and thereby finding strategies targeting their olfactory systems to prevent the spread of insect-borne diseases.

\subsection{The olfactory system}

\subsubsection{Morphological and functional organisation}

The olfactory system is supposed to be first developed in aquatic animals as the beginning of life took place in the oceans. The evolution of the olfactory system induced the development of morphologically and functionally diverse systems among different species in animal kingdom being adapted to the environmental conditions of the animal's life.

The olfactory system enables the organism to detect selected molecules from their environment. These molecules serve as biological markers and inform the animal about food sources, possible mating partners or predators (see section 1.1.1). Most volatile molecules that inform about food sources are referred to as odorants. Other molecules transmitting information about conspecifics and being mostly non-volatile excreted or secreted by individuals of the same species are called pheromones (Tirindelli et al. 2009). Odorants and pheromones do not only differ chemically and in their biological relevance but they are also recognized by different receptor types and sub-compartments of the olfactory epithelium and are processed in distinct regions of the central nervous system.

The molecules are recognized by a complex inventory of olfactory receptor proteins being located on the most apical part of the olfactory receptor neurons' (ORN) prolongations which are directly exposed to the environment. Inherently, there is a major difference in the olfactory systems of phyla like e.g. vertebrates and arthropods. In insects, olfactory receptors are mostly organised as heteromeric ligand-gated ion channels (Sato et al. 2008; Wicher et al. 2008), in contrast to mammalian receptors which mostly possess a metabotropic signaling via the cyclic adenosine monophosphate cAMP-pathway. Insect olfactory receptors act in an ionotropic signaling way, with metabotropic autoregulation (Nakagawa and Vosshall 2009; Silbering and Benton 2010). For a detailed analysis of the 
olfactory system in insects see Szyszka and Galizia (2015). The scope of this thesis is to study the vertebrate olfactory system of larval Xenopus laevis, and particularly to record the activity patterns of ORNs.

Morphology and function of the olfactory epithelium. The vertebrate olfactory system consists of the olfactory epithelium (OE) which is connected via the olfactory nerve to the olfactory bulb (OB) (Figure 1). In the OB, ORN axons form spheroid structures that are called glomeruli where the axon terminals of the olfactory receptor neurons build synapses with mitral and tufted cells (Firestein 2001; Mombaerts 2006). These cells project to different regions of the central nervous system. The OE represents a minor part of the respiratory epithelium in the nasal cavity of mammals. In fully terrestrial animals the $\mathrm{OE}$ is typically composed of different sub-compartments; the main olfactory epithelium (MOE) (Getchell 1986), the vomeronasal organ (VNO) (Zancanaro 2014), the septal organ of Masera (Ma et al. 2003) and the Grüneberg Ganglion (Grüneberg 1973). The VNO and possibly the septal organ as well as the Grüneberg ganglion are involved in the detection of pheromones (Francia et al. 2014) whereas the MOE harbours ORNs which detect odorants. The MOE is a pseudostratified neuroepithelium that mainly consists of 3 cell types; the ORNs, the sustentacular cells (SCs) and the basal cells (BCs) (Getchell 1986; Lancet 1986; Hansen et al. 1998; Schild and Restrepo 1998). An ORN is a bipolar neuron with a round- or ellipsoid-shaped soma and a single apical dendrite terminating in a dendritic knob. From this knob a various number of cilia, on average a number of 5 to 40 varying in different species, end at the mucosal surface (Schild and Restrepo 1998). However, there are also a second type of ORNs bearing microvilli instead of kinocilia in adult and larval Xenopus laevis (Hansen et al. 1998). The basal part of the soma gives rise to a single axon which branches and targets mostly 2-3 glomeruli in the olfactory bulb of larval Xenopus laevis. Every branch of the axon subdivides again into 2 sub-branches before entering a glomerulus (Nezlin and Schild 2005). 


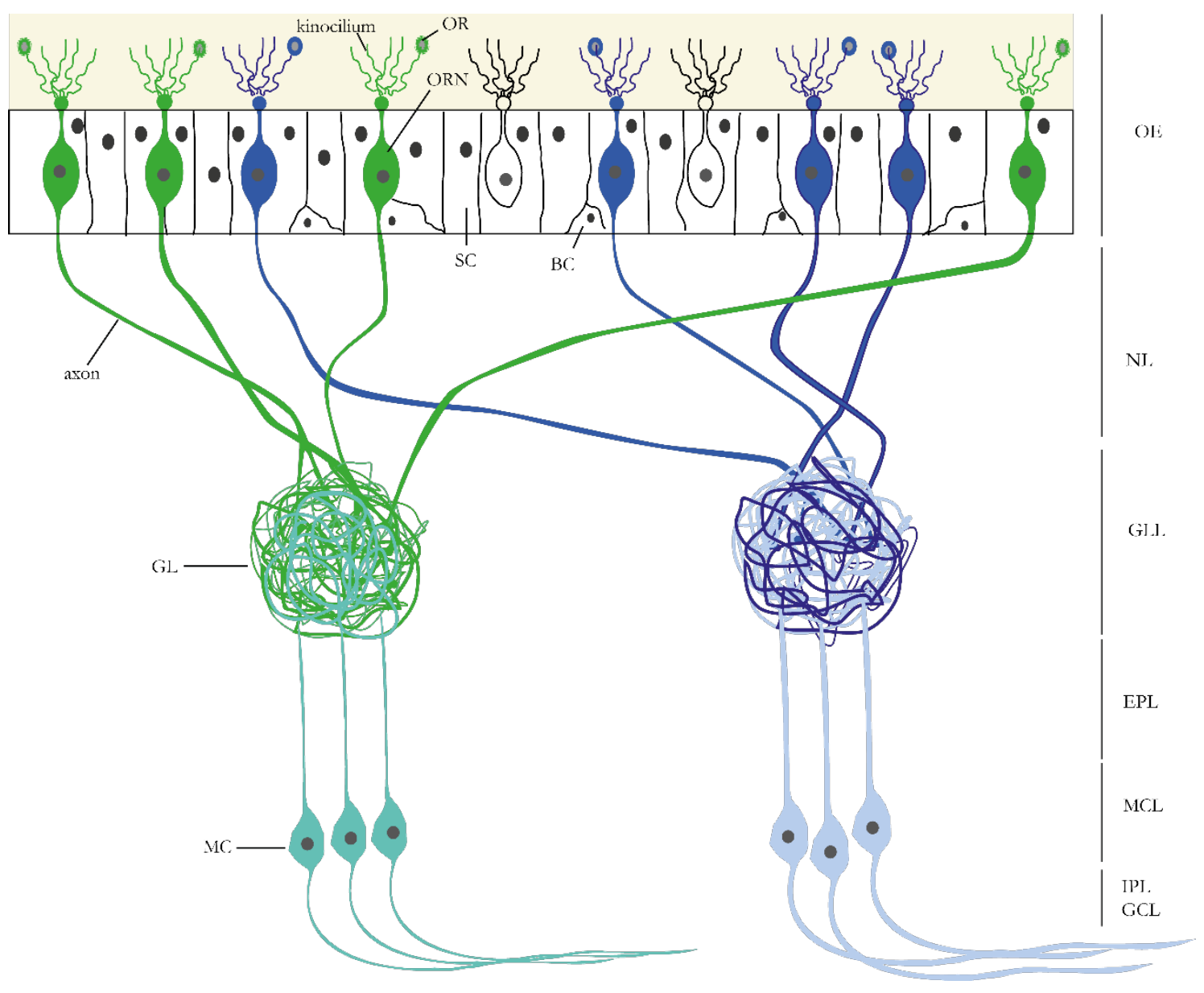

Figure 1 Schematic representation of the olfactory epithelium and olfactory bulb

The olfactory epithelium consists of a pseudostratified neuroepithelium harboring olfactory receptor neurons, sustentacular cells and basal cells. Each olfactory receptor neuron extends an apical dendrite ending in a dendritic knob in the nasal cavity. A variable number of kinocillia, embedded in a layer of mucus appear on the surface of the dendric knob. Sustentacular cells are column-shaped cells. Basal cells provide a stem cell pool of the epithelium for natural turnover and artificial damages to the epithelium. Each odorant receptor neuron sends a single axon to the olfactory bulb. For clarity purposes, only 8 odorant receptor neurons are represented in this sketch. The olfactory bulb has 6 discernable layers: the nerve layer (NL) which contains the axons of the olfactory receptor neurons, the glomerular layer (GLL) in which axon terminals of olfactory receptor neurons bearing the same olfactory receptor type project to the same glomerulus, the external plexiform layer (EPL), the mitral cell layer containing mitral and tufted cells (MCL), the internal plexiform layer (IPL) and finally the granule cell layer (GCL, granule cells not shown here). In larval Xenopus laevis ORN axons are branching and target on average 2-3 glomeruli and enter each glomerulus with 2 sub-branches (not shown in this simplified schematic). The glomerulus layer of the olfactory bulb contains only around 200 periglomerular cells in larval Xenopus laevis whereas in mammals periglomerular cells form a wall around the glomeruli (Chao et al. 1997). OE $=$ olfactory epithelium, $\mathrm{OR}=$ olfactory receptor, $\mathrm{ORN}=$ olfactory receptor neuron, $\mathrm{SC}=$ sustentacular cell, $\mathrm{BC}=$ basal cell, $\mathrm{OB}=$ olfactory bulb, $\mathrm{NL}=$ nerve layer, GLL = glomerular layer, $\mathrm{EPL}=$ external plexiform layer, $\mathrm{MCL}=$ mitral cell layer, $\mathrm{IPL}=$ internal plexiform layer, $\mathrm{GCL}=$ granule cell layer, $\mathrm{GL}=$ glomerulus, $\mathrm{MC}=$ mitral cell. 
The SCs are supporting cells in the OE which share communalities with both glial and epithelial cells. SCs in the OE of Xenopus laevis either bear microvilli and secretory granules or they bear kinocillia and possess no intracellular granular bodies (Hansen et al. 1998). These column-shaped cells are of eminent importance for the ORNs as they fulfill a battery of tasks. They were reported be involved in secretion (Hansen et al. 1998), endocytosis (Bannister and Dodson 1992), metabolism of toxicants (Thornton-Manning and Dahl 1997), physically insulating ORNs (Breipohl et al. 1974) and active phagocytosis of dead cells (Suzuki et al. 1996).They also regulate the extracellular ionic environment (Getchell 1986). Similarly to central glial cells, SCs exhibit intercellular calcium waves as well as intracellular calcium oscillations when being activated by P2Y-purinergic or muscarinergic receptor which may play an important role in damage-induced neurogenesis (Hegg et al. 2009). Other studies have shown the involvement of SCs in modulating the sensitivity of ORNs by purinergic (Hegg et al. 2003; Czesnik et al. 2006) and endocannabinoid signaling (Breunig et al. 2010).

Finally, globose basal cells provide the stem cell pool of the olfactory system. They can replace ORNs and SCs throughout life (Leung et al. 2007). Their activation seems to be mediated by purinergic signaling (Hassenklöver et al. 2009).

The OE of all vertebrates is covered by a mucus layer being produced from Bowman's glands, goblet cells or SCs (Getchell and Getchell 1992). The role of the mucus is to dissolve and therefore concentrate the volatile odorant molecules on the surface of the olfactory epithelium. Odorant binding proteins (OBPs) are often found in the mucus layer (Dal Monte et al. 1991; Tegoni et al. 2000). OBPs are a sub-class of lipocalins and provide the attitude to bind reversibly volatile chemicals, thereby facilitating the transport of hydrophobic odorant molecules from the gaseous phase to the aqueous mucus (Schiefner et al. 2015). However, the mucus layer only appears in the principal cavity of postmetamorphic Xenopus laevis and not in the larval principal cavity or adult middle cavity (Hansen et al. 1998).

Morphology of the OB. The axons of the ORNs form the first of six discernible layers in the olfactory bulb in larval Xenopus laevis (Manzini and Schild 2010). The axon terminals of the nerve layer synapse onto mitral and tufted cells in the glomerular layer. The axon terminals of ORNs in the MOE end in the main olfactory bulb (MOB) whereas the axons of ORNs in the VNO end in the accessory olfactory bulb (AOB) (Manzini and Schild 2010) suggesting a distinct spatial segregation of information of odorants and pheromones, respectively. The mitral and tufted cells are the second-order neurons of information processing and convey the signals to further brain centers. 
Non-chemical sensing properties of the olfactory system. As olfaction occurs in different environments, researchers have focused over the last years on the influence of different sensitivity properties of the olfactory system. Mechanosensitive ORNs responding to pressure were discovered in the nose of larval Xenopus laevis. Their axons ended in a specialized sub-region of the OB called the $\beta$-Glomerulus (Brinkmann and Schild 2016). Other ORNs in larval Xenopus laevis were thermo-sensitive and projected to another special region of the OB called the $\gamma$-Glomerulus (Kludt et al. 2015).

\subsubsection{Signal transduction and transformation in ORNs of larval Xenopus laevis}

The detection of odorants and pheromones begins with the binding of ligands to olfactory receptors (OR) that are expressed on the cilia or microvilli of ORNs. Adequate olfactory stimuli for larval Xenopus laevis are alcohols, aldehydes, ketones, bile acids, amines and amino acids (Gliem et al. 2009; Syed et al. 2013).

In vertebrates, 5 families of olfactory receptor genes are currently known: the "odorant receptor" OR-type, the vomeronasal receptors V1R and V2R, the trace-amine associated receptor TAAR and the formylpeptide receptor FPR. The receptor gene families seem to have developed at evolutionary different times and have conserved signaling pathways across species (Manzini and Korsching 2011). ORs belong to the family of G-protein coupled receptors. The expression of each receptor gene varies considerably among different species, that is between zero and several hundred receptor genes per cell (Manzini and Korsching 2011). Compared to other sensory systems a large portion of the genome is devoted to OR gene families varying between approximately 100 in fish to 1000 in mice (Malnic et al. 2004). However, there is a high frequency of so called pseudogenes, i.e. nonfunctional genes among the gene families. In humans over $50 \%$ of the olfactory receptor gene families were identified as pseudogenes (Menashe et al. 2006).

Moreover, a multitude of studies focusing on different animal models showed that each ORN expresses only one receptor type (Ressler et al. 1994a; Treloar et al. 1996; Mombaerts 2004). In addition to that, ORNs expressing the same receptor type would project their axons to the same glomerulus (Mombaerts 2006). These 2 conditions are considered the foundation of the combinatorial coding hypothesis which suggests that an odorant is coded by the activation of a specific spatial pattern of glomeruli, and each glomerulus representing one "epitope" of the molecular odor structure (Ressler et al. 1994b; Lledo et al. 2005). In addition, the temporal dynamics of glomerular activation are regarded as further information coding of the identity and concentration of the represented odor 
(Junek et al. 2010). In larval Xenopus laevis, however, there are doubts about this theory as a study showed 204 different response classes in 283 ORNs to amino acids (Manzini and Schild 2004a), suggesting more than one receptor per ORNs coding for amino acids. Moreover, the axon terminals of ORNs are widely branching and innervating mostly 2-3 different glomeruli simultaneously (Nezlin and Schild 2005). These 2 findings specific to Xenopus laevis come hardly in line with the one-receptor-one-glomerulus hypothesis.

In mammals, the MOE and VNO differ anatomically, morphologically and at molecular level. Ciliated ORNs of the MOE mainly express OR-type olfactory receptors that are coupled to an olfaction specific G-protein $G_{\text {olf }}$ (Kato and Touhara 2009). The binding of an odorant leads to the activation of $G_{\text {olf }}$ that in turn activates the adenylate cyclase III (ACIII). ACIII generates cyclic adenosine monophosphate (cAMP) which binds and opens the cyclic nucleotide-gated channel $(\mathrm{CNG})$. This channel is permissive for sodium $\left(\mathrm{Na}^{+}\right)$ and calcium $\left(\mathrm{Ca}^{2+}\right)$ ions. Influxing cations depolarize the membrane and therefore generate a receptor potential. The calcium ions themselves open a calcium-sensitive chloride $\left(\mathrm{Cl}^{-}\right)$ channel (CaCC) that leads to an efflux of chloride anions and therefore amplify the depolarization. In contrast, the VNO of mammals consists of microvillous ORNs that express V1R and V2R receptors and are coupled to $G_{i}$ and $G_{o}$, respectively, signaling via a phospholipase C (PLC) pathway. PLC generates inositol triphosphate (IP 3$)$, diacylglycerol (DAG) and arachidonic acid which activate a transient receptor potential channel 2 (TRPC2). TRPC2 is permeable for calcium and sodium cations (Kato and Touhara 2009). In larval Xenopus laevis, however, the MOE bears both ciliated and microvillous ORNs (see section 1.2.1). The olfactory signaling in the MOE of larval Xenopus laevis typically segregates into a medial and lateral stream (Gliem et al. 2013). Thereby, the medial stream consists of ciliated ORNs signaling via a cAMP-dependent pathway whereas the microvillous ORNs of the lateral stream do not (Manzini et al. 2002a). Amino acidsensitive ORNs of the lateral stream predominantly express V2R receptors and are coupled to $G_{i}$ or $G_{o}$ and use the phospholipase $C$ (PLC) and diacylglycerol (DAG) transduction pathway (Sansone et al. 2014). Interestingly, blocking $\mathrm{IP}_{3}$ and arachidonic acid did not affect the signaling in the ORNs suggesting that diacylglycerol is solely involved in activation of the putative transient receptor potential channel (TRPC) (Sansone et al. 2014). Phospholipase-C-independent transduction cascades of amino acid sensitive ORNs are only partially sensitive to forskolin (a cAMP-pathway activator (Frings and Lindemann 1991)). Taken together, these findings highlight at least 3 different signaling pathways of 
amino acid-sensitive ORNs: a cAMP-mediated pathway, the Phospholipase-CDiacylglycerol-pathway and a third unknown pathway (Sansone et al. 2014).

After a response was elicited by an odorant or pheromone the ORN needs to terminate the state of stimulation and return to a steady state. Only in this way it is possible to encode the temporal properties of an odor and enable subsequent stimulations. Various studies have been dedicated to investigate the molecular mechanisms underlying ORN recovery and adaptation to brief or sustained stimuli presentation. Essentially, at any level of the signal transduction cascade inhibitory steps can provide a tool to attenuate or terminate the signal propagation. As cAMP-mediated pathway was initially known as the generic signal transducation cascade in ORNs many studies focused on inhibiting factors of this specific pathway. Potential suppressors of stimulation status are protein kinase A (PKA) or Greceptor protein kinase 3 (GRK3) phosphorylating the OR and causing subsequent capping by $\beta$-arrestin (Dawson et al. 1993; Peppel et al. 1997; Mashukova et al. 2006), inhibition of ACIII by Ca-Calmodulin-dependent kinase II (Wei et al. 1998), hydrolysis of cAMP by phosphodiesterase enzymes (PDE) (Cygnar and Zhao 2009), desensitization of cyclic nucleotide gated channel by calcium-calmodulin (Liu et al. 1994) and sodium-dependent calcium extrusion that closes the calcium-dependent chloride channel (Reisert and Matthews 1998). Other studies considered ion exchangers as being important for shortterm recovery compared to long-term recovery after stimulation (Pyrski et al. 2007). Which of the mechanisms contribute mostly to ORN recovery remains a controversial and elusive topic.

\subsection{Spontaneous activity and sensitivity of ORNs}

\subsubsection{Spontaneous activity in ORNs}

Neuronal cells like ORNs can generate action potentials (APs) in the absence of stimuli. Such activity is often referred to as spontaneous activity or spontaneous firing rate (SFR). The origin and the function of such an activity have been the focus of many studies in the past 3 decades. Some authors classify spontaneous activity simply as intrinsic noise of receptor neurons contributing to signal-to-noise ratio in neural networks (Savigner et al. 2009). However, many studies have reported several useful functions of spontaneous activity in different neuronal networks of the central and peripheral nervous system. Firstly, spontaneous activity can widen the cellular coding dynamic range in the sense that not only the increase of firing rate but also the reduction of tonic activity can code for the presence 
or absence of a stimulus. Such inhibitory coding patterns are common in shaping an input signal via lateral inhibition (Olsen and Wilson 2008). In the peripheral olfactory system inhibition of tonic activity, i.e. inhibitory responses, were reported for several aquatic species (Kang and Caprio 1997; Vogler and Schild 1999). Secondly, the spontaneous activity of neurons in developing organisms seems to play an outstanding role in axonal targeting and synaptic formation (Spitzer 2006). Several studies of different neuronal systems like the motor (Hanson and Landmesser 2004), visual (Katz and Shatz 1996), auditory (Tritsch et al. 2007) and olfactory system (Yu et al. 2004) have shown that spontaneous activity of primary sensory or motor neurons are required to establish and maintain the synaptic formation.

Nonetheless, the origin of spontaneous activity in neurons is still unknown. Speculations about possible sources of spontaneous activity in the peripheral olfactory system led to investigations of nearly all molecules involved in the signal transduction pathway. Among them were intracellular cAMP levels (Nakashima A et al. 2013), the hyperpolarisationactivated cyclic nucleotide-gated cation channel (HCN) (Nakashima N et al. 2013), CaCCs (Reisert 2010; Pietra et al. 2016) and molecules not directly involved in transduction pathways like the voltage-gated sodium channel $\mathrm{Na}_{\mathrm{v} 1.5}$ (Frenz et al. 2014). However, most researchers focused on olfactory receptors as the main source of spontaneous activity (Serizawa et al. 2006; Reisert 2010; Connelly et al. 2013). A common hypothesis states that OR-induced stereotypic activity determines the intracellular cAMP-level and subsequent activation of protein kinase $\mathrm{A}$ which in turn regulates the transcription level of molecules like Neuropilin-1 and Kirrel2/Kirrel3. The latter are important for the correct axonal guidance and refinement of glomerular formation in developing vertebrates (Serizawa et al. 2006; Nakashima A et al. 2013). Which of the putative sources mainly contributes to spontaneous activation of ORNs remains, however, elusive.

Many authors reported spontaneous firing rates in ORNs mostly below 1 to 4 APs per second (van Drongelen et al. 1978; Getchell 1986; Rospars et al. 1994; Duchamp-Viret et al. 2000; Connelly et al. 2013; Rospars et al. 2013). Thereby, interspecies comparison showed differences in the basal activity; for instance, the reported spontaneous firing rates in catfish Ictalurus punctatus of approximately 4.7 APs per second were higher than those observed in terrestrial vertebrates. Conversely, the inhibitory responses in that species were more frequently observed than excitatory responses (Kang and Caprio 1995). In larval Xenopus laevis a previous study by colleagues compared SFRs of ORNs in 2 methodological preparations. Thereby, these researchers found that the averaged SFRs of larval Xenopus laevis ORNs were on average 3 APs per second for isolated cells and 2-13 APs per second 
in acute slice preparations, highlighting the importance of a methodical approach to record spontaneous and stimulus-induced signals in electrophysiology (Manzini et al. 2002b).

Regarding the stochastic character of spontaneous AP generation in ORNs a few authors reported empirical and theoretical descriptions (Rospars et al. 2013). Assuming a nearly constant rate of AP generation in the absence of stimuli, i.e. stationarity in mean and variance of the spontaneous firing rate, and that mean and variance of the rate being at the same order of magnitude then the most appropriate theoretical distribution to describe the spontaneous activity in ORNs would be the Poisson distribution. However, different authors reported contradictory results on the Poissonian character of SFRs in ORNs with two authors being opposed to the Poissonian character (Getchell 1986; Rospars et al. 1994) versus one author in favor of the theory (van Drongelen et al. 1978).

\subsubsection{Sensitivity of ORNs}

The sensitivity of ORNs can be described in quality and quantity. The olfactory system is able to detect a large number of water-soluble or air-borne odorants through olfactory receptors. Thereby, an odor typically consists of several different chemical molecules. Olfactory receptors seem to be designed to bind specific "targets" of odor molecules, for instance the functional group in alcohols or amines (Gliem et al. 2009). Activation of a set of ORNs binding specific "epitopes" of the odorant mix leads to activation of epitope maps at the olfactory bulb level (Ressler et al. 1994b). As one olfactory receptor can bind several odor molecules and each molecule can be detected by different receptors this yields in a broad tuning of ORNs such that virtually all possible combinations of odorant mixtures can be represented by activation of a specific subset of ORNs (Firestein 2001). In aquatic animals, amino acids were identified as appropriate stimuli for olfactory receptors (Caprio 1978), and therefore typically used in several studies of our institute (Manzini and Schild 2003; Manzini et al. 2007; Gliem et al. 2013). Accordingly, amino acids are used as stimuli in the scope of this thesis as well.

Many authors have dedicated multiple studies to address the question of ORN sensitivity in a quantitative manner. They found that the EC-50 value, i.e. the concentration value activating half of the neuronal population, of dose-response curves were similar in most vertebrates in different species and under different experimental conditions. The reported EC-50 values ranged from $3 \mu \mathrm{M}$ to $90 \mu \mathrm{M}$ in tiger salamander (Firestein et al. 1993), 4.4 $\mu \mathrm{M}$ to $104 \mu \mathrm{M}$ in mice (Grosmaitre et al. 2006) and to $1 \mu \mathrm{M}$ to $10 \mu \mathrm{M}$ in rats (DuchampViret et al. 1999). A fraction of the tested ORNs responded even to concentrations in the 
nanomolar ranges (Duchamp-Viret et al. 1999; Grosmaitre et al. 2006). For larval Xenopus laevis there has been no complete quantitative description of ORN sensitivity existing so far. A previous study described concentration threshold values of $200 \mathrm{nM}$ to $100 \mu \mathrm{M}$ using amino acids as stimuli (Breunig et al. 2010). Thereby the author focused on the influence of endocannabinoids on ORN sensitivity and showed that the threshold for stimulus detection for all ORNs was increased by antagonizing the endocannabinoid pathway. Nonetheless, the wide range of stimulus threshold concentrations is astonishing as the ORNs seem to differ in their (threshold) sensitivities over several orders of magnitude.

\subsection{Scope of the thesis}

The scope of this thesis is to quantitatively investigate ORN activity in presence and absence of an adequate stimulus and thereby understand basic principles underlying olfactory coding at the level of receptor neurons.

Larval Xenopus laevis is a developing vertebrate which, unlike mammalian embryos, is living outside the maternal body. Therefore, it provides the unique opportunity to access the sensory systems easily and investigate neuronal activity under experimental conditions. In mammalian embryos, it has been demonstrated that spontaneous activity of ORNs was important for the development and refinement of olfactory system. The level of activity and associated intracellular cAMP levels were indispensable for anterior-posterior targeting of receptor neurons' axonal terminals (Serizawa et al. 2006; Nakashima et al. 2013a). For larval Xenopus laevis a quantitative analysis of ORNs' spontaneous activity is still lacking. What is the range of spontaneous activity in ORNs? Is spontaneous activity in ORNs a random process, and if so, which stochastical model may be appropriate to describe spontaneous activity in ORNs?

Although many studies were dedicated to understand response profiles and intracellular signaling pathways in larval Xenopus laevis ORNs (Vogler and Schild 1999; Manzini and Schild 2003; Manzini and Schild 2004a; Manzini et al. 2007; Gliem et al. 2013), there is still no complete quantitative description of stimulus-induced changes in cellular activity of ORNs existing. Detailed stimulus-induced dose response curves were reported in many other species, including the tiger salamander (Firestein et al. 1993), mice (Grosmaitre et al. 2006) and rats (Duchamp-Viret et al. 1999). In larval Xenopus leavis it is therefore important to elucidade following questions: which stimulus concentrations elicit a response in ORNs? What is the saturation concentration for amino acids as stimuli? What is the concentration activating half of the ORN population, i.e. the EC-50 concentration? How does spontaneous activity in ORNs fluctuate following stimulus application, i.e. how is the coding of stimulus identity and concentration performed at the ORN level? Are AP frequency and number of APs sufficient to encode information of odor concentration? 
On the one hand, patch-clamp experiments have shown that it can be hard to distinguish stimulus-induced activity from spontaneous activity at threshold concentrations. On the other hand, some authors suggested that the binding of single odor molecules induce activity in ORNs (Menini et al. 1995). Is there a way to distinguish stimulus induced activity from spontaneous activity at threshold concentrations?

In order to answer these questions appropriately I decided to combine the techniques of intracellular $\mathrm{Ca}^{2+}$-imaging with the benefits of the cell-attached patch-clamp technique. By first identifying appropriate amino acid-sensitive ORNs and then patching the cell it was possible to record both spontaneous and stimulus-induced activity of the index cell. 


\section{$2 \quad$ Materials and methods}

\subsection{The experimental animal: larval Xenopus laevis}

\subsubsection{General description}

The African clawed frog Xenopus laevis belongs to the family of pipidae and to the anurian order of the amphibian class (Tinsley et al. 2009). Its native habitat is central and southern Africa but the species of Xenopus laevis have been introduced to many countries outside of Africa as well, mainly for the purposes of scientific research and pregnancy testing (Shapiro und Zwarenstein 1934; Nieuwkoop und Faber 1994). The adult animals have a semiterrestial and semi-aquatic lifestyle, inhabiting preferentially warm, stagnant ponds that are covered with green algae. Xenopus laevis adults possess claws to climb and shred food (Maddin et al. 2009). These animals are scavengers and will eat mostly living or dead arthropods and other pieces of organic waste (Avila and Frye 1977).

Like most other amphibians, the life cycle of the African clawed frog includes metamorphosis (Nieuwkoop and Faber 1994). The fertilized eggs of the female adult frog develop into free swimming larvae which keep to a fully aquatic lifestyle (Manzini and Schild 2010). The developmental stages of larval Xenopus laevis have been described in detail by Nieuwkoop and Faber (1994). Xenopus laevis proves to be a valuable laboratory animal as its vertebrate embryological development occurs outside the maternal body. Furthermore, during premetamorphic stages, the tadpole's brain is not encapsulated by cartilage or bony plates.

\subsubsection{The olfactory system of Xenopus laevis}

The tadpoles of Xenopus laevis exhibit 2 distinct olfactory organs, the principal cavity and the vomeronasal organ (VNO). A third organ, the middle cavity, appears during metamorphosis. While ORNs of the VNO and middle cavity detect water-borne odorants throughout the life of the animal, those of the principal cavity only does so in larval stages; in adult animals, ORNs of the principal cavity detect air-borne odorants (Hansen et al. 1998). The cells in these cavities bear either microvilli or kinocillia depending on the cell type, the location and the developmental stages. ORNs in the VNO always bear microvilli whereas the SCs are ciliated throughout life. In larval principal cavity the ORNs are of 2 
types: ciliated and microvilliar, while the SCs are either ciliated or secretory with small granules. After metamorphosis the kinocillia of the ORNs are longer compared their size during the larval stages, and microvillar ORNs are absent in the principal cavity. SCs of the adult principal cavity have electron-dense large granules. The new evolving middle cavity contains a cell architecture comparable to that of the larval principal cavity (Hansen et al. 1998). The cellular protuberates, kinocillia and microvilli, are important features as they are associated with different signaling mechanisms. Xenopus laevis provides a unique insight into the transition of aquatic to terrestrial animals as it is interposed from an evolutionary perspective between fish and fully terrestrial vertebrates. This interposition is reflected at cellular and molecular level. The MOE of larval Xenopus laevis resembles the single sensory surface of teleost fishes (Hamdani et al. 2008) in that it contains both microvillar and ciliated ORNs. The microvillous ORNs express V2R genes (Syed et al. 2013) signal via a PLC-mediated pathway (Sansone et al. 2014). In fish and larval Xenopus laevis V2Rs are sensitive to amino acids whereas in higher vertebrates they are activated by pheromones. In contrast, the VNO of larval Xenopus laevis is reminiscent of rodent VNO (Munger 2009).

\subsection{Slice preparation, staining protocol and solutions}

\subsubsection{Slice preparation}

Xenopus laevis tadpoles of developmental stages 51 to 54 (Nieuwkoop and Faber 1994) were used for all experiments. The animals were chilled in ice water for at least 2 minutes. Subsequently, the motionless and cold-anesthetized tadpoles were fixed on a preparation plate and killed by transection at brain level. All procedures for animal handling and tissue dissections were carried out according to the guidelines of the Göttingen University Committee for Ethics in animal experimentation.

A block of tissue containing the olfactory epithelia, both olfactory nerves and the olfactory bulb was cut out from the tadpole and glued onto a plate that was placed into the vibroslicer (VT 1200S; Leica Microsystems GmbH, Wetzlar, Germany). Brain slices of a thickness of $200 \mu \mathrm{m}$ were cut from the tissue block. The slices were then transferred into a prepared dish and perfused in Ringer's solution (Figure 2). 


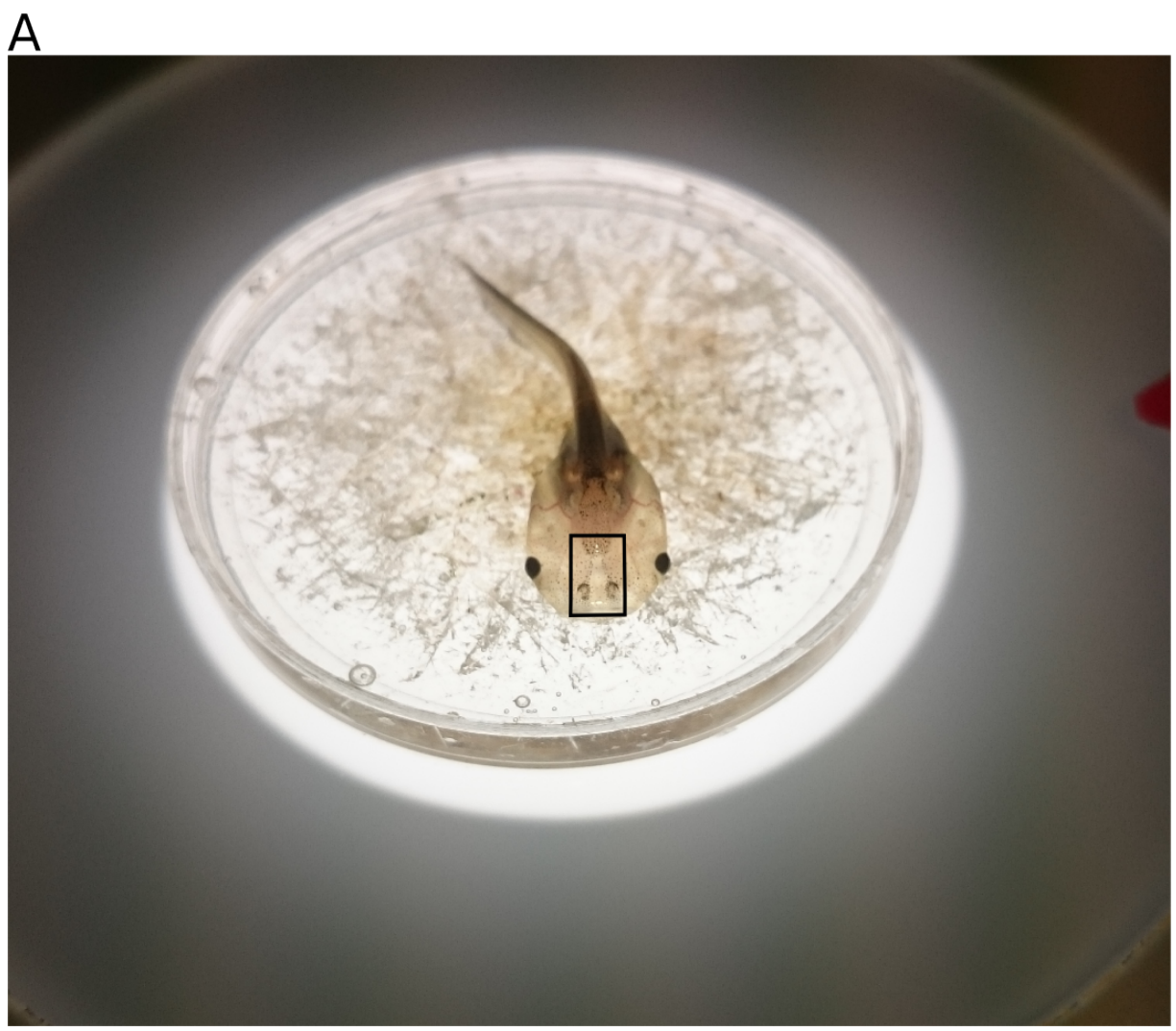

B

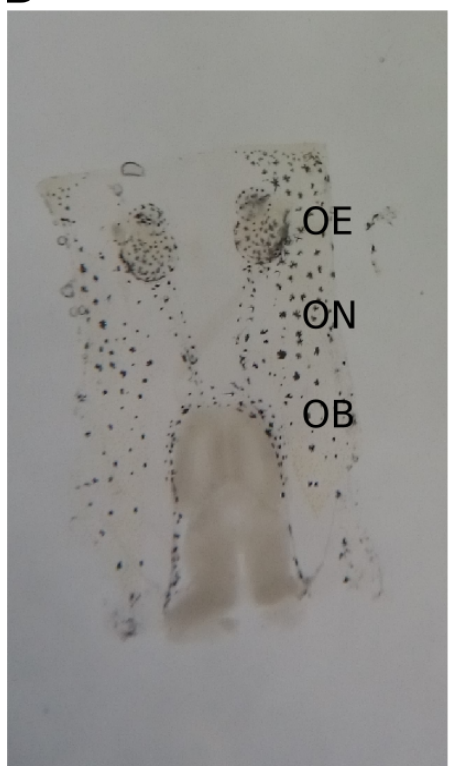

C

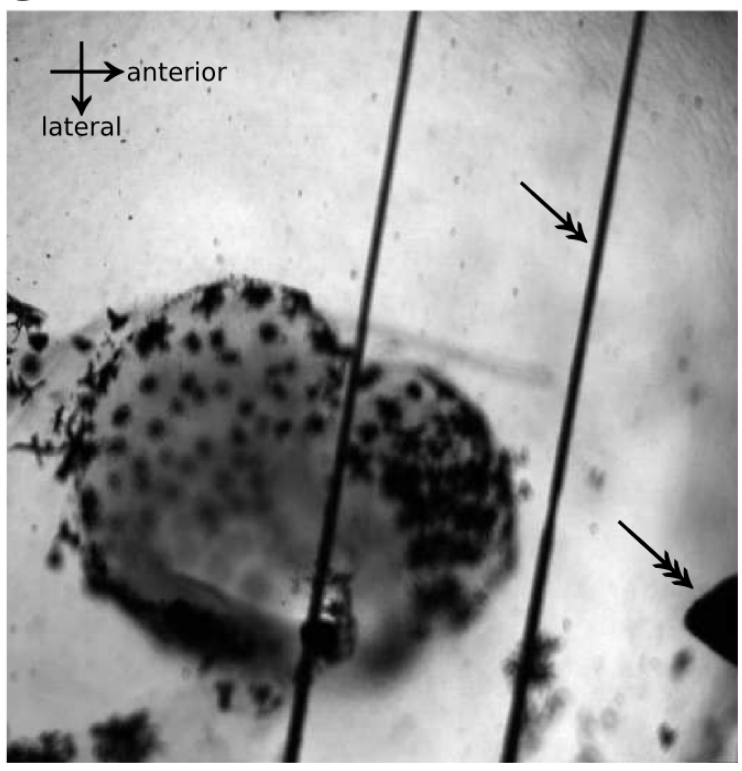

Figure 2 Slice preparation of larval Xenopus laevis

A| Xenopus laevis larva on a preparation plate after being anesthetized in ice water. The black rectangle indicates the area that was dissected in order to preserve a block of tissue containing the olfactory epithelium, the olfactory nerves and the olfactory bulb. B $\mid$ The tissue block was cut into ca. $200 \mu \mathrm{m}$-thick slices. C| The prepared slices were bathed in Ringer's solution and kept in place with horizontally arranged strings (arrow with double head). This way, it was possible to apply the solution close to the olfactory epithelium (arrow with triple head indicates the tip of the stimulus application funnel). 10x magnification under the objective of an upright microscope. $\mathrm{OE}=$ olfactory epithelium, $\mathrm{ON}=$ olfactory nerve, $\mathrm{OB}=$ olfactory bulb. 


\subsubsection{Staining protocol}

In order to stain ORNs, the fluorescent calcium dye Fluo-8-AM (Molecular probes, Leiden, Netherlands) was chosen. Thereby $50 \mu \mathrm{g}$ Fluo-8-AM was dissolved in Ringer's solution, dimethyl sulfoxide (DMSO; Sigma Aldrich, St. Louis, USA) and Pluronic acid F-127 (Simga Aldrich, St. Louis, USA). In a second step, the dissolved solution was diluted in Ringer's solution, so that the final concentrations of DMSO and Pluronic acid F-127 did not exceed $0.5 \%$ and $1 \%$, respectively. There is evidence showing that ORNs of larval Xenopus laevis express multidrug resistance transporters with a wide substrate spectrum including calciumindicator dyes (Manzini and Schild 2004b). Consequently, the multidrug receptor protein (MDR-P)-inhibitor MK-571 (Enzo Life Sciences, Lörrach, Germany) was applied to the staining solution in order to prevent a precocious bleaching of the tissue.

$200 \mu \mathrm{L}$ of the staining solution were added to the tissue slice, and the incubation lasted 30 min at room temperature. Next, the tissue was rinsed and held in Ringer's solution and the recording dish was thereafter placed under the microscope with a laser scanning unit for further electrophysiological measurements. Before the onset of each experiment, the tissue was super-perfused with Ringer's solution for 10 min. A continuous flow of Ringer's solution was maintained alike.

The method and solutions described have been commonly used in our institute and were previously reported in other studies extensively (Manzini et al. 2002b; Manzini et al. 2007; Gliem et al. 2013). This description reflects the specific set up of my own experiments.

\subsubsection{Applied solutions}

Aliquots of single amino acid solutions at $100 \mu \mathrm{M}$ were prepared in advance and kept at 4 ${ }^{\circ} \mathrm{C}$ in the refrigerator. The amino acid mixtures (Table 1) were then combined directly before the start of the electrophysiological experiments.

During the incubation time the aliquots were gently warmed to room temperature. The final temperature of the amino acid solutions and the Ringer's solution (Table 2) ranged between 18 and $23^{\circ} \mathrm{C}$. 
Table 1 List of amino acid subgroups and single amino acids

Amino acids subgroup

Basic amino acids

Long chain amino acids

Short chain amino acids

Aromatic amino acids

\section{Single amino acids}

L-arginine, L-histidine, L-lysine

L-methionine, L-leucine

L-alanine

L-tryptophan, L-phenylalanine

The Ringer's solution used for all experiments contained following components and concentrations:

Table 2 Composition of the Ringer's solution

Ringer's solution components

$\mathrm{NaCl}$

$\mathrm{KCl}$

$\mathrm{CaCl}_{2}$

$\mathrm{MgCl}_{2}$

Na-Pyruvate

Glucose

HEPES*

$\mathrm{pH}$

Osmolarity
Concentration, pH, Osmolarity

$100 \mathrm{mM}$

$2 \mathrm{mM}$

$1.5 \mathrm{mM}$

$0.5 \mathrm{mM}$

$5 \mathrm{mM}$

$5 \mathrm{mM}$

$10 \mathrm{mM}$

7.8

$231 \mathrm{mOsmol} / \mathrm{L}$

*(2-hydroxyethyl)-1-piperazineethanesulfonic acid

Aliquots of the staining solution containing Ringer's solution, DMSO (0.5\%), Pluronic acid F-127 (1\%), Fluo-8-AM and the MDR-P MK-57 were kept at $-18{ }^{\circ} \mathrm{C}$ and subsequently thawed at room temperature before slice staining.

All chemicals used were purchased from Merck (Darmstadt, Germany) or Sigma (St Louis, MO, USA) if not otherwise noted. 


\subsection{Electrophysiology}

\subsubsection{Calcium imaging}

In order to detect responses to stimulus applications and thereby identify amino acidsensitive ORNs, I decided to use calcium imaging. Changes in intracellular calcium concentrations - which indicate a cell's response - can be visualized using a confocal laserscanning microscope (LSM) which applies laser light that excites fluorescent intracellular calcium dyes. The fluorescence signals and depolarization-induced changes in intracellular calcium concentration were monitored using the Zeiss ZEN-Software. Excitation of the fluorescent dye was achieved by an Argon laser sending monochromatic laser light at a wavelength of $\lambda=488 \mathrm{~nm}$ and the emission spectrum covered the wavelength range from $493 \mathrm{~nm}$ to $622 \mathrm{~nm}$. The framing interval was $963 \mathrm{~ms}$ and 60 frames were taken as time series. A calcium wave was characterized as a response if the ORN showed a distinct increase of fluorescence signal after the application of a $100 \mu \mathrm{M}$-concentrated mixture of all amino acids listed in Table 1. The application of the stimulus solution was performed by an electrical pipette after a defined delay time ( $5 \mathrm{~s}$ in all experiments). Before and after each application of the stimulus $1 \mathrm{ml}$ of Ringer's solution was applied as negative control.

For this purpose, the tissue sample was placed directly under the objective of the microscope. Simultaneously a continuous flow of Ringer's solution through the recording chamber was maintained by means of a storage syringe system applying Ringer's solution by gravity forces. A drug application system using a needle as funnel was placed in direct proximity of the OE, allowing the application of stimulating solutions without interrupting the continuous flow of Ringer's solution (Figure 3). The dilution of stimulus concentration at the end of the funnel was assumed to be less than 1\% (Manzini and Schild 2003). In this way it was possible to identify amino acid-sensitive ORNs which were mostly located in lateral area of $\mathrm{OE}$, in consistence with a previous study from our research group (Gliem et al. 2013). After ORNs showed a response, a second mixture containing subgroups of amino acids at $100 \mu \mathrm{M}$, i.e. basic, long-chained, short-chained and aromatic amino acids, was applied to the OE. In a third step a single amino acid solution $(100 \mu \mathrm{M})$ from a subgroup was applied as stimulus if a receptor cell had shown a response to the corresponding subgroup afore. Before and after each stimulus $1 \mathrm{ml}$ of Ringer's solution was applied as negative control. An ORN which was identified as sensitive for a certain amino acid was then labeled for further patch-clamp experiments. The tissue slice was used for a single experiment, i.e. only 1 ORN of the tissue was stimulated for 1 amino acid. 


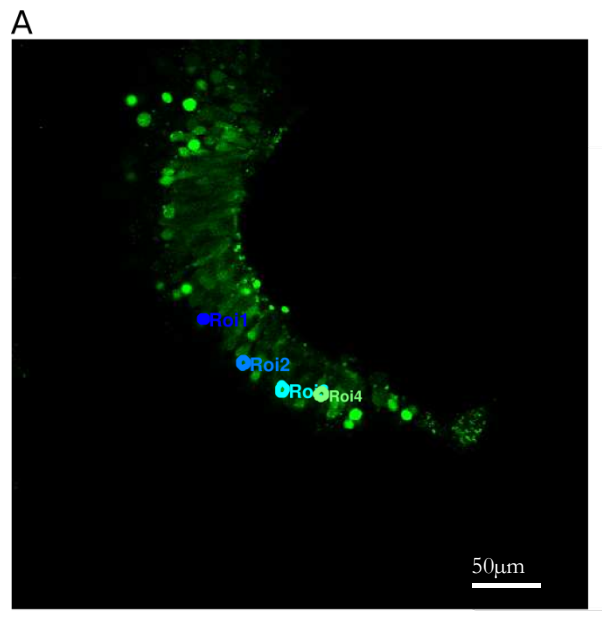

B

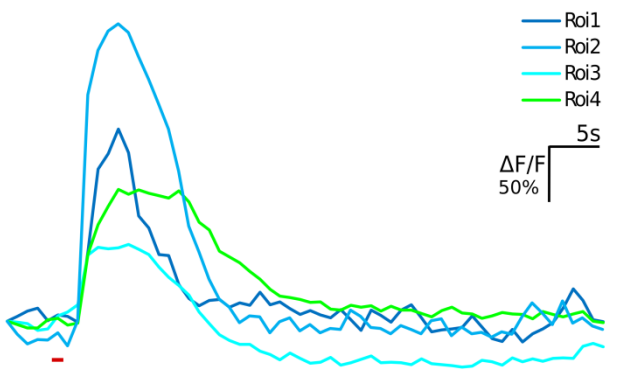

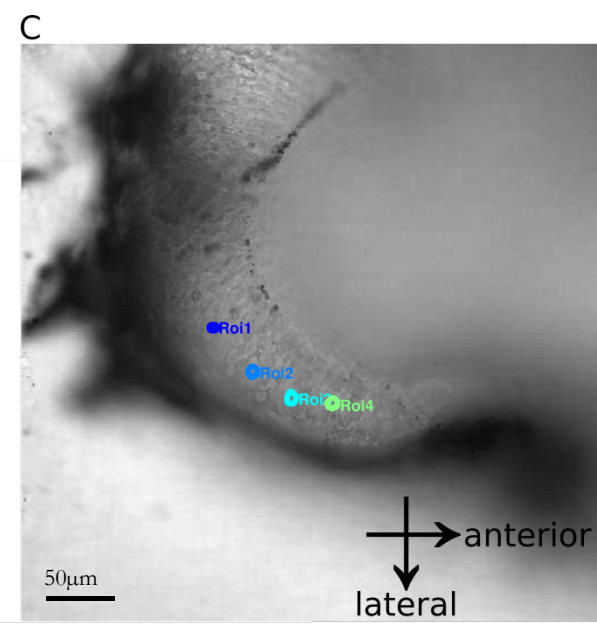

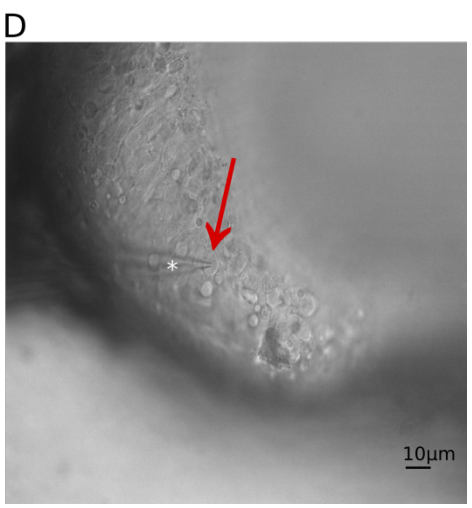

Figure 3 Calcium imaging and single-cell patching of ORNs

A| The intracellular Ca-dye Fluo-8-AM excited by the monochromatic laser light results in fluorescence signal. Selection of region of interests (ROI) for identification of ORNs responsive the applied stimulus (fluorescence signal in $\mathbf{A}$ and transmission channel in $\mathbf{C}$ ). Depolarization of the neuron increases the intracellular calcium concentration leading to binding calcium to the calciumsensitive dye. B $\mid$ The resulting increase in fluorescence signal indicates a response. Short red line represents stimulus application. D| Approaching the responsive cell (red arrow) with the micropipette (white star).

\subsubsection{Voltage clamp in the cell-attached mode}

The labeled ORN was localized after switching to the video recording mode in the LSMmicroscope on a second monitor.

For the patch clamp experiments patch microelectrodes with a tip diameter of $1-2 \mu \mathrm{m}$ and an electrode resistance of $\mathrm{R}=5-7 \mathrm{M} \Omega$ were used. These microelectrodes were pulled from borosilicate glass capillaries with $1.8 \mathrm{~mm}$ outer diameter (Hilgenberg, Malsfeld, Germany) using a two-stage pipette puller (PC-10, Narishige, Japan). The pipettes were filled with Ringer's solution and fixed in the electrode holder that was connected to a micromanipulator and the patch-clamp amplifier (EPC8, HEKA, Lambrecht, Germany). 
The patch-clamp experiments were carried out in the cell-attached mode. After forming a seal resistance in the $G \Omega$ range, the holding potential was set to $U_{\text {hold }}=-70 \mathrm{mV}$ and fast transient cancellation was performed.

The spontaneous activity of the labeled ORN was then observed for several minutes and after applying Ringer's solution as negative control, $1 \mathrm{ml}$ of a single amino acid solution at $100 \mu \mathrm{M}$ was released into the drug funnel. If the ORN repeatedly responded to stimulus application - and showed no response to Ringer's solution - a concentration ramp of that amino acid was generated in order to stimulate the cell at different concentrations while continuously recording its electrical response in the cell-attached mode. The stimulus paradigm of concentration increments was as follows: $10 \mathrm{nM}-100 \mathrm{nM}-1 \mu \mathrm{M}-10 \mu \mathrm{M}-$ $100 \mu \mathrm{M}$ (for some ORNs $25 \mu \mathrm{M}$ and $50 \mu \mathrm{M}$ concentrations were added as stimuli between $10 \mu \mathrm{M}$ and $100 \mu \mathrm{M}$ ), i.e. a ramp covering a 5-log-scale concentration range of stimulus.

Hence, the clamped ORN was continuously super-perfused with Ringer's solution for at least $5 \mathrm{~min}$ before the start of experiments, so that all amino acid remnants in the drug funnel were washed out and that the ORN recovered its spontaneous activity. Before and after each amino acid application $1 \mathrm{ml}$ of Ringer's solution was applied as control without interrupting the recording of ORN activity.

Each experiment started with the lowest concentration, i.e. $10 \mathrm{nM}$, and the following amino acid applications were characterized by increasing concentrations. The minimum interval between 2 applications was $1 \mathrm{~min}$; the minimum recording time for each cell was therefore $300 \mathrm{~s}$. Furthermore, after each stimulus application the drug funnel and its tube were rinsed with $1 \mathrm{ml}$ of Ringer's solution in addition to the constant flow from the storage syringe system. Thus, it was possible to wash out possible amino acid solution remnants in the tube as well as detect artificial responses of ORNs due to mechanic or thermal fluctuations.

The data acquisition was performed with the "patchmaster" software (HEKA Electronics). The evaluation and analysis of the ORN spiking activities was achieved offline by using Matlab (MathWorks, Natick, USA), GraphPad Prism 8 (GraphPad Software, San Diego, USA) and Microsoft Excel (Microsoft, Washington, USA). 


\section{Results}

\subsection{Spontaneous activity and sensitivity in ORNs}

\subsubsection{The spontaneous firing rates}

In each slice, one single receptor neuron was selected for voltage clamp in the cell-attached mode (see section 2.3). As a first step the spontaneous firing rates (SFRs) of ORNs were recorded. Thereby, a considerable variance in the SFRs across different ORNs was observed (Figure 4). The spontaneous firing activity of 46 ORNs were recorded in total and varied from 0.24 to 5.5 spikes per second, i.e. on average there was an approximately 20-fold higher firing rate in the fastest ORN compared to the slowest ORN (Table 3). The spontaneous firing rates were calculated as an average of ORN activity recorded over $30 \mathrm{~s}$ before each stimulus application.

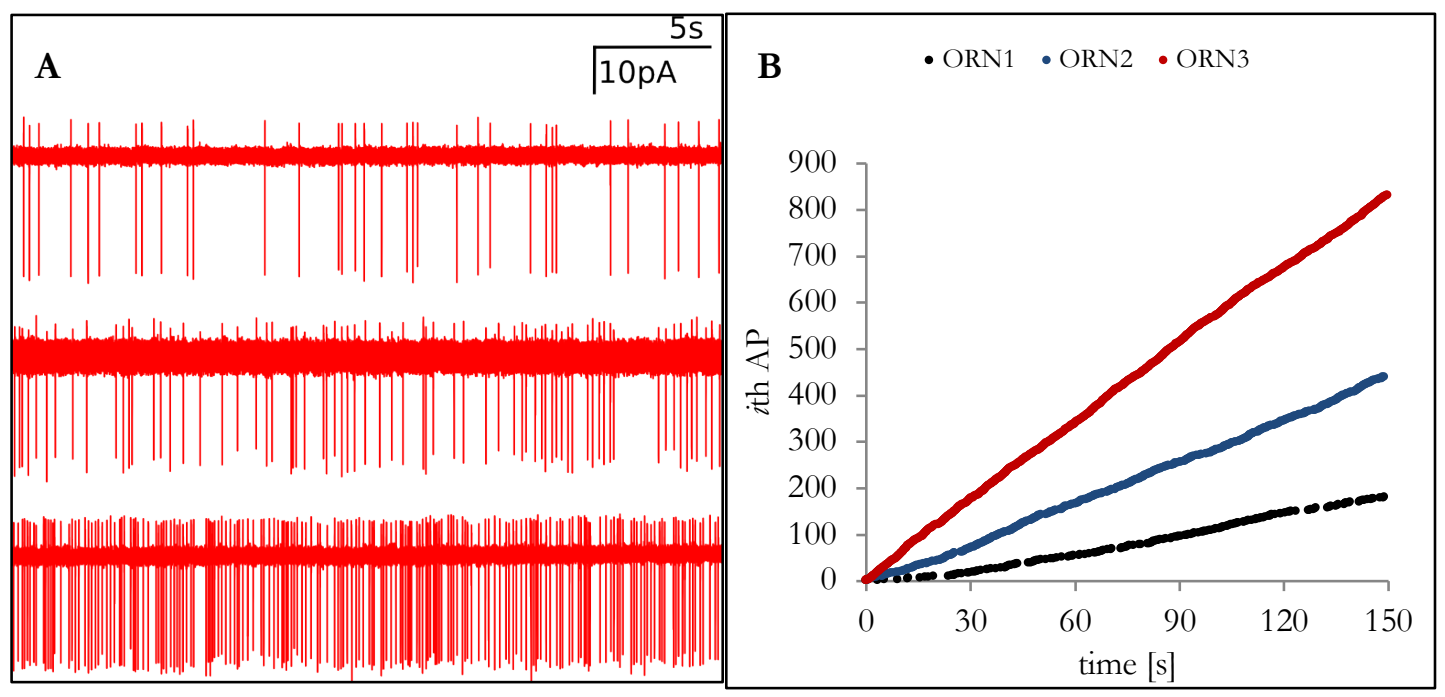

Figure 4 Spontaneous firing rates of 3 ORNs

A $\mid$ Raw spiking trains of 3 different ORNs (30 s-long recordings are displayed) in voltage clamp cell-attached mode. The upper ORN (ORN1 in B) is thereby a slowly firing ORN (averaged $\left.\mathrm{SFR}=1.03 \mathrm{~s}^{-1}\right)$, the lower cell (ORN3 in $\mathrm{B}$, mean $\mathrm{SFR}=5.5 \mathrm{~s}^{-1}$ ) is representative for fast firing ORNs. The ORN in-between (ORN2 in $\mathrm{B}, \mathrm{SFR}=2.76 \mathrm{~s}^{-1}$ ) takes an intermediate position. $\mathbf{B} \mid$ The number of action potentials (y-axis) is plotted against a recording time of $150 \mathrm{~s}$. All 3 ORNs represent different spontaneous activities in steady state. Notably, the data points of all 3 ORNs represent 5 recordings of $30 \mathrm{~s}$ before each stimulus application. Nonetheless, the slopes of the SFR curves are nearly constant. SFR $=$ spontaneous firing rate, $\mathrm{ORN}=$ odorant receptor neuron. 
The overall median SFR of 46 ORNs is 1.64 APs per second (Figure 5). The distribution of averaged SFRs is non-symmetrical and shows a positive skewness (empirical skewness $g=$ 1.03; median < mean (1.92 APs/s)). Accordingly, the distribution was statistically not Gaussian (Kolmogorov-Smirnov test (KST), $\mathrm{p}=0.048, \alpha=5 \%$ ).

The averaged SFRs, however, reduce the cellular activity to a single value. Therefore, it was necessary to investigate the spiking patterns of single cells. Did a single ORN fire spikes in the spectrum of the averaged SFRs? If spike generation was a random process for each single receptor neuron then how big would be the variance in the temporal spiking pattern? And, which stochastical process might be appropriate to model the spontaneous firing rates? As already seen for 3 different ORNs in Figure 4 the SFRs were different across neurons but nearly constant over time for a specific ORN. It was notable that the SFRs of each ORN did not change despite application of high stimulus concentrations, i.e. ORNs had entirely recovered to their baseline activity $30 \mathrm{~s}$ before the each stimulus application. As the SFRs were nearly constant for each ORN it was reasonable to test if AP generation in the steady state was a Poissonian process (Figure 6). Accordingly, the averaged SFRs of ORNs were used as an estimate of the event rate $\lambda$. In a second step the event rate $\lambda$ was adjusted such that $D=\sup \left|F_{0}(k)-S_{n}(k)\right|$ was minimum, where sup is the supremum function, $F_{0}(k)=e^{-\lambda} \sum_{i=0}^{k} \frac{\lambda^{i}}{i !}$ is the Poisson cumulative distribution function and $S_{n}(k)$ is the empirical cumulative distribution function representing the cumulative fraction of $\mathrm{n}$ observations which are less than or equal to $\mathrm{k}$.

For this purpose a sample of 36 ORNs (sensitive to alanine $(n=14)$, arginine $(n=11)$ and methionine $(n=11)$ ) was selected. For each ORN the SFR was determined for 60 s of recording time and number of events (i.e. APs or k) was counted for every second (i.e. bin width, $\mathrm{n}=60$ observations). In 35/36 ORNs the SFR was a good estimation of event rate $\lambda$ so that the observed events were conform to the theoretical Poisson model (Kolmogorov-Smirnov test, $\alpha=1 \%$ ). However, when testing more strictly (i.e. at $\alpha=5 \%$ ) 5 ORNs had a distinctly different distribution than expected in a Poisson process. The reason for this discrepancy lay in the variance of spike generation in those neurons. 


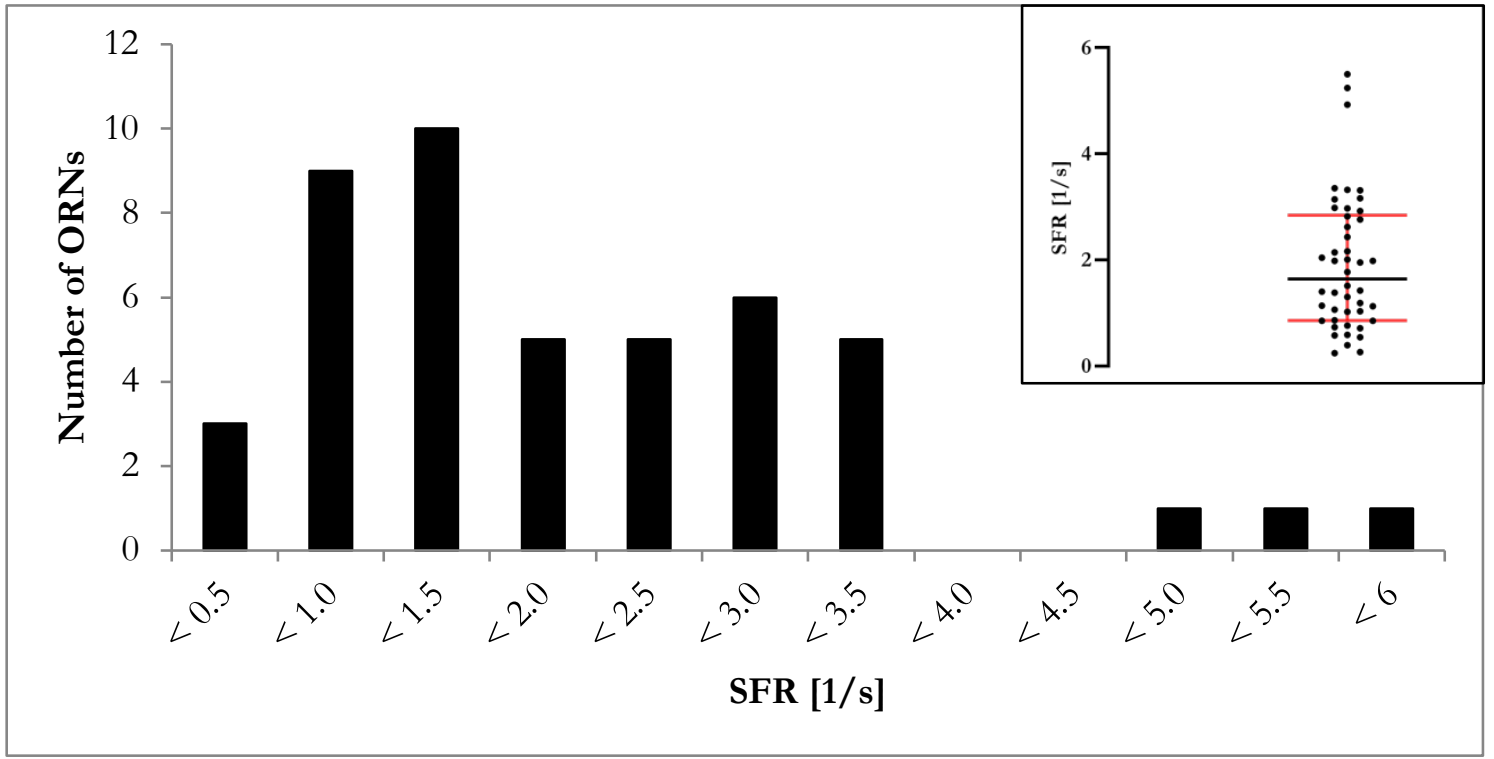

Figure 5 Distribution of averaged spontaneous firing rates

A $\mid$ The spontaneous firing rates varied considerably among the 46 ORNs recorded. The y-axis represents the absolute number of ORNs being counted for each SFR class (bin width $=0.5 \mathrm{~s}$ ). Some cells were firing less than 1 AP per second (see Table 3 below), others more than 5 per second. On average 1.64 APs per second were generated but most cells $(n=10)$ had a firing rate between 1.00 and 1.49 APs per second. The inserted box plot on the right upper side illustrates the averaged spontaneous firing activity of every single ORN. Median (1.64/s) with first and third quartile (in red) are displayed. SFR = spontaneous firing rate, $\mathrm{AP}=$ action potential.

Table 3 SFRs of 46 ORNs

\begin{tabular}{|cccccc|} 
& Minimum & 1st Quratile & Median & 3rd Quartile & Maximum \\
\hline Spikes/s & 0.24 & 0.90 & 1.64 & 2.81 & 5.5 \\
\hline
\end{tabular}

As a characteristic feature of the Poisson distribution the mean event rate is identical to the variance, i.e. $\lambda=\sigma^{2}$. In ORNs with a significantly different distribution of the SFR than expected in a simple Poisson process, the mean-to-variance ratio of the SFR was either much higher or much lower than 1. 


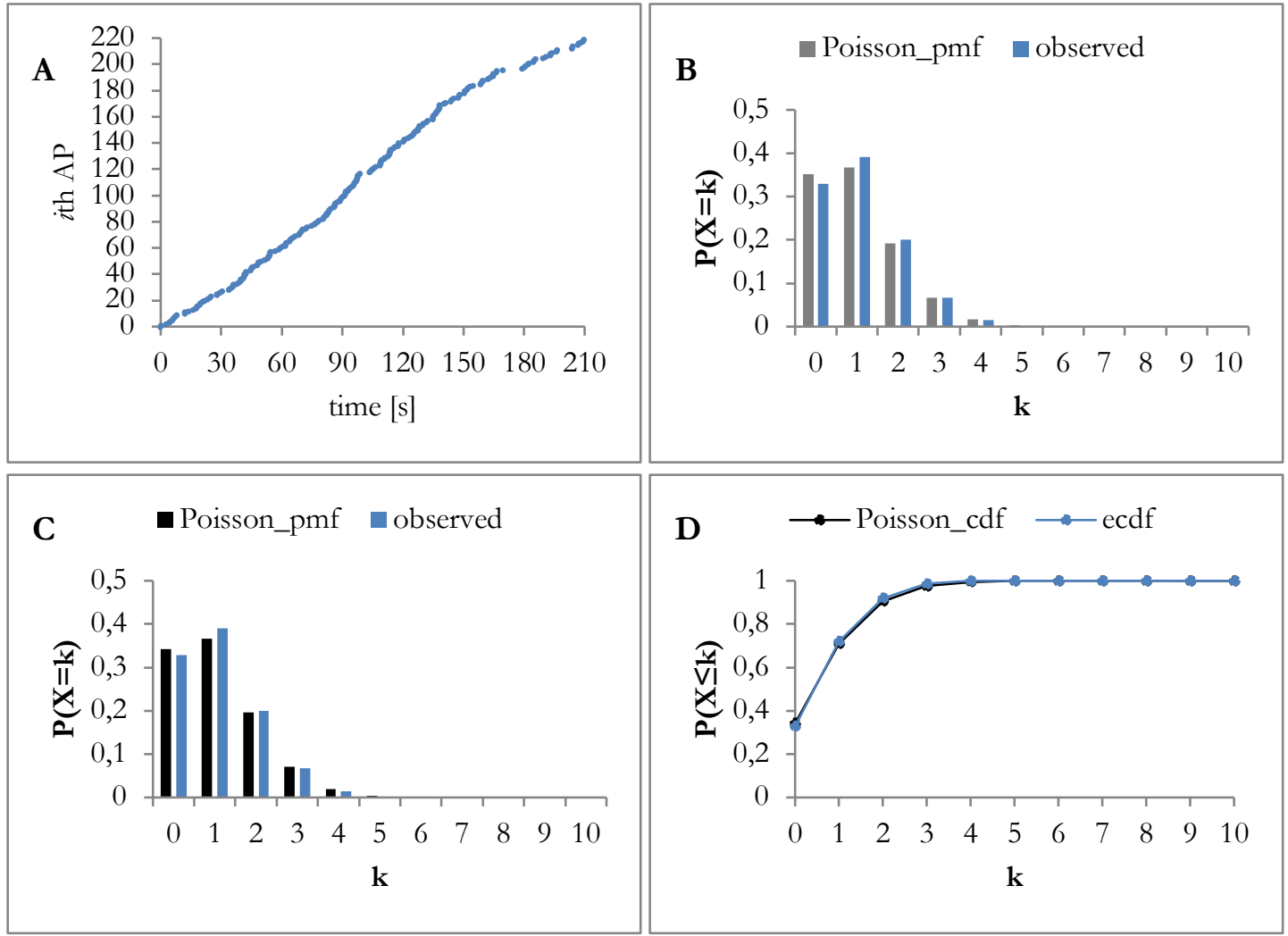

Figure 6 SFR and Poisson distribution

A| Data points for 1 ORN were recorded in the steady state. Sequences of $30 \mathrm{~s}$ before each of the 7 stimulus applications were linked up and yielded a nearly linear increase of the number of APs over the recording time. For this particular ORN 220 APs were recorded in 210.22 s, i.e. the mean SFR was $1.05 \mathrm{~s}^{-1} . \mathbf{B} \mid$ This histogram represents the observed relative frequencies (y-axis) of number of APs per $1 \mathrm{~s}$-bin width (k) compared to the estimated Poisson probability mass function with $\lambda=\operatorname{SFR}=1.05 \mathrm{~s}^{-1}$. The observed counts of APs per bin matched the theoretical model well. C $\mid$ Adjusting the Poisson pmf such that $D=\sup \left|F_{0}(k)-S_{n}(k)\right|$ is minimal led to a slightly different event rate $\lambda=1.07 \mathrm{~s}^{-1} . \mathbf{D} \mid$ The cumulative distribution functions computed from the theoretical and empirical data points were nearly identical (no statistical difference in the KST, $\alpha=$ $1 \%)$. Pmf $=$ probability mass function, $(\mathrm{e}) \mathrm{cdf}=($ empirical $)$ cumulative distribution function.

In order to get an overview on all tested ORNs the mean-to-variance ratio, was plotted for each ORN (Figure 7). As expected, for the majority of ORNs the mean SFR was nearly equal to the variance $\left(\mu \approx \sigma^{2}\right)$. However, in a few neurons the ratio was relatively either low (i.e. high variance in relation to the mean SFR) or high (i.e. little variance). 


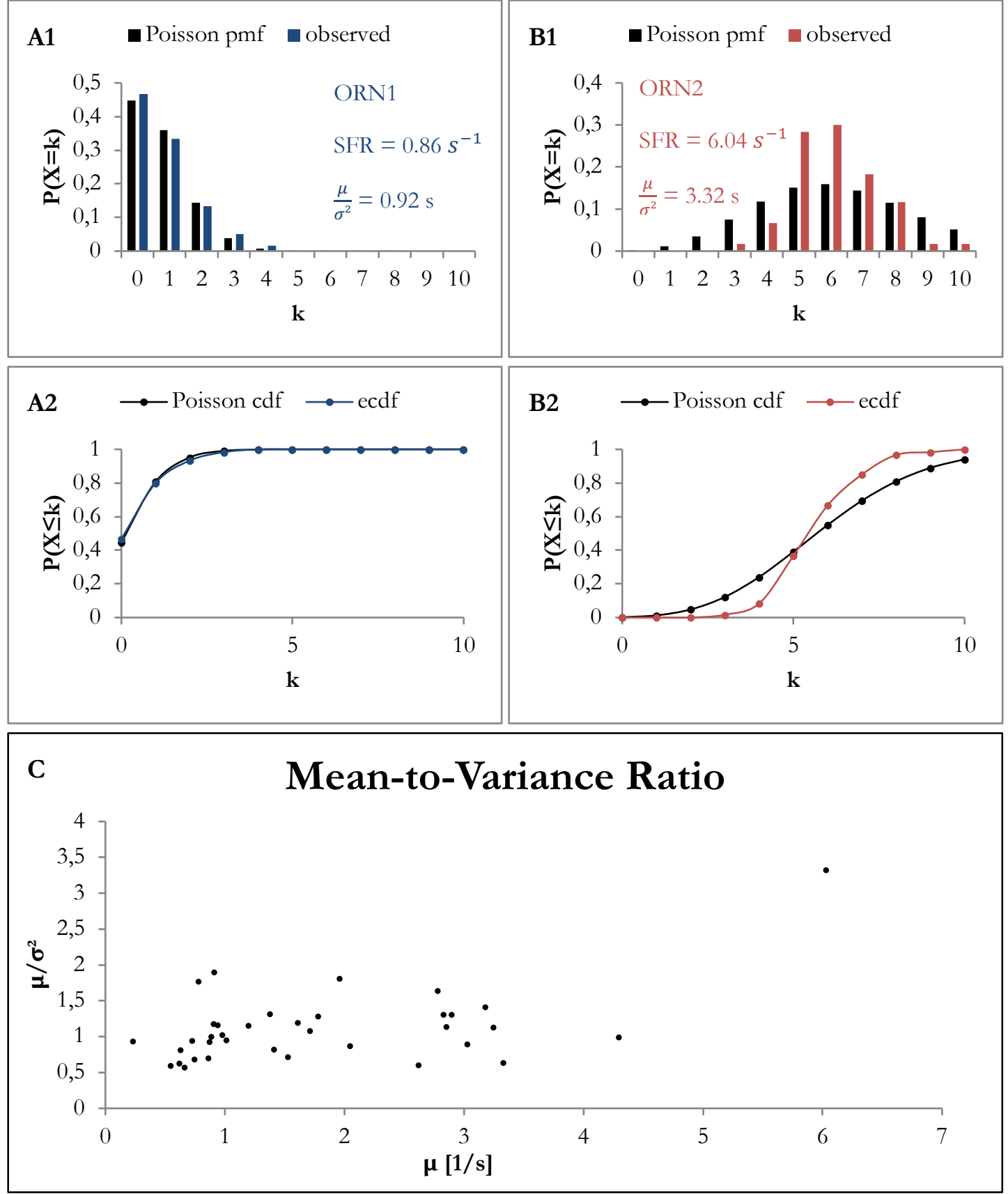

Figure 7 Mean-to-variance ratio

A| A1 and A2 represent an exemplary arginine sensitive ORN with a SFR of $0.86 \mathrm{~s}^{-1}$ and the corresponding theoretical Poisson pmfs and cdfs. The mean-to-variance ratio in this neuronal activity is $0.92 \mathrm{~s}$; the spontaneous activity is a Poisson process. B $\mid \mathrm{B} 1$ and B2 correspond to A1 and A2 for a different arginine-sensitive ORN with SFR $=6.02 \mathrm{~s}^{-1}$ and $\frac{\mu}{\sigma^{2}}=3.32 \mathrm{~s}$. B2 shows that the empirical cumulative distribution function differed significantly from a Poisson process (KST, significant at $\alpha=0.05)$. C $\mid$ Mean-to-variance ratios for 36 analyzed ORNs. 60 s of spontaneous activity were taken to calculate the mean and variance in spontaneous activity. 
ORNs with a low mean-to-variance ratio exhibit a high variance in the basal activity. In Figure 8 an example of an arginine-sensitive ORN with a high variance (SFR $=2.63 \mathrm{~s}^{-1}$, variance $\mathrm{V}=4.38 \mathrm{~s}^{-2}$ and a mean-to-variance ratio $0.6 \mathrm{~s}$ ) is demonstrated. Plotting the probability mass function of the corresponding Poisson distribution shows that the observed data points obviously represent 2 different distributions with 2 maxima, one at $\mathrm{k}$ $=1$ and another at $\mathrm{k}=4$ ( 2 arrows in subfigure $\mathrm{B})$, indicating 2 peak interspike interval lengths at $\tau_{1} \approx 1000 \mathrm{~ms}$ and $\tau_{2} \approx 250 \mathrm{~ms}$ (bin width $1 \mathrm{~s}$ ). Moreover, the raw spike train (subfigure A) already indicates at least 2 different phases in spike generation: one phase with low SFRs and a second one with higher SFRs. Similar results were found for 4 ORNs with the lowest $\frac{\mu}{\sigma^{2}}$ ratios (subfigure C).

A
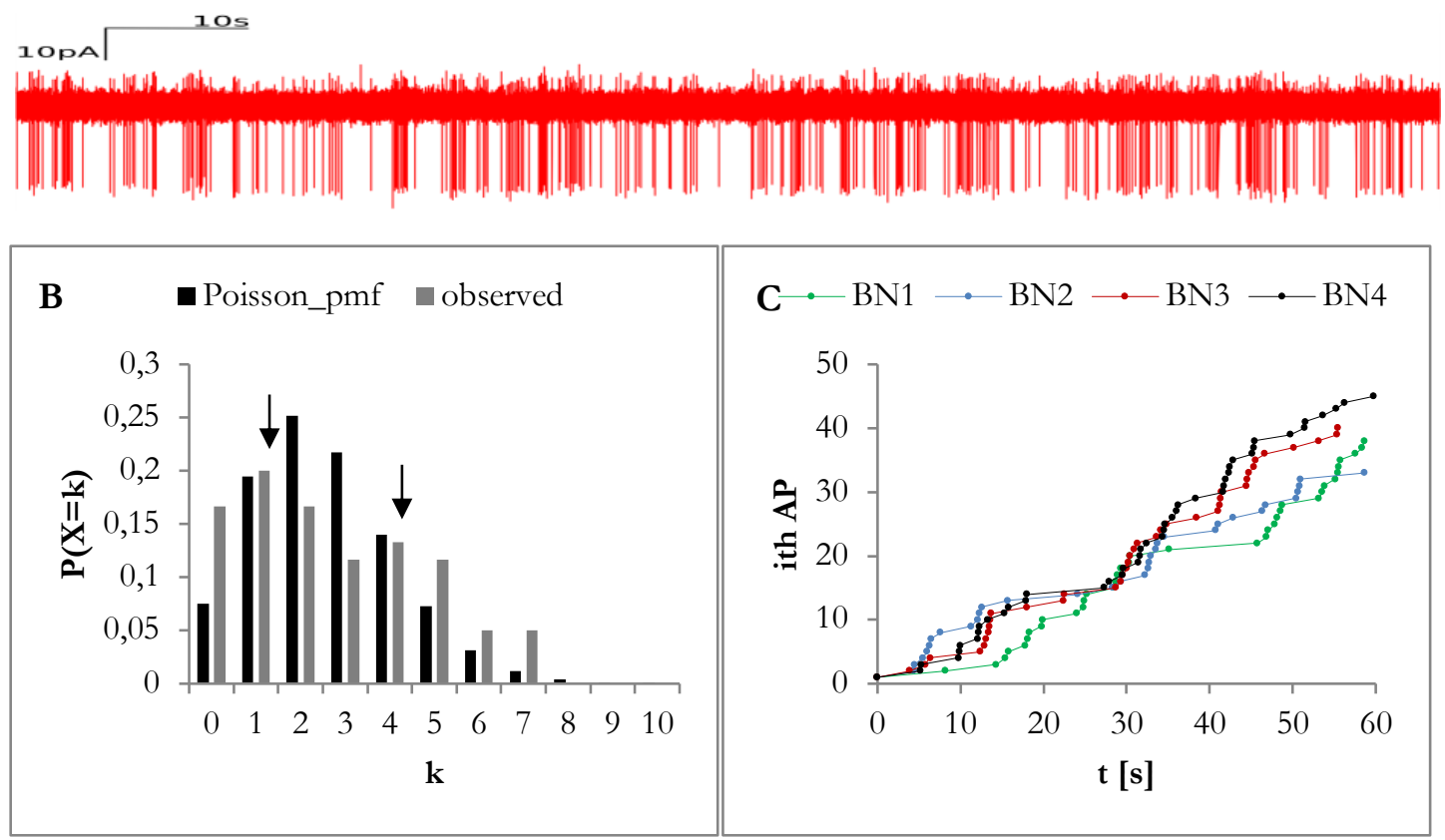

Figure 8 ORNs with high variance of spontaneous activity

A| Raw recording train of an arginine-sensitive ORN with a SFR of $2.63 \mathrm{~s}^{-1}$ and a $\frac{\mu}{\sigma^{2}}$ ratio of $0.6 \mathrm{~s}$. The spike train consists of phases with low SFR and intermittently high AP rates. B| Upon the mean SFR the event rate $\lambda$ was estimated. The corresponding Poisson probability mass function significantly differed from the actual data (KST, $\alpha=5 \%$ ). The distribution of the observed data apparently consisted of 2 overlapping distributions with 2 peaks at $\mathrm{k}=1$ and $\mathrm{k}=4$ (arrows), respectively. C| Excerpt of 60s spontaneous activity recordings in 4 different ORNs showing sequences of mostly 2-4 highly frequent APs sequences overlapping a "baseline" SFR. All 4 ORNs showed "bursting" behavior and had therefore a high variance in the SFR which led to a low $\frac{\boldsymbol{\mu}}{\boldsymbol{\sigma}^{2}}$ ratio. $\mathrm{BN}=$ bursting neuron. 
A slow baseline activity was thereby overlapped by sequences of mostly 2 - 4 APs of high frequency. The next question aimed at discovering whether the slow baseline activity became a simple Poisson process if the high-frequency "bursts" were eliminated from the SFR. Furthermore, it was interesting to know if the observed distributions, i.e. the high and low frequent firing rates, were simple Poisson processes themselves. However, in order to analyze both distributions separately it would have needed to arbitrary define "high SFR" phases and "low SFR" phases, respectively. To circumvent this problem, a new approach to this question was necessary. As the SFRs of most ORNs were Poisson processes, it was legitimate to assume that the number of interspike intervals $\tau$ per bin (e.g. $1 \mathrm{~ms}$ or $10 \mathrm{~ms}$ ) was a random Poisson process as well (Figure 9).

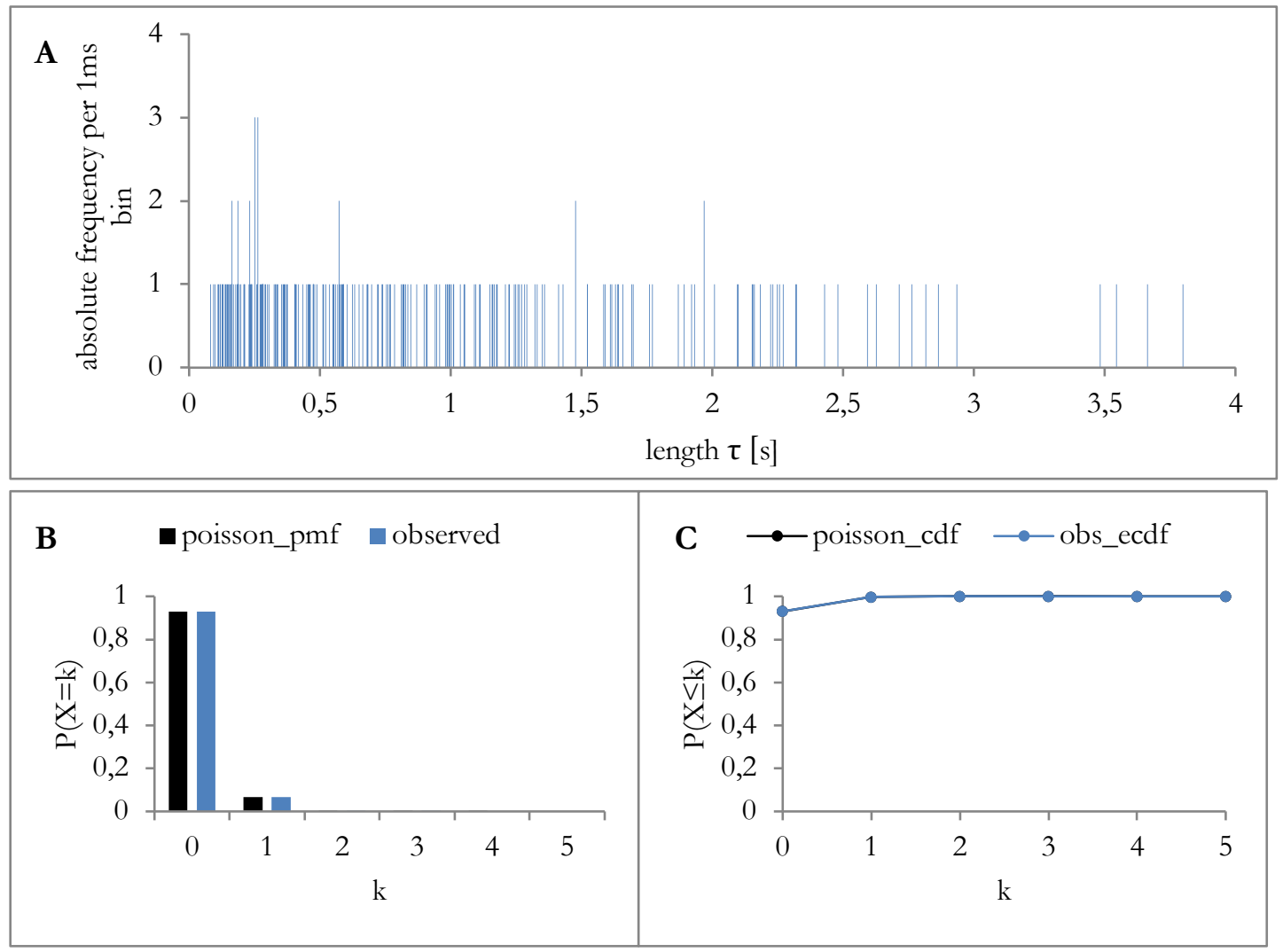

Figure 9 Distribution of interspike interval length $\tau$

A | All interspike intervals $\tau$ from 1 ORN (identical with ORN in Figure 6 ) were first sorted from shortest to longest interval and then the absolute frequency in each $1 \mathrm{~ms}$-bin was determined. The

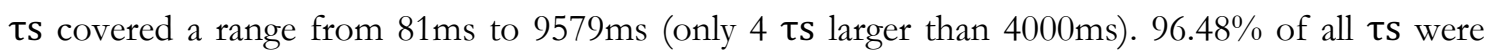
shorter than $3000 \mathrm{~ms}$, some $\tau$ s were identical and 2 ts occurred even 3 times in a bin. $\mathbf{B} \mid$ The averaged number of $\tau$ s per bin $(\approx 0.073)$ was used to estimate the event rate $\lambda$. The Poisson model and the observed data were nearly identical. $\mathbf{C} \mid$ The cumulative distribution functions of Poisson model and empirical data did not show statistical significant differences. $\mathrm{pmf}=$ probability mass function, $\mathrm{cdf}=$ cumulative distribution function, $\mathrm{k}=$ number of $\tau$ per $1 \mathrm{~ms}$ bin. 
The advantage of this approach was to extract temporal information of the raw data and to simply focus on the length of $\tau$, independently of the time the corresponding APs were generated. So, if the raw data consisted of 2 overlapping processes, 2 different distributions in the length of the interspike intervals $\tau$ should be expected.

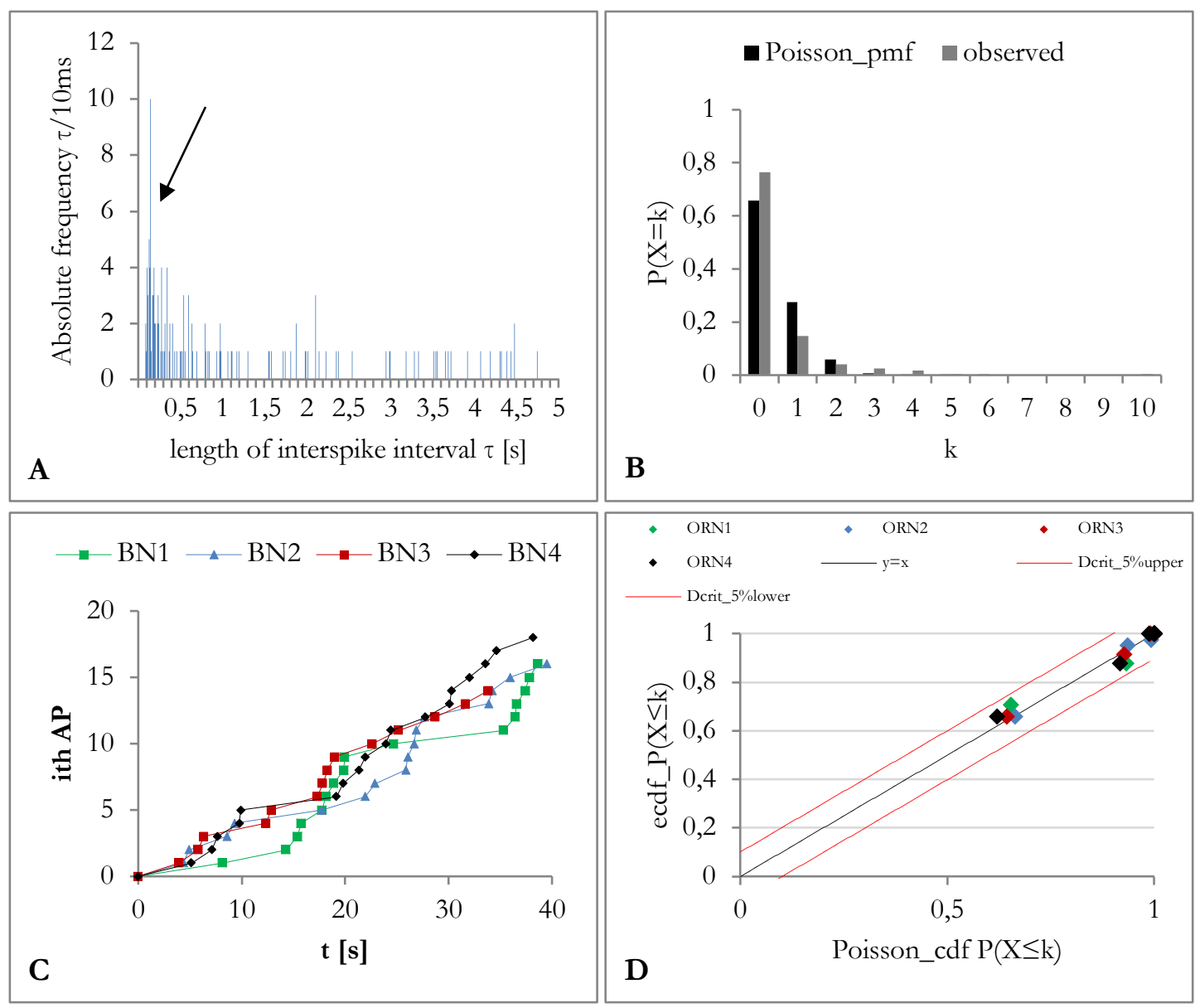

Figure 10 Bursting neurons

A| The cumulative distribution of interspike intervals $\tau$ s of 4 bursting neurons. All $\tau$ s were sorted from shortest to longest interval and categorized in 10 ms-bins ( $\mathrm{x}$-axis). The absolute frequency (yaxis) showed a local maximum in the short interspike interval range of $\tau<350 \mathrm{~ms}$ (arrow). Thereby, 10 interspike intervals had a length of $130 \mathrm{~ms}$ to $140 \mathrm{~ms}$. B | Theoretical and empirical probabilities for the absolute frequency of $\tau$ per bin. The observed data (all interspike interval lengths up to $3000 \mathrm{~ms}$, covering $80.92 \%$ of all intervals) were statistically different than expected in a Poisson process (KST, $\alpha=5 \%$ ). C $\mid$ Absolute frequency of AP (y-axis) and recording time (x-axis) after eliminating the highly frequent bursts from spontaneous activity (compare subfigure $\mathrm{C}$ in Figure 8). $\mathbf{D} \mid$ Empirical cumulative function (y-axis) plotted against the theoretical Poisson cumulative distribution function. After eliminating the high frequent AP sequences with $\tau_{\text {crit }}<$ $350 \mathrm{~ms}$ the SFR of the corresponding ORN became a Poisson process. 
In Figure 9, the Poisson character in the distribution of the length of interspike interval $\tau$ has been demonstrated. Thereby, "event rate" is corresponding to the number of interspike intervals that have been categorized in a $1 \mathrm{~ms}$-bin. Plotting the interspike intervals of the 4 "bursting" ORNs over $60 \mathrm{~s}$ (ORNs from Figure 8) revealed a local maximum at approximately $\tau<350 \mathrm{~ms}$ and a global maximum at $130 \mathrm{~ms}<\tau \leq 140 \mathrm{~ms}$ (Figure 10). Assuming all bins containing at least 4 interspike intervals were contributing to the highly frequent bursts in all 4 ORNs (because for longer interspike intervals there were no bins containing 4 or more interspike intervals) then it is possible to set an (artificial) cut-off value at $\tau \approx 350 \mathrm{~ms}$. This way, the "bursts" in all 4 ORNs (60 s recording time) consisted of 21 couplets, 10 triplets and 4 longer sequences with a maximum of 6 APs per sequence (total number of APs $n=152$ ). By eliminating all AP sequences with $\tau_{\text {crit }}=A_{n+1}-A P_{n}<$ $350 \mathrm{~ms}$ from the raw spiking train, indeed, the SFR in the initially tested neuron became a simple Poisson process (subfigure $\mathrm{C}$ and $\mathrm{D}$ ) again.

When the length of $\tau$ for short and long interspike intervals were tested if they had a Poisson character, they revealed that in $2 / 4$ bursting neurons the short interspike intervals, i.e. $\tau<350 \mathrm{~ms}$, didn't have Poisson character whereas in all neurons longer interspike intervals did have a Poisson character (data not shown).

In case of the ORN with a high $\frac{\boldsymbol{\mu}}{\boldsymbol{\sigma}^{2}}$ ratio (Subfigure B1 and B2 in Figure 7) the same way of analysis, i.e. analyzing the length of interspike interval $\tau$, showed likewise a local maximum of short interspike intervals for $\tau<200 \mathrm{~ms}$ (Figure 11). Approximately $71 \%$ of all intervals were shorter than $175 \mathrm{~ms}$, the absolute maximum with 7 ts per bin lay in the range of 110 - $130 \mathrm{~ms}$. The distribution of the lengths of interspike intervals was significantly different than expected in a Poisson process for all $\tau<175$ ms (i.e. the last bin with 4 ts per 1 msbin), whereas longer interspike intervals did have Poisson character (subfigure B). However, for this neuron it was not possible to extract the high frequent AP sequences from the raw spiking train as they made up approximately $71 \%$ of all data points.

In summary, the SFRs of all analyzed ORNs were modeled by a Poisson distribution. In the majority of ORNs $(35 / 36)$ SFR means and variances were in the same range, i.e. $\frac{\boldsymbol{\mu}}{\boldsymbol{\sigma}^{2}} \approx 1$, and therefore could be modeled by a simple Poisson process. In one case, the SFR became a simple Poisson Process by eliminating highly frequent sequences of APs with critical interspike interval length $\tau_{\text {crit }}<350 \mathrm{~ms}$ in case of a low $\frac{\mu}{\sigma^{2}}$ ratio. For the receptor neuron with a high ratio it was possible to show that short interspike interval lengths do not have Poisson character but contribute to approximately $71 \%$ of the data points. 

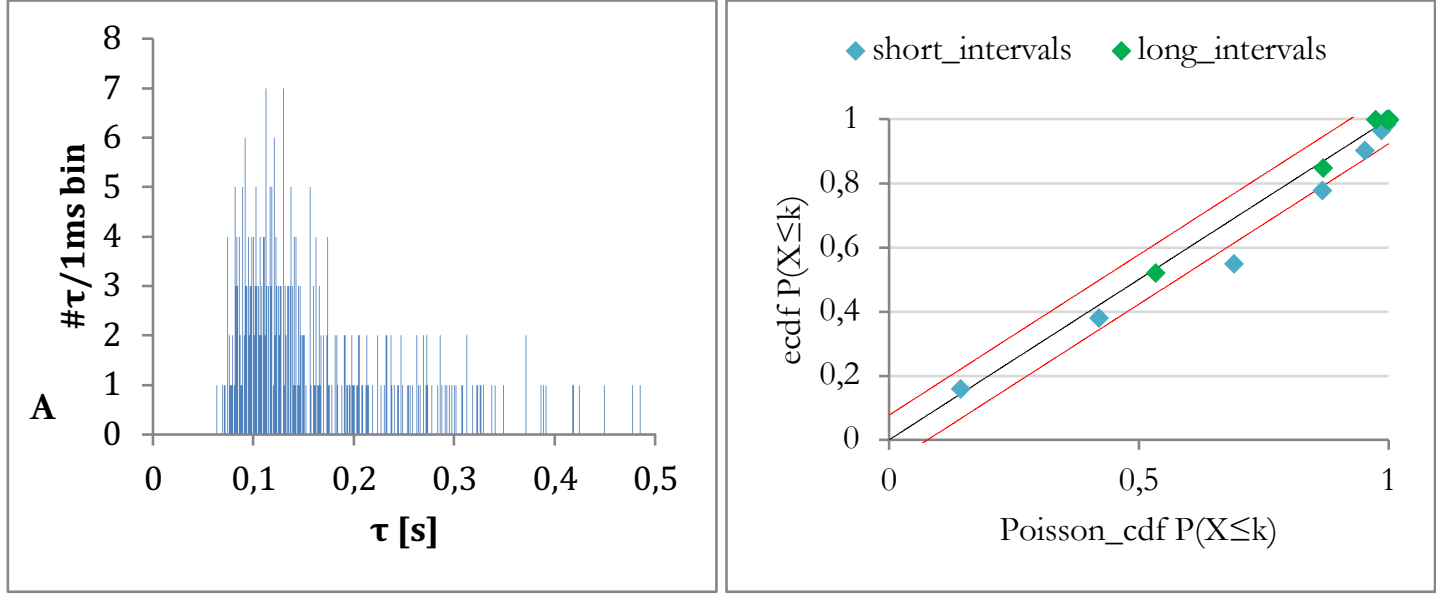

Figure 11 A fast firing ORN

A Distribution of length of interspike interval $\tau$ in an ORN with high $\frac{\boldsymbol{\mu}}{\boldsymbol{\sigma}^{2}}$ ratio (identical to the ORN in B.1 and B.2 Figure 7). There is a local maximum for short interspike intervals with $\tau<$ $175 \mathrm{~ms} . \mathbf{B} \mid$ Comparison of short (63 ms $<\tau<175 \mathrm{~ms})$ and longer $(175 \mathrm{~ms}<\tau<287 \mathrm{~ms})$ interspike interval ranges. The observed data in the short interval range (blue points) were significantly different than expected for a Poisson process (red linear curves represent upper and lower limits for critical difference $\mathrm{D}_{\text {crit }}$ at $1 \%$-level in the Kolmogorov-Smirnov-test). Longer interspike intervals (same interval range width of $112 \mathrm{~ms}$ ) had a Poisson character (green points).

As a last step, the converging inputs of 4 different SFR sources were simulated for a virtual glomerulus (Figure 12). The input of similarly tuned ORNs, i.e. similar in SFR range (and sensitive to the same amino acid) resulted in a simple Poisson process at the "OB level".

arginine

- Poisson_pmf observed

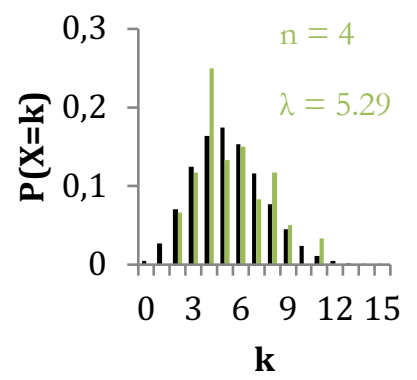

methionine

- Poisson_pmf abserved

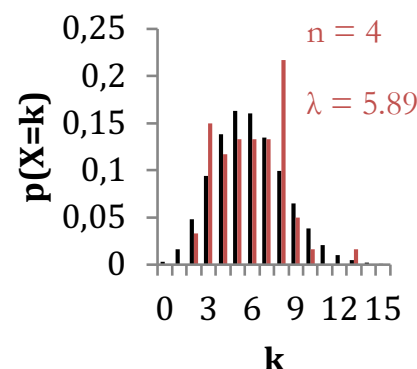

alanine

— Poisson_pmf o observed

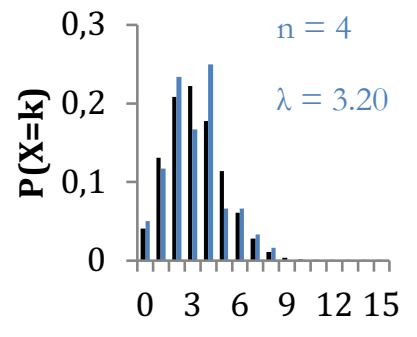

$\mathbf{k}$

Figure 12 Simulation of glomerular input

12 ORNs were chosen to simulate the input into 3 virtual glomeruli. Thereby for each amino acid (arginine, methionine and alanine) 4 representative ORNs were chosen such that their SFRs were adding up in 1 s-bins. Although only 4 ORNs were chosen to simulate the input to a glomerulus, the resulting distribution was conform to a theoretical Poisson process. 


\subsubsection{ORNs have different thresholds}

As a next step the single amino acid solution was applied in increasing concentrations in order to record the spiking patterns in reaction to different concentrations of stimuli. Thereby, the lowest concentration, $10 \mathrm{nM}$, was applied first and followed by the next highest concentration and so forth (Figure 13).

A
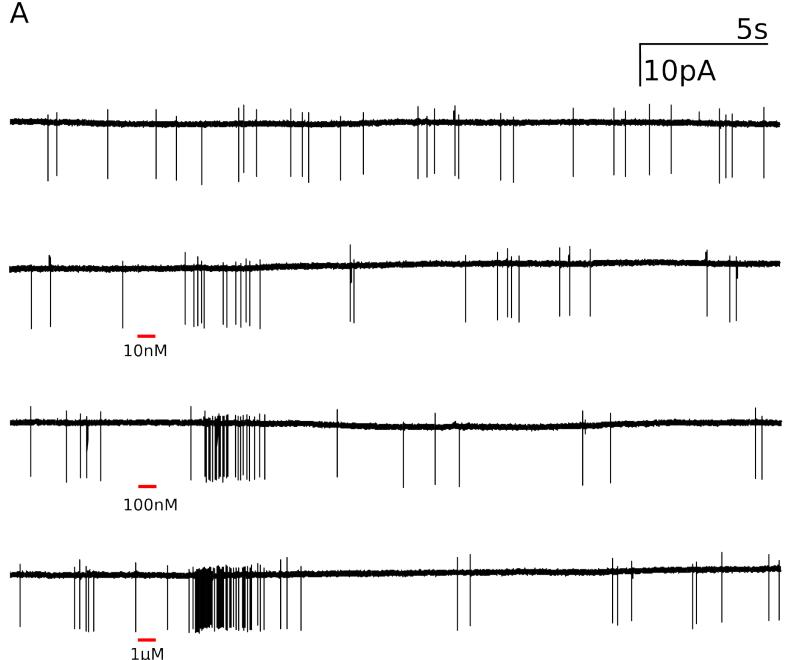

$\overline{1 \mu M}$
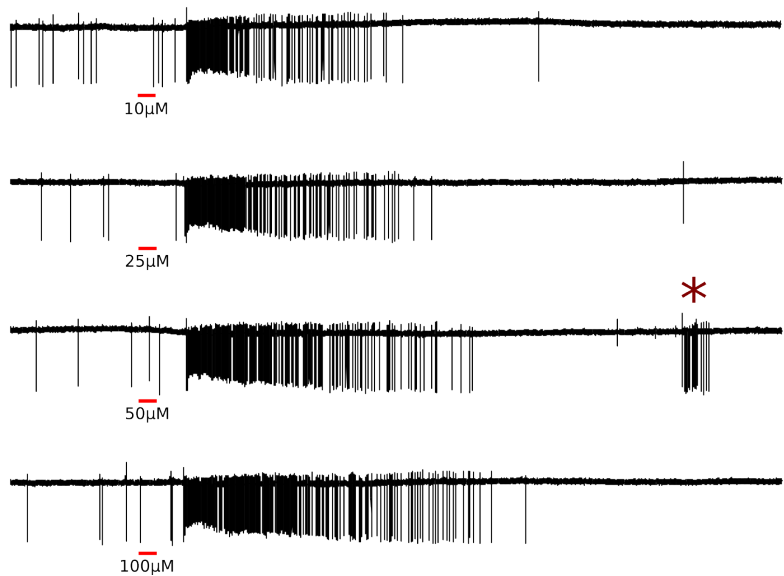

B

$\frac{5 \mathrm{~S}}{10 / \mathrm{s}}$

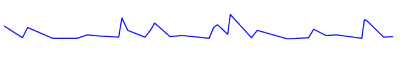

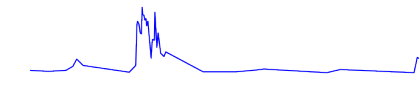

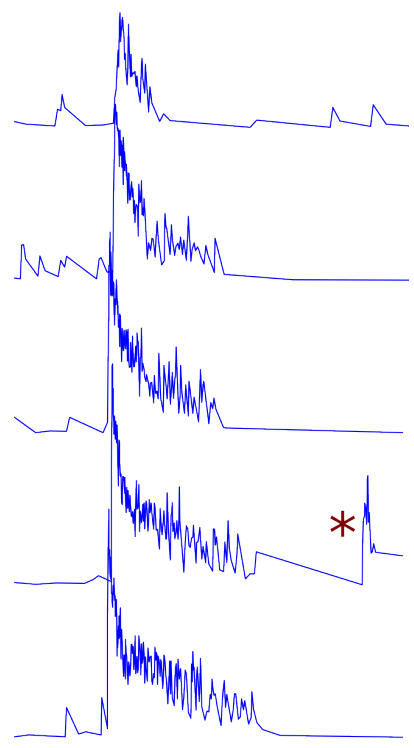

Figure 13 Stimulus-induced responses in an arginine-sensitive ORN

A| This figure shows the spiking train of an exemplary ORN in the cell-attached mode. Thereby, the first row shows the spontaneous activity of the cell, while the other rows illustrate the increasing spiking rate after stimulus application. Notably, the increasing firing rates were followed by a firing "pause" of the ORN. This pause increases with increasing stimulus application but can be interrupted by ringer control application which presumably washes out remnant amino acid solution in the application tube at high concentrations (see *). Such a phenomenon is seen also seen for $100 \mu \mathrm{M}$ concentrated solution but cut out in this figure due to space limitations. B $\mid$ The curves represent the resulting firing rates as the instantaneous firing frequency, i.e. 1 divided by the interspike interval $\tau(1 / \tau)$. The maximum firing rate $f_{\max }$ is thereby $7.79 s^{-1}$ for $10 \mathrm{nM}$ and $57.14 \mathrm{~s}^{-1}$ for $100 \mu \mathrm{M}$ corresponding to a minimum $\tau$ of $128.4 \mathrm{~ms}$ and $17.5 \mathrm{~ms}$, respectively. 
The applied concentrations covered a 5-log wide range. The ORNs showed a striking difference in the detection thresholds for the applied stimulus; some ORNs already responding to the lowest applied concentration, whereas other ORNs responded only to the highest applied concentration. $6.5 \%$ of the ORNs responded to concentrations in the nanomolar range (10 $\mathrm{nM}$ and $100 \mathrm{nM}$ ) whereas all ORNs responded to concentrations as high as $50 \mu \mathrm{M}$ or higher (Figure 14).
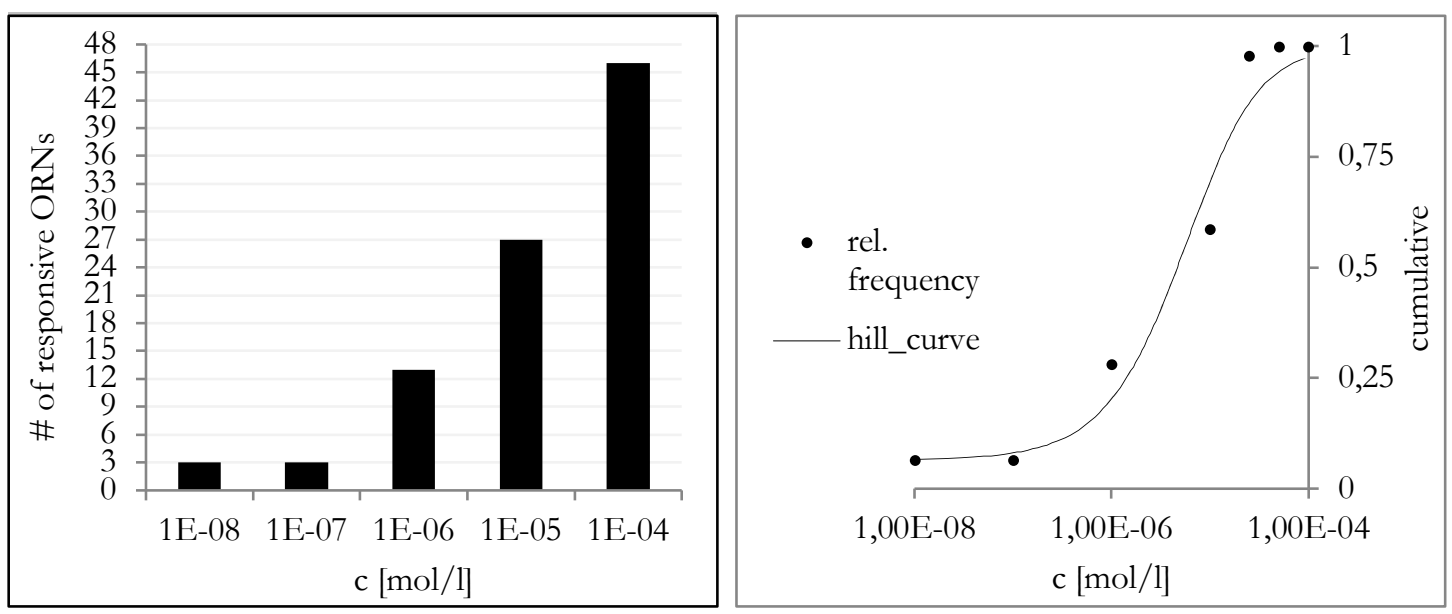

Figure 14 ORNs' response thresholds

A Absolute numbers of ORNs responsive to different concentrations. 3 out of 46 ORNs responded to the lowest applied concentration, $10 \mathrm{nM}$, whereas all ORN responded to amino acid solutions at $100 \mu \mathrm{M}$ concentration. $57.4 \%$ (27 out of 46 ) of the cells responded to $10 \mu \mathrm{M}$ and higher concentrated solutions whereas 13 ORNs were responsive to $1 \mu \mathrm{M}(27.7 \%)$ and higher suggesting that $50 \%$ of the cells respond to a fictive concentration ranging between $1 \mu \mathrm{M}$ and 10 $\mu \mathrm{M}$ ( $=$ threshold concentration in $50 \%$ of the ORNs, EC50-value). The applied concentrations spanned a 5-log scale wide range and revealed that ORNs' sensitivity can vary extensively. B Relative portions of responsive ORNs to the applied stimuli. In a part of the ORNs $(n=15)$ the stimuli were also applied at concentrations of $25 \mu \mathrm{M}$ and $50 \mu \mathrm{M}$. All ORNs responded to $50 \mu \mathrm{M}$, just 1 ORN was not responsive to $25 \mu \mathrm{M}$ concentrated amino acid solution. These 2 additional data points helped to define the shape of the dose response curve as sigmoidal. The data points (black data points) are fitted by the sigmoidal function $y(c)=\max -\frac{\max -\min }{1+\left(\frac{c}{E C 50}\right)^{m}}, c=$ concentration, EC50 $=$ $8.8 \mu \mathrm{M}$ and $\mathrm{m}=1.49$. The fictive EC50 value is thereby calculated at $8.8 \mu \mathrm{M}$.

The majority of cells $(71.7 \%)$ was activated by stimuli concentrations of $10 \mu \mathrm{M}$ and above while $28.3 \%$ of the ORNs responded to $1 \mu \mathrm{M}$ and higher suggesting that more than $50 \%$ of the cells responded to a fictive concentration ranging between $1 \mu \mathrm{M}$ and $10 \mu \mathrm{M}$ (EC50value). This value can be calculated by fitting the data points with a sigmoidal function, resulting in a calculated value at $8.8 \mu \mathrm{M}(\mathrm{m}=1.49)$. 
The differences in the sensitivity of the cells is not due to the amino acids themselves as ORNs responsive to the same amino acid showed different thresholds of responses; e.g. some ORNs responded to arginine solutions at $10 \mathrm{nM}$ whereas others - that were responsive to arginine - did only respond to concentrations higher than $10 \mu \mathrm{M}$. When taking the EC-50 value as a distinguisher of (threshold) sensitivity then the receptor neurons can be categorized as low-sensitive (i.e. stimulus concentration needed to evoke a response is higher than the EC-50 value) or high-sensitive (stimulus concentration below EC-50 value will elicit a response). Accordingly, 36/46 ORNs were low-sensitive ORNs whereas 13 ORNs were high-sensitive ORNs (Figure 15).

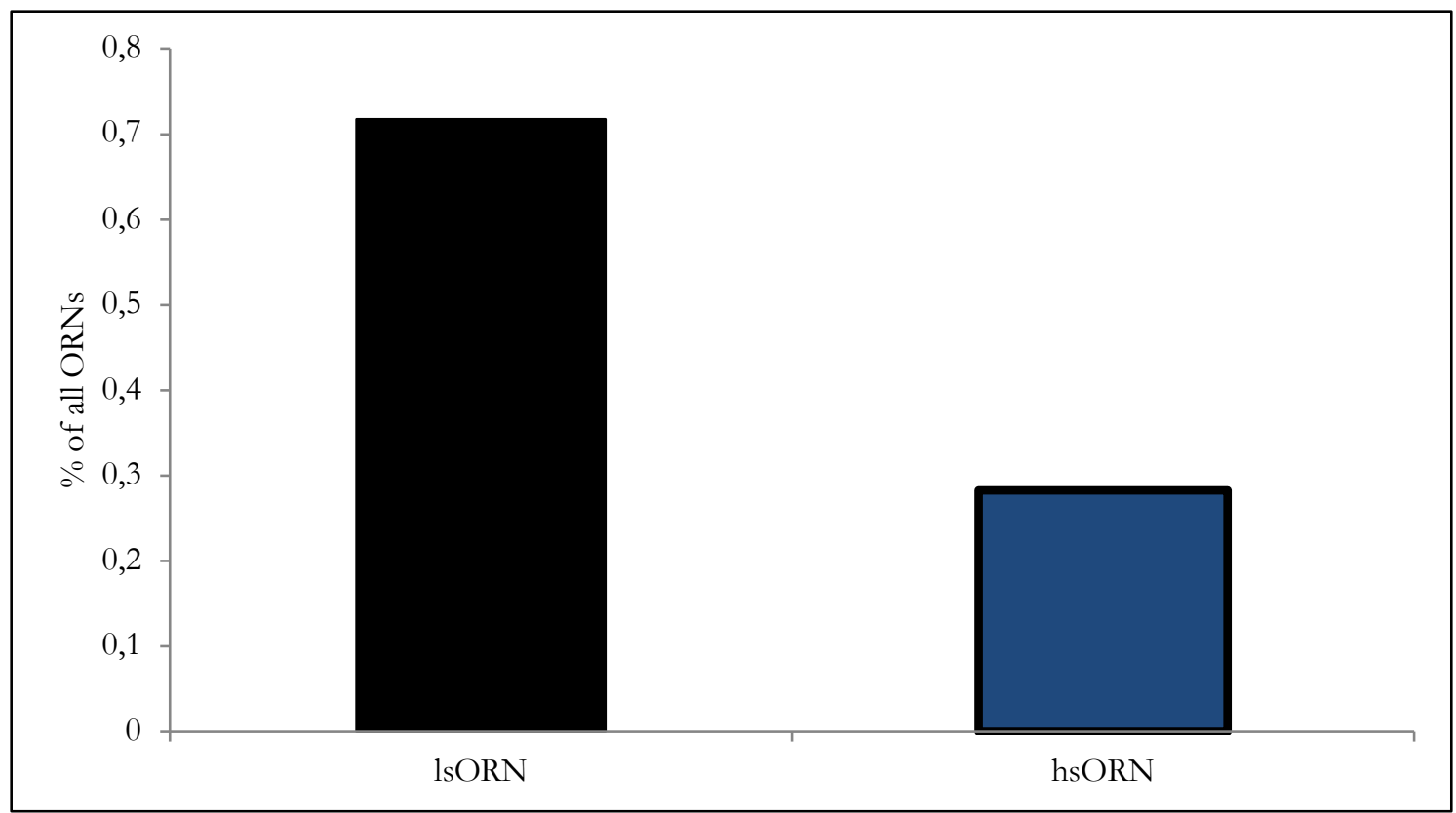

Figure 15 Categories of sensitivity

33 ORNs were low-sensitive $(71.7 \%)$ compared to 13 ORNs $(28.3 \%)$ responding to concentrations as low as $1 \mu \mathrm{M}$. ls $\mathrm{ORN}=$ low-sensitive odorant receptor neuron with $\mathrm{c}($ threshold) $\geq 10 \mu \mathrm{M}$, hsORN $=$ high-sensitive ORN with $\mathrm{c}($ threshold $) \leq 1 \mu \mathrm{M}$.

\subsubsection{Inverse proportionality of SFRs and sensitivity}

The first two observations indicated that ORNs had different spontaneous firing rates and were differently sensitive to the same stimulus. It was therefore interesting to investigate whether both features were correlated with each other. To answer this question the ORNs were categorized according their spontaneous firing rates into 3 nearly equally sized groups: slow firing ORNs (sfORN, $\mathrm{n}=16$ ), fast firing ORNs (ffORN, $\mathrm{n}=16$ ), and an intermediate group (imORN, $n=14$ ). Then, the portion of lsORNs and hsORNs were determined in the three groups of ORNs: sfORNs, ffORNs and imORNs (Figure 16). Thereby, slow firings ORNs consisted of almost counterbalanced portions of low-sensitive 
and high-sensitive cells. In contrast, only 1 out of 16 ORNs was high-sensitive among all ffORNs. The intermediate group was represented by a similar distribution of lsORNs and hsORNs as already seen for the total number of all ORNs (Figure 15). The differences in the groups were statistically significant $\left(\chi^{2}\right.$-test, $\left.\mathrm{p}=0.0439\right)$.

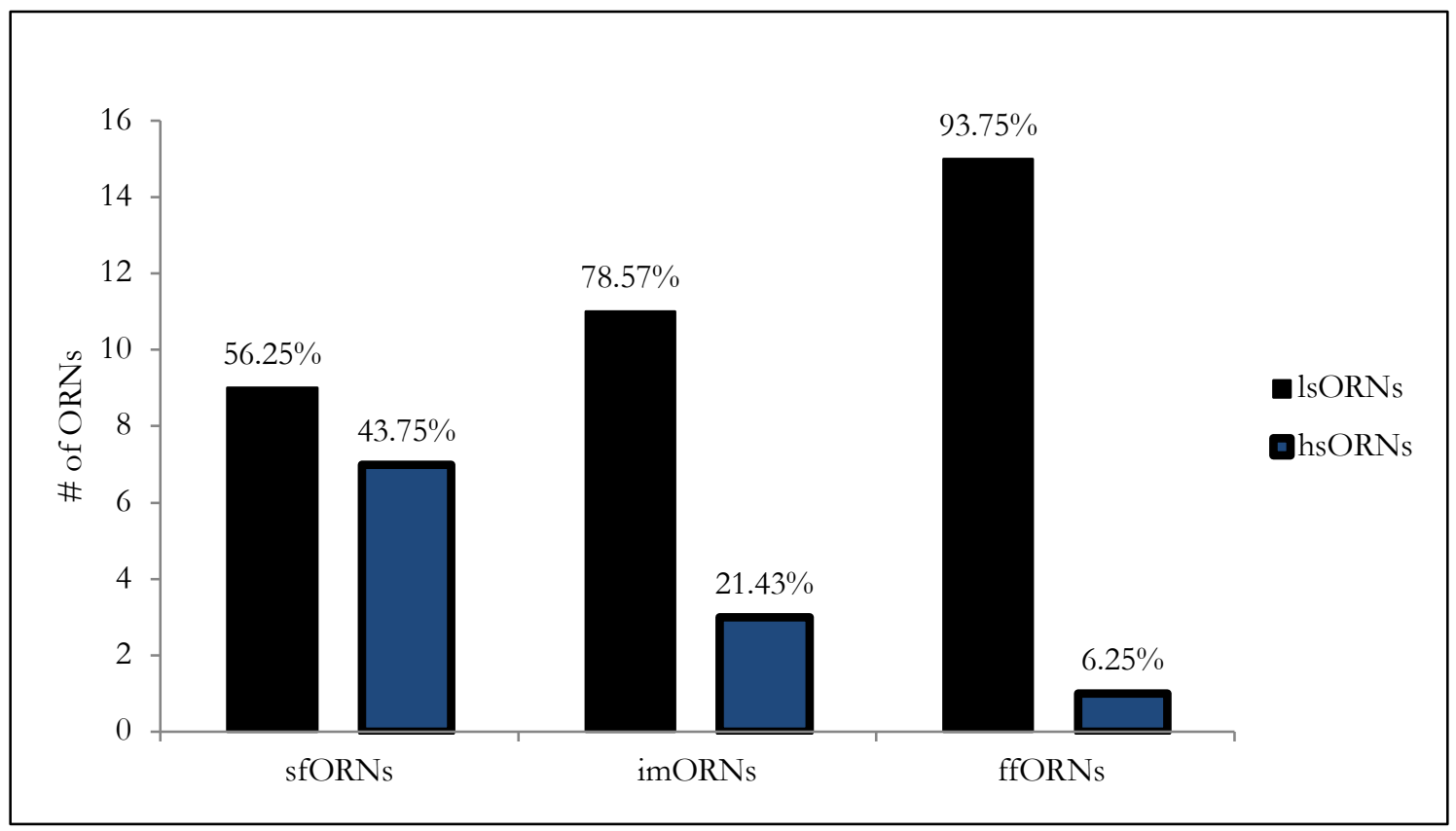

Figure 16 Inverse proportionality of SFRs and sensitivity

Differences in signal detection thresholds in ORNs with different SFRs. Only 1 ffORN was highly sensitive whereas 15 were low-sensitive ( $n=16$; $93.75 \%$ vs. $6.25 \%$ ). In contrast, the sfORNs ( $n=$ 16) consist of an almost counterbalanced portion of low-sensitive and high-sensitive neurons. The imORNs $(n=14)$, however, show a similar distribution of lsORNs and hsORNs as already seen for all ORNs (see Figure 15). Therefore, sfORNs are more sensitive than ffORNs (statistically significant in the $\chi^{2}$-test, $\left.\mathrm{p}=0.0439\right)$. sfORN $=$ slow firing odorant receptor neuron, imORN $=$ intermediate odorant receptor neuron, ffORN $=$ fast firing odorant receptor neuron, $1 \mathrm{sORN}=$ low-sensitive odorant receptor neuron, hsORN = highly sensitive odorant receptor neuron.

Arguably, the applied amino acids may have directly affected ORN sensitivity. ORNs may be less sensitive to some sort of amino acids and therefore the observed relation could be due to the stimulus itself and not due to the SFR. Another argument that could possibly explain the observation is the fact that responses to low concentrations can be more easily detected in slow firing ORNs compared to ORNs with a high basal activity and therefore an unfavorable signal-to-noise ratio.

To exclude both possibilities, first, the portions of lsORNs and hsORNs were analyzed for ORNs that were sensitive to the most frequently applied amino acids. Furthermore, in 
order to understand the distribution, it was also important to show the portion of sfORNs, imORNs and ffORNs for each single amino acid application (see Figure 32 in Appendix). Arginine and methionine sensitive ORNs revealed a higher proportion of lsORNs (54.55 $\%$ and $70 \%$, respectively). Thereby, the methionine-sensitive ORNs were mostly reminiscent of the overall distribution of lsORNs and hsORNs in all tested ORNs (lsORNs to hsORNs: $70 \%$ to $30 \%$ in methionine sensitive ORNs versus $72 \%$ to $28 \%$ in all ORNs). Those finding were in line with the almost equal numbers of sfORNs, imORNs and ffORNs in methionine sensitive cells. The relative high portion of hsORNs in arginine sensitive ORNs $(45 \%)$ could be explained by the relative high portion of sfORNs among them. Assuming sfORNs were more likely to be hsORNs, such a distribution could be expected. Most surprising was the distribution among alanine sensitive cells. Although having the lowest portion of ffORNs (4 out of 15 ORNs) those ORNs had a very high number of lsORNs (14 out of 15 ORNs). The tested ORNs seemed therefore to be less affine to alanine compared to the other tested amino acids as the spontaneous firing rates would have let expect the opposite distribution for alanine sensitive cells. However, the difference in alanine-sensitive ORNs compared to alanine- and methionine-sensitive cells was statistically not significant.

To exclude the second possibility, the ratio of maximum firing rate $f_{\max }$ and averaged (spontaneous) firing rate (aSFR) for different stimulus concentrations was calculated. Importantly, ORN responses to $1 \mu \mathrm{M}$ should be clearly distinguishable from spontaneous firing activity as responses to this concentration threshold would characterize the cell as high-sensitive (hsORN) or low-sensitive (see Figure 33 and Table 8). The ratio was determined as the ratio of maximum firing rate after applying the stimulus in a certain concentration $\left(\mathrm{f}_{\max }(\mathrm{c})\right)$ over the averaged spontaneous firing rate. The median value in hsORNs was almost 2-fold higher than that of 1sORNs. Nevertheless, even in the most unfavorable constellation $\mathrm{f}_{\max }(100 \mu \mathrm{M})$ was approximately 6.5 times higher than the basal rate. This value was just slightly bigger than the smallest ratio for stimuli at $1 \mu \mathrm{M}$, minimum $\mathrm{f}_{\max }(1 \mu \mathrm{M}) / \mathrm{aSFR} \approx 4.7$. On average, $\mathrm{f}_{\max }(1 \mu \mathrm{M})$ was more than 9 times bigger than baseline activity and therefore clearly distinguishable. Figure 13 illustrates a typical response of an ORN to a $1 \mu \mathrm{M}$ stimulus. Furthermore, the biggest signal-to-noise ratio was calculated for a lsORN being at 172.5. Thus, an unfavorable signal-to-noise ratio in lsORNs was unlikely to be the rationale behind the correlation observed between SFR and ORN sensitivity. 


\subsection{Dose-response curves of single ORNs}

Next, I focused on the post-stimulation spiking character of ORNs as information about the odor concentration could be encoded in the spiking rate or the total number of APs following stimulus application.

\subsubsection{Maximum instantaneous post-stimulatory frequencies}

Listing the maximal post-stimulation instantaneous frequencies, i.e. the inverse value of the minimum interspike interval $\tau_{\min }$ (Figure 17), showed that the maximal frequencies varied from minimum $3.19 \mathrm{~Hz}$ to maximum $59.19 \mathrm{~Hz}$, depending on the concentrations of the amino acid solutions (Table 4). On average, the ORNs responded in a range of 9.39 to 26 $\mathrm{Hz}$. Thereby, the maximum post-stimulation firing frequencies were widely overlapping for different concentrations.

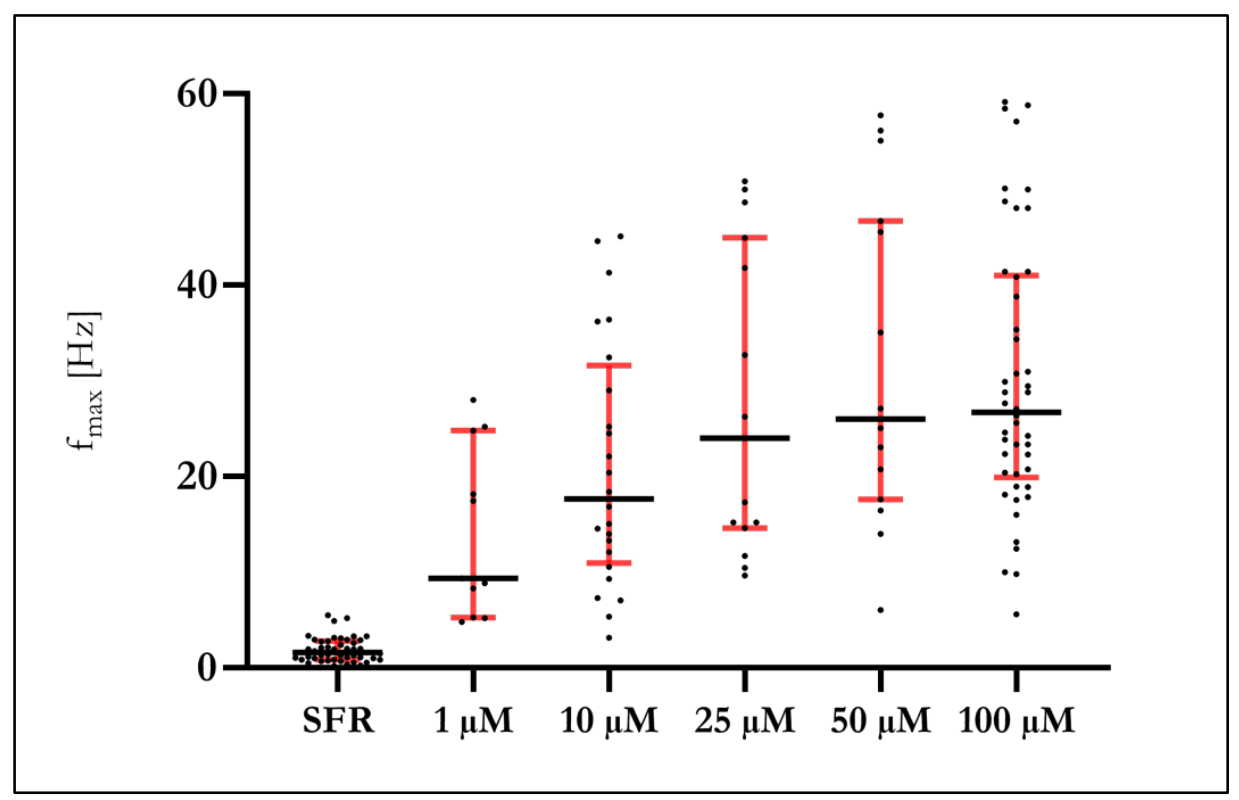

Figure 17 Ranges of maximum instantaneous frequencies

Spontaneous firing rates and maximum instantaneous frequencies (as the reciprocal value of the shortest inter spike interval $\tau$ ) after each stimulus application in 46 ORNs. Bar graphs represent median value with interquartile range (in red). The (stimulated) frequencies varied from 3.19 to $59.17 \mathrm{~Hz}$ among all ORNs and all applied concentrations. The frequencies were widely overlapping for the applied stimuli. The lowest $\mathrm{f}_{\max }(100 \mu \mathrm{M})=5.6 \mathrm{~Hz}$ was thereby approximately 5 times smaller than the maximum $\mathrm{f}_{\max }(1 \mu \mathrm{M})=28.0 \mathrm{~Hz}$. In some ORNs stimulus concentration differences of a 2-log order elicited similar maximum firing rates. Furthermore, the firing frequencies were nearly totally overlapping for "neighboring" concentrations of stimuli. 
The intemeuronal comparison showed that some ORNs responded to single amino acid solutions at $100 \mu \mathrm{M}$ with lower firing frequencies than other ORNs activated by concentrations of $1 \mu \mathrm{M}$ (see Table 4 ; the minimum value for $\mathrm{f}_{\max }(100 \mu \mathrm{M}$ ) was almost 5 times smaller than maximum $\left.\mathrm{f}_{\max }(1 \mu \mathrm{M})\right)$.

Table 4 Averaged instantaneous post-stimulation frequencies

\begin{tabular}{|cccccc|}
\hline $\mathbf{f}_{\max }[\mathrm{Hz}]$ & $\mathbf{1} \boldsymbol{\mu \mathbf { M }}$ & $\mathbf{1 0} \boldsymbol{\mu \mathbf { M }}$ & $\mathbf{2 5} \boldsymbol{\mu \mathbf { M }}$ & $\mathbf{5 0} \boldsymbol{\mu} \mathbf{M}$ & $\mathbf{1 0 0} \boldsymbol{\mu \mathbf { M }}$ \\
\hline $\mathbf{M i n}$ & 4,82 & $\mathbf{3 , 1 9}$ & 9,65 & 6,10 & 5,64 \\
\hline $\mathbf{Q 1}$ & 6,82 & 12,41 & 14,92 & 19,21 & 18,96 \\
\hline Median & 9,39 & 19,45 & 24,03 & 26,01 & 26,00 \\
\hline $\mathbf{Q 3}$ & 21,51 & 31,60 & 43,39 & 46,15 & 39,35 \\
\hline $\mathbf{M a x}$ & 28,01 & 45,15 & 50,89 & 57,80 & 59,17 \\
\hline
\end{tabular}

Thus, these results demonstrated that instantaneous frequencies only informed about stimulus concentration altitude if the maximum firing rate $\mathrm{f}_{\max }(\mathrm{c})$ was high enough (e.g. a frequency $f_{\max }>28 \mathrm{~Hz}$ suggested a corresponding concentration $\left.c>1 \mu \mathrm{M}\right)$. It is not possible to distinguish among the applied concentrations for lower firing frequencies. However, the intraneuronal comparison of $\mathrm{f}_{\max }(\mathrm{c})$ showed that - except of a very few ORNs $-f_{\max }(c 2) \geq f_{\max }(c 1)$, if $c 2>c 1$ (Figure 13). Thus, for a single neuron the spiking rate could provide a tool of concentration encoding. The widely overlapping maximal firing frequencies across all ORNs, however, suggested that ensembles of converging ORNs will not encode the stimulus concentration in spiking rates sufficiently. Especially in low firing ranges, information about the applied concentration could not be coded by the spiking rate only, at least not in a concentration range of $1 \mu \mathrm{M}$ to $100 \mu \mathrm{M}$. As the SFRs ranged from 0.24 to $5.5 \mathrm{AP}$ per second (Table 3 ) the measured low post-stimulation frequencies were already overlapping with the SFRs. Therefore, lower post-stimulation firing frequencies than the above mentioned could not be distinguished from baseline spiking. Furthermore, the spiking frequencies were not encoding the identity of the applied stimulus as low or high firing rates were not specific for any of the applied amino acids (data not shown). Moreover, the SFR and $\mathrm{f}_{\max }$ (c) were only slightly correlated (Figure 18). Slow and fast ORNs do not consistently respond to stimuli with low and high post-stimulation firing frequencies, respectively. Some slow ORNs had a wider dynamic firing range, enabling them to spike in a low frequency range at steady state but also to fire at high rates when being stimulated. The observed maximum firing rates $(\sim 60 \mathrm{~Hz})$ was seen in sfORNs as well as in ffORNs. In most cases it was neither possible to predict the stimulus 
concentration nor the identity of the applied stimulus by only knowing the maximum firing frequency.
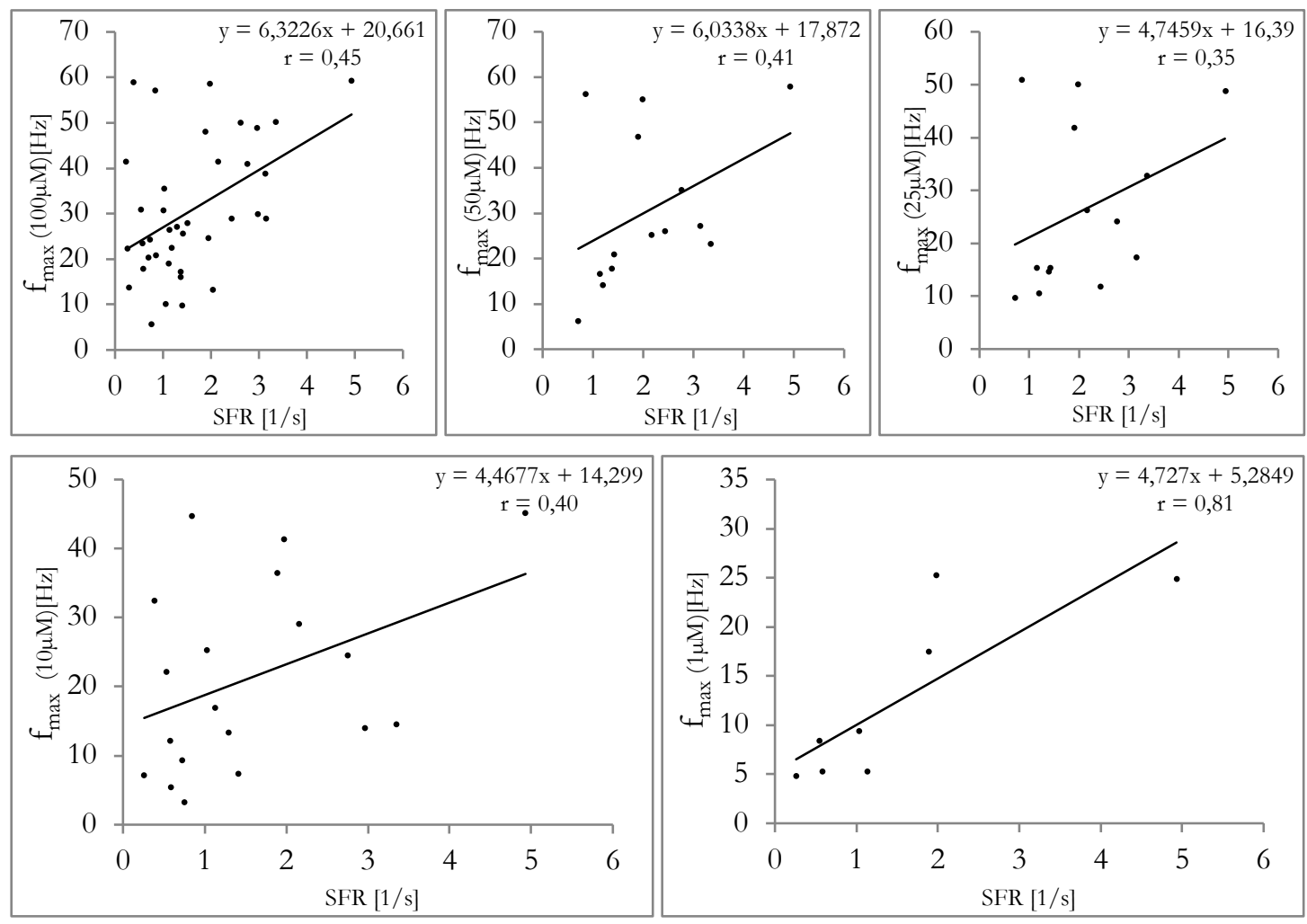

Figure 18 Correlation $\mathrm{f}_{\max }(\mathrm{c})$ and SFR

In $n=36$ ORNs the data points of SFR and $f_{\max }(c)$ were correlated and plotted. Additionally the regression curve was calculated and overlaid to the data points. For all applied concentrations there was a low correlation of SFR and $f_{\max }(c)$ suggesting that sfORNs and ffORNs would not consistently respond in correspondently low and high $\mathrm{f}_{\max }(\mathrm{c})$ post stimulatory, respectively. Slow firing ORNs fired at the highest measured spiking rate as well as fast firing ORNs. For applied concentrations of $1 \mu \mathrm{M} \mathrm{f}_{\max }$ (c) and SFR showed a high correlation coefficient which was probably due to the low number of data points as for all higher applied concentrations, the correlation was very weak (see correlation coefficient $r$ in the right upper part of each subfigure).

\subsubsection{Number of APs as coding pattern?}

If the instantaneous frequency was insufficient to encode odor concentration then could the total number of generated APs provide an alternative mechanism to encode such information?

In the patch-clamp recordings the response of the cell after each stimulus application was terminated by a firing pause of the cell (see Figure 13). During this pause the ORNs did not generate any spontaneous APs but showed a behavior indicating a kind of partly refractory 
state of the ORN after stimulation. The pause was on average approximately 38 times bigger than the averaged inter spike interval of all spikes from stimulus application to the pause (ration of pause duration to averaged spontaneous firing rate: mean \pm 1 standard deviation: $38.33 \pm 24.50 ; \mathrm{n}=6$ ). The size of the pause could not be determined exactly as every pause (at high stimulus concentrations) was interrupted by the application of Ringer's solution. The application of ringer solution elicited a small response after stimulating the OE with high concentrated amino acid solutions, possibly due to amino acids remaining in the funnel tube. Therefore, further analysis on the pauses could not be performed. Yet, in order to set an objective criterion for the start and the termination of a response the observed pause was considered as the end of a response as hereafter the ORNs recovered its SFR. The stimulus application was set as the starting point of the response. It was not possible to decide which spike was actually the first spike of the response and did not belong to baseline spiking anymore. Therefore, the first $1-3$ spikes following for each stimulus application could be categorized as spontaneous baseline firing. Because of the relative high number of the action potentials triggered by the stimuli, I assumed that neglecting 1-3 spikes would not much affect the analysis.

First, the total number of action potentials was counted for each stimulus concentration compared to the spontaneous spikes in the same time range before stimulus application (Figure 19). On average, the highest applied concentration elicited the highest number of spikes from the onset to the termination of a response, with the number of spikes ranging from 13 to 135 for $10 \mathrm{nM}$ and $100 \mu \mathrm{M}$, respectively (Table 5). In a similar time range, before stimulus application, the cell was firing on average 16 spikes, which overlapped with the number of spikes induced by the lowest applied concentration. The time span from the stimulus application to the termination of the ORN response was approximately $4 \mathrm{~s}$ for low-concentrated $(10 \mathrm{nM}$ and $100 \mathrm{nM})$ stimuli, whereas it is around $20 \mathrm{~s}$ for high concentrations. Minimum and maximum values were measured at $2.9 \mathrm{~s}(10 \mathrm{nM})$ and $24.3 \mathrm{~s}$ $(50 \mu \mathrm{M})$, respectively. It was therefore reasonable to calculate the spike numbers with respect to the duration of a response (Figure 19, subfigure B). Before stimulus application the ORNs generated on average 1.2 spikes per second, after stimulation, the average firing

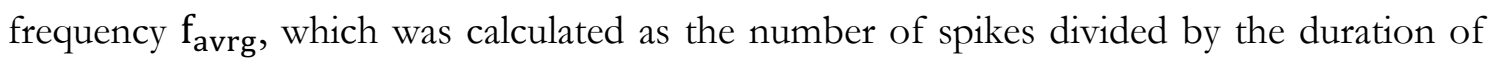
the total response, varied from 3.2 spikes per second $(10 \mathrm{nM})$ to 11.2 spikes per second $(100 \mu \mathrm{M})$ (Figure 19, subfigure B1). 


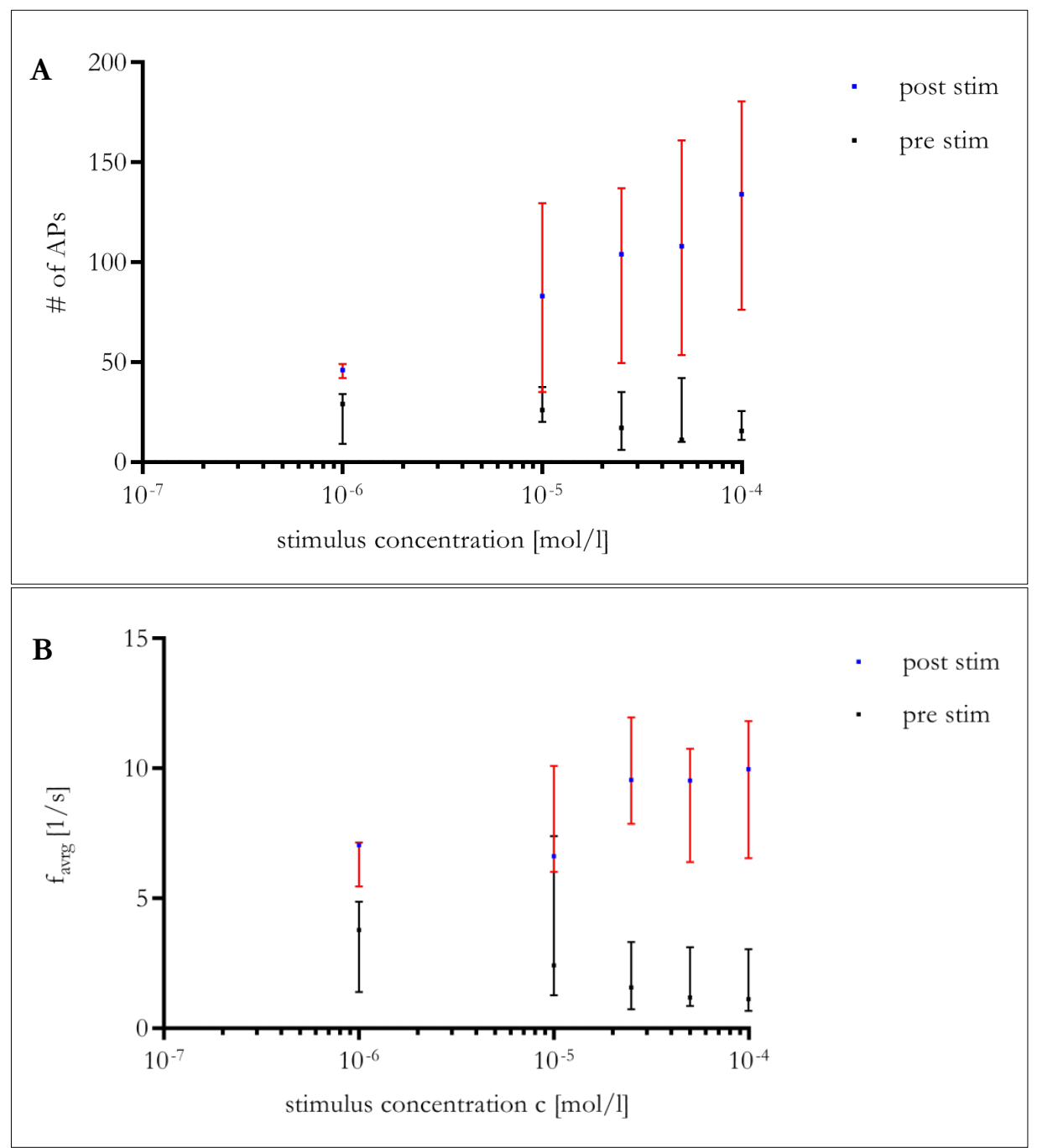

Figure 19 Number of APs and averaged firing rates

A | The box plots represent the number of spikes after stimulus application (blue box is the median value; red error bars represent interquartile range) and before (black box plots; median and interquartile range). For the lowest applied concentration ( $10 \mathrm{nM}, 2$ out of 6 ORNs) 13.5 spikes on average were observed, whereas the ORN fired on average 134 spikes for the highest applied concentration. The total numbers of spikes increased with higher concentrations, however, widely overlapping across the ORNs. B $\mid$ The total number of spikes was divided by the duration of the response. On average a range of 3.2 spikes $(10 \mathrm{nM})$ to 11.1 spikes $(100 \mu \mathrm{M})$ was generated for the response period. $\mathrm{f}_{\text {avrg }}=$ average rate of APs; post stim = mean value after stimulus application; pre stim $=$ mean value before stimulus application; $\mathrm{AP}=$ action potential. 
Table 5 Number of post-stimulation APs

\begin{tabular}{|cccccccc|}
\hline $\begin{array}{c}\text { \# of post- } \\
\text { stim. APs }\end{array}$ & $\mathbf{1 0} \mathrm{nM}$ & $\mathbf{1 0 0} \mathrm{nM}$ & $1 \mu \mathrm{M}$ & $\mathbf{1 0 \mu \mathrm { M }}$ & $\mathbf{2 5} \boldsymbol{\mu M}$ & $\mathbf{5 0} \boldsymbol{\mu M}$ & $\mathbf{1 0 0} \boldsymbol{\mu M}$ \\
\hline Min & 12 & 24 & 42 & 32 & 41 & 30 & 38 \\
\hline Q1 & 12.75 & 24.5 & 44 & 38 & 58 & 77 & 98.25 \\
\hline Median & 13.5 & 25 & 46 & 83 & 104 & 108 & 134 \\
\hline Q3 & 14.25 & 25.5 & 47.5 & 109 & 126 & 149 & 166 \\
\hline Max & 15 & 26 & 49 & 150 & 148 & 173 & 200 \\
\hline
\end{tabular}

Table 6 Averaged rate of post-stimulation APs

\begin{tabular}{|cccccccc|}
\hline $\begin{array}{c}\text { favrg } \\
{[1 / \mathrm{s}]}\end{array}$ & $10 \mathrm{nM}$ & $100 \mathrm{nM}$ & $1 \mu \mathrm{M}$ & $10 \mu \mathrm{M}$ & $25 \mu \mathrm{M}$ & $50 \mu \mathrm{M}$ & $100 \mu \mathrm{M}$ \\
\hline Min & 2.61 & 4.54 & 5.12 & 5.74 & 6.55 & 6.45 & 7.91 \\
\hline $\mathbf{Q 1}$ & 2.91 & 5.34 & 5.85 & 6.62 & 8.96 & 9.53 & 9.27 \\
\hline Median & 3.20 & 6.13 & 6.59 & 9.25 & 10.15 & 9.93 & 11.12 \\
\hline Q3 & 3.49 & 6.93 & 7.47 & 10.01 & 11.24 & 10.33 & 11.27 \\
\hline Max & 3.79 & 7.72 & 8.35 & 11.14 & 12.67 & 11.24 & 13.73 \\
\hline
\end{tabular}

In summary, both the total number of spikes and $f_{\text {avrg }}$ increased with higher concentrations but were highly overlapping across different neurons for different concentrations. Therefore, they were comparable with $f_{\max }$ regarding information encoding at the OE level. It seemed unlikely that the number of spikes or the spike density provided additional information than the instantaneous maximum frequency $\mathrm{f}_{\max }$ did. To test the hypothesis that both the number of APs and maximum instantaneous frequency $\mathrm{f}_{\text {max }}$ carried out the same information, both parameters were correlated to each other over 6 cells and $5-7$ different stimulus concentrations (i.e. $n=40$ repeats) (Figure 20). All data points yielded in a linear correlation with a high correlation coefficient of approximately $\mathrm{r}=0.88$. 


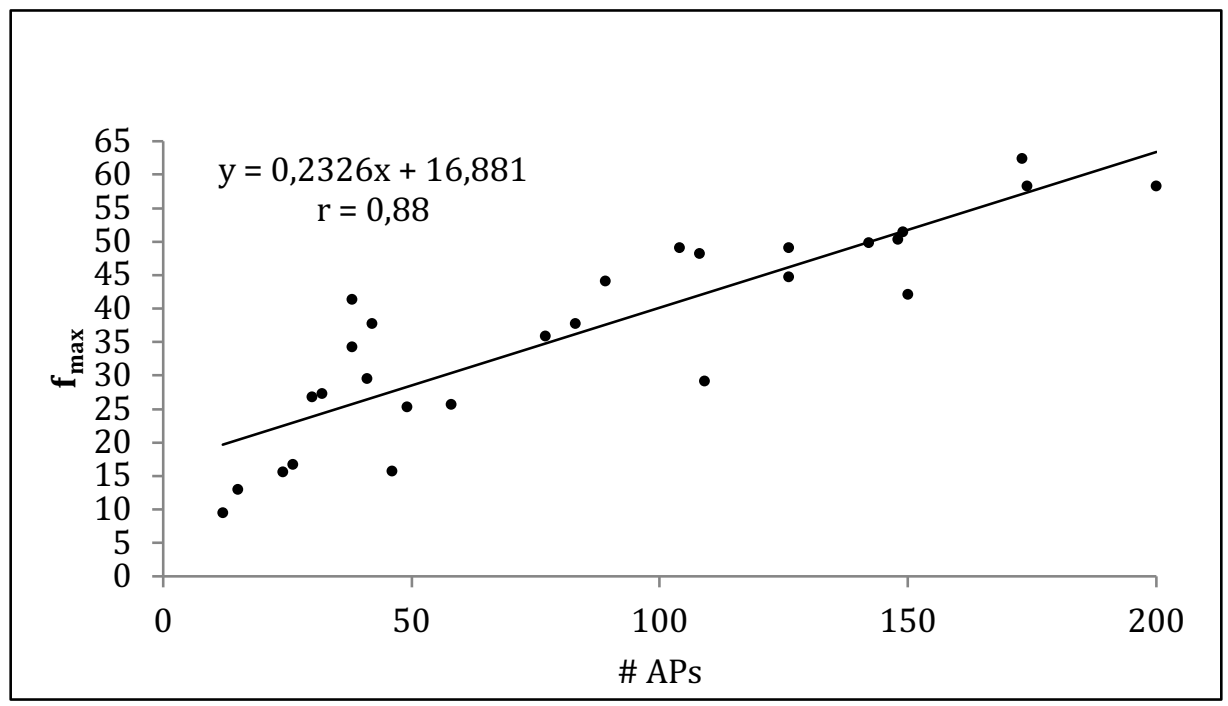

Figure 20 Correlation of $\mathbf{f}_{\max }$ and number of APs

After stimulus application, the number of APs and the maximum instantaneous frequencies were determined in 5 ORNs with 7 different stimulus concentrations and 1 ORN with 5 different stimulus concentrations. Both parameters were highly correlated to each other (correlation coefficient $r=0.88)$.

Finally, one striking observation was made when plotting the dose-response curves; some groups of ORNs seemed to be generating similar AP frequencies after stimulus onset. For instance, 4 receptor neurons responded to a $100 \mu \mathrm{M}$-concentrated stimulus with the maximum frequencies of approximately $\mathrm{f}_{\max } \approx 60 \mathrm{~Hz}$ and 4 other ORNs responded with $\mathrm{f}_{\max } \approx 50 \mathrm{~Hz}$ with no other frequencies measured in between (Figure 21). This impression was supported by the fact that the distribution of $\mathrm{f}_{\max }$ for $100 \mu \mathrm{M}$ stimulus concentrations was not Gaussian (KST, p $=0.014, \alpha=5 \%$ ). Moreover, similar firing frequencies were observed for those neurons after application of lower concentrations as well.

In section 3.3.3 the entire time course of these neurons will be plotted and compared across all neurons. 


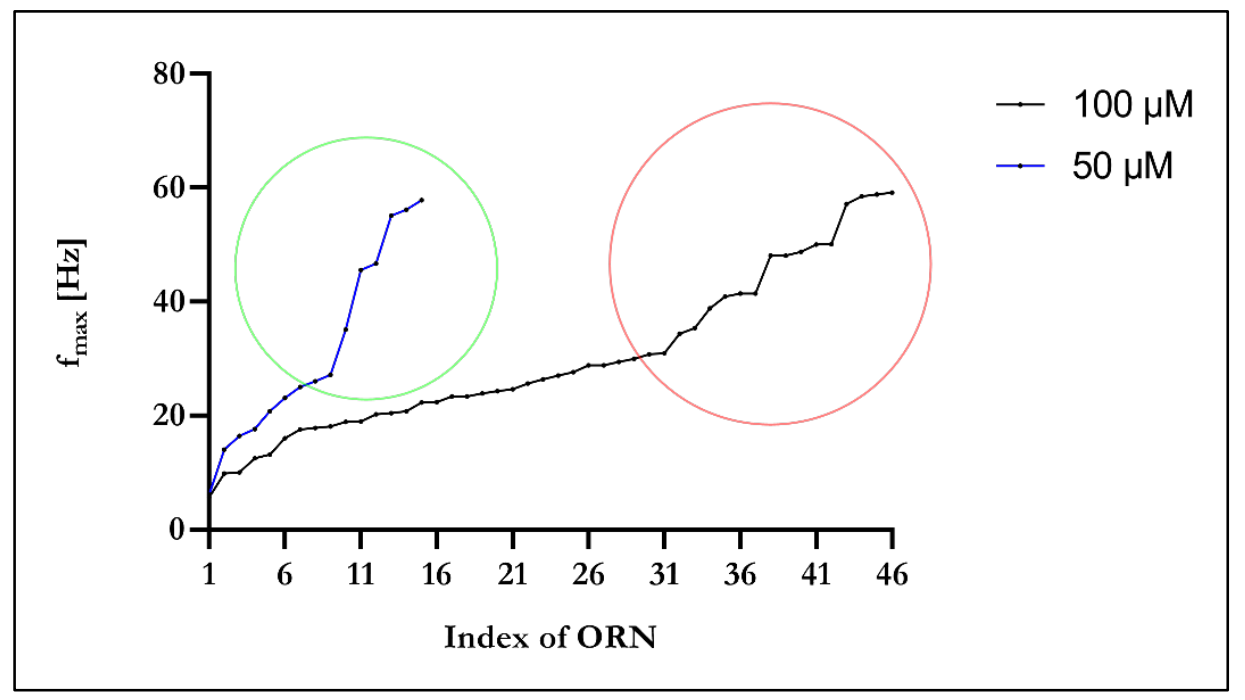

Figure 21 Similarities in response profiles

Sorting the maximum instantaneous frequencies of all ORNs $(n=46)$ from lowest to highest revealed that $\mathrm{f}_{\max }$ increases linearly for low frequencies. For higher frequencies, $\mathrm{f}_{\max }$ in different ORNs seemed to increase gradually. In some ORNs similar frequencies were generated when applying $100 \mu \mathrm{M}$ stimulus concentrations (red encircled). These similarities were echoed in an attenuated manner under lower stimulus concentrations $(50 \mu \mathrm{M}$, green encircled, $\mathrm{n}=15)$. The distribution of $\mathrm{f}_{\max }$ for $100 \mu \mathrm{M}$ is not Gaussian (KST, $\mathrm{p}=0.014, \alpha=5 \%$ ). $\mathrm{f}_{\max }=$ maximum instantaneous frequency after stimulus onset. 


\subsection{Responses of ORN ensembles}

\subsubsection{ORNs' cumulative responses}

In the previous sections, I described that spiking frequencies as wells as number of generated APs in spike trains of single receptor neurons were insufficient to encode information about stimulus concentrations. Possibly, convergence of receptor neuron activities were more suited to encode such information.

For this purpose, firstly, the cumulative responses of 9 selected ORNs to different concentrations were plotted against time (Figure 22). As expected, the number of action potentials per bin (here $500 \mathrm{~ms}$ ), the number of activated neurons and the response duration increased with increasing stimulus concentration. Thereby, application of $100 \mu \mathrm{M}$ concentrated stimulus solution elicited responses with the highest firing rates and the longest durations (ca. 15 s, Figure 22, subfigure A). Although for single ORNs it was not possible to predict a stimulus by the averaged or maximum post-stimulation frequency (section 3.2), ensembles of neurons generated more robust concentration-dependent firing rates that were indicative for the applied stimulus concentration (compare number of spikes/s for different concentrations: approximately 180/s, 130/s, 60/s, 30/s for $100 \mu \mathrm{M}$, $10 \mu \mathrm{M}, 1 \mu \mathrm{M}$ and concentrations in the nanomolar range, respectively). Furthermore, responses to near-threshold concentrations that were hardly distinguishable from background spiking (in single ORN spike trains) became more evident in the cumulative response (see responses to $10 \mathrm{nM}$, E.1). However, this was only the case if at least some ORNs of the ensemble already showed a (doubtful) response in the raw spiking train. In other cases, summation of the responses across neurons did not reveal further response patterns (subfigure E.2). And finally, response termination to high concentrations seemed to follow more complex kinetics than in the case of low concentrations. The decay of the firing rates in highly stimulated ORNs seemed to consist of 2 distinct phases (the presumed transition point is marked by a black star, A and B in Figure 22); a first fast decay followed by second slower decay. In order to verify this observation, the decay of the instantaneous frequencies instead of firing rates in fixed (relatively) large bins are studied in section 3.3.3. 


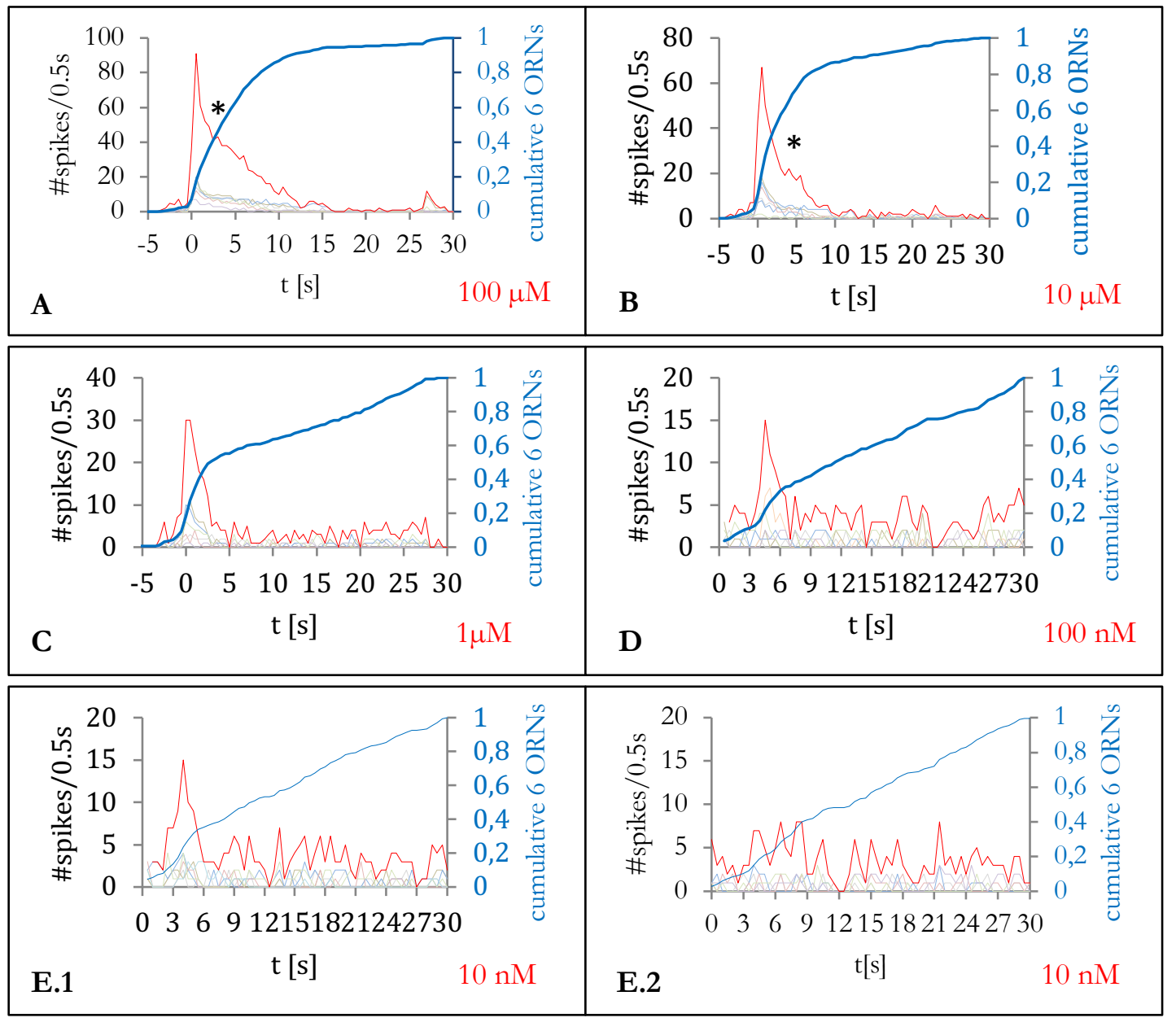

Figure 22 Cumulative responses of 9 ORNs to different stimulus concentrations

Response profiles of 9 different ORNs. All ORNs were highly sensitive ORNs but displayed mixed spontaneous firing rates, i.e. these 9 cells consisted of slow, intermediate and fast firing ORNs. In each subfigure the number of action potentials per bin of $500 \mathrm{~ms}$ is plotted against time on the horizontal axis. The single ORN responses are plotted with desaturated colors in the background whereas the cumulative responses are represented by the red curve being the result of summation of all APs per bin. The blue ascending curves represent the empirical cumulative fraction for the time range displayed. The maximum firing rates for concentrations in the $\mu \mathrm{M}$-range $(\mathrm{A}-\mathrm{C})$ were centered to 0 in order to compare the responses more reasonably because of the trial-to-trail variance of the peak-frequency onset. It was not possible to do this step for stimulations with very low concentrations in the nM-range (D-E) as the maximum firing rate was barely distinguishable from background spiking. Therefore, the time courses in the nM-range cover the first spike after stimulus application to $30 \mathrm{~s}$ post-stimulus application. In section 3.1.2, a firing pause especially after high concentrated stimulus application was described but is neglected here. Each subfigure represents a time course of the response profiles to different concentrations: $100 \mu \mathrm{M}, 10 \mu \mathrm{M}, 1 \mu \mathrm{M}$, $100 \mathrm{nM}$ and $10 \mathrm{nM}$ (subfigures A, B, C, D, E.1). Subfigure E.2 represents the cumulative responses of 6 highly sensitive ORNs that apparently did not respond to concentrations in the nanomolar range by eye-sight. 


\subsubsection{What is characteristic for steady state?}

The data showed so far that changes in frequency or firing rate, i.e. number of APs per bin, could be very discrete after application of stimuli in the nanomolar range. Therefore, it was often impossible to distinguish a stimulus-induced response from the variance of cellular activity in the steady state. It was desirable to develop criteria that enabled to distinguish stationarity from non-stationarity at threshold concentrations. Finding a mathematical model for spontaneous activity could help to predict how many additional (or less in case of inhibitory responses) APs were needed for the olfactory system to recognize a stimulus as such. In section 3.1.1 I reported that spontaneous firing rates of ORNs and multiple inputs of theirs (to a virtual glomerulus) had a Poisson character. Moreover, the cumulative responses revealed that unclear responses at single cell level apparently became more evident when summing up the input of multiple receptor neurons (Figure 22). Consequently, the Poisson distribution of the SFRs of neuronal groups could be used as a definition of the steady state (where event rate $\lambda$ is constant); every deviation from the event rate $\lambda$ (within a certain probability) would represent non-stationarity. To test this model the data of 6 ORNs (from Figure 22 A-E.1) were added and the mean event rate $\lambda$ was calculated after stimulus application (Figure 23). For high concentrations, it was obvious that the mean event rate $\lambda$ of the theoretical Poisson distribution was unequivocally deviant from the observed data (after stimulus onset). After the application of a $1 \mu \mathrm{M}$-concentrated stimulus, the observed distribution resembled more the expected Poisson distribution despite a statistically high significant difference. With this approach, however, the concentrations of interest in the nanomolar range - which showed a clear response (Figure 22, subfigure E.1) - were statistically not distinguishable from the theoretical Poisson distribution (Figure 24). This discrepancy could be expected because of the bin size (=500 ms). The 30 s-long recording time yielded 60 data points of which only 5 - 6 data points contributed to a possible response. To circumvent this problem, I decided to compare the time period directly after stimulus application (first $10 \mathrm{~s}$ after stimulus application) with the second part (following 20s) as stimulus induced activity and recovery to SFR was expected to be $<10 \mathrm{~s}$ for low stimulus concentrations (see 3.2.2). 

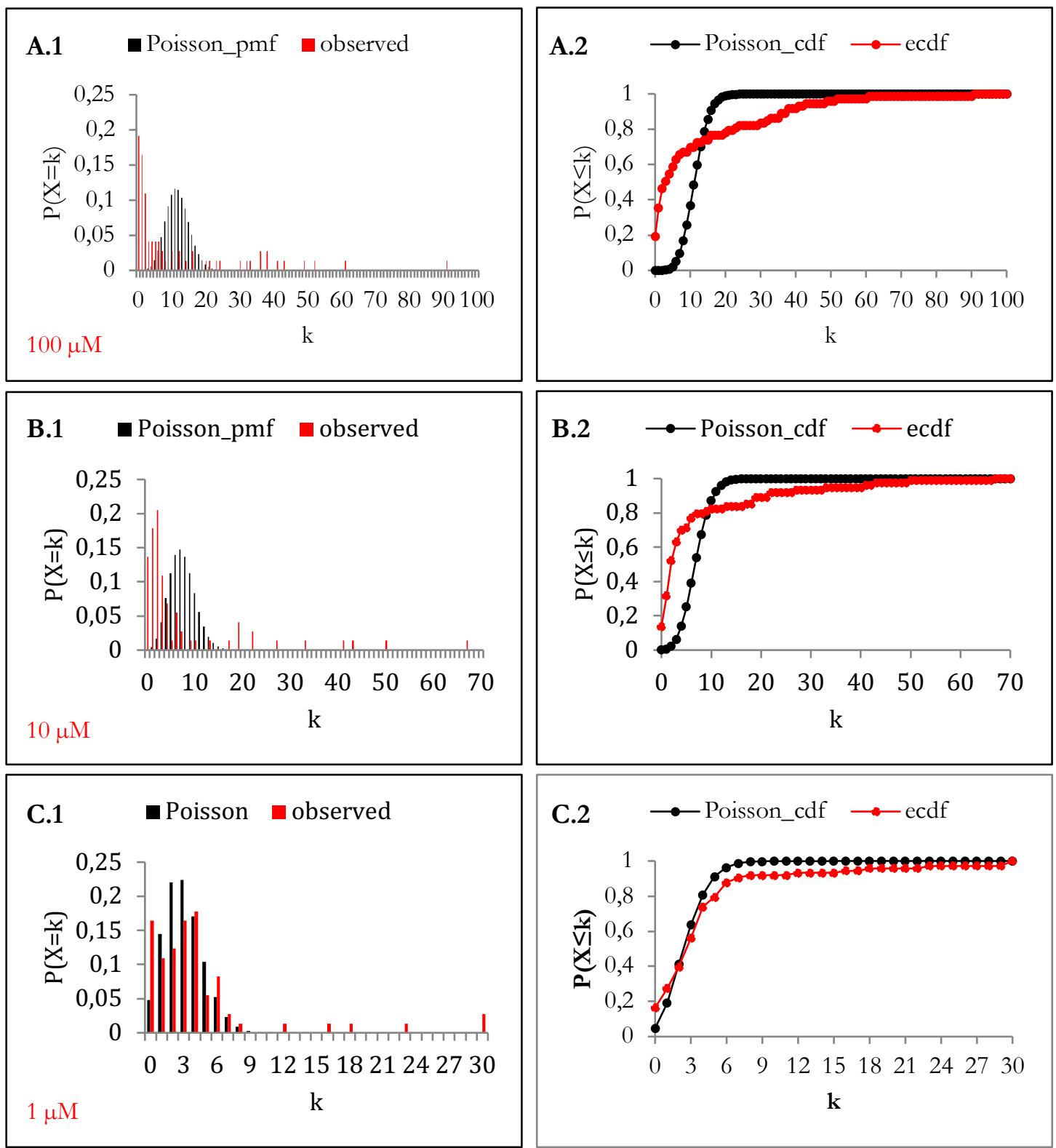

Figure 23 Non-stationarity at high stimulus concentrations

Expected Poisson distribution with mean event rate $\lambda$ (= number of APs per 500 ms-bin averaged for 6 ORNs, 30 s-long recording time) compared to observed data after stimulation. A, B and C display responses to $100 \mu \mathrm{M}, 10 \mu \mathrm{M}$ and $1 \mu \mathrm{M}$ stimulus applications, respectively. The probability mass functions (A.1, B.1 and C.1) already indicate a significant difference of theoretical distributions and observed data. The cumulative distribution functions confirm the statistical significance $(\mathrm{KST}, \alpha=1 \%)$. Pmf $=$ probability mass function, $(\mathrm{e}) \mathrm{cdf}=($ empiricial $)$ cumulative distribution function. 


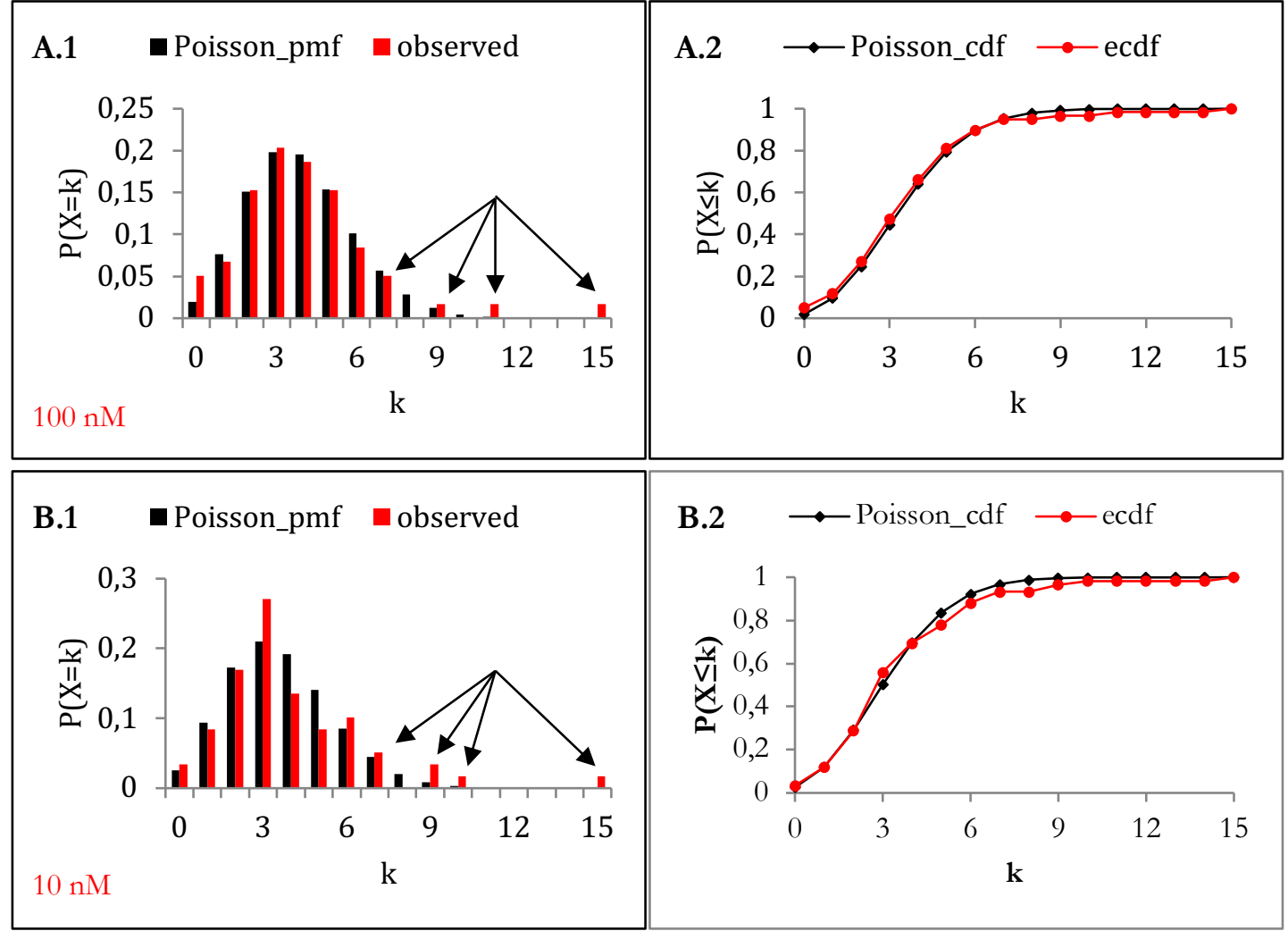

Figure 24 Non-stationarity at low stimulus concentrations

Theoretical Poisson distribution of 6 different ORNs (event rate $\lambda$ is estimated from averaged SFRs) compared to empirical data after stimulus application in the nanomolar range. No statistical difference in the theoretical Poisson distribution and the observed data (APs per $500 \mathrm{~ms}$-bin, $30 \mathrm{~s}$ long recording time). The black arrows indicate the presumed stimulus-induced high frequent AP rates for $100 \mathrm{nM}$ (A.1) and $10 \mathrm{nM}$-concentrated stimuli (B.1).

In steady state both distributions should be similar (assuming a constant event rate $\lambda$ ). Indeed, this approach unambiguously demonstrated that both distributions were different despite the application of threshold concentrations (Figure 25). Moreover, even for the 6 ORNs which apparently did not respond to $10 \mathrm{nM}$ (Figure 22, Subfigure E.2) the comparison of first $10 \mathrm{~s}$ to the subsequent $20 \mathrm{~s}$ showed clearly 2 different distributions (Figure 25, Subfigure C1 and C2) which was statistically significant (KST, $\alpha=1 \%$ ). 


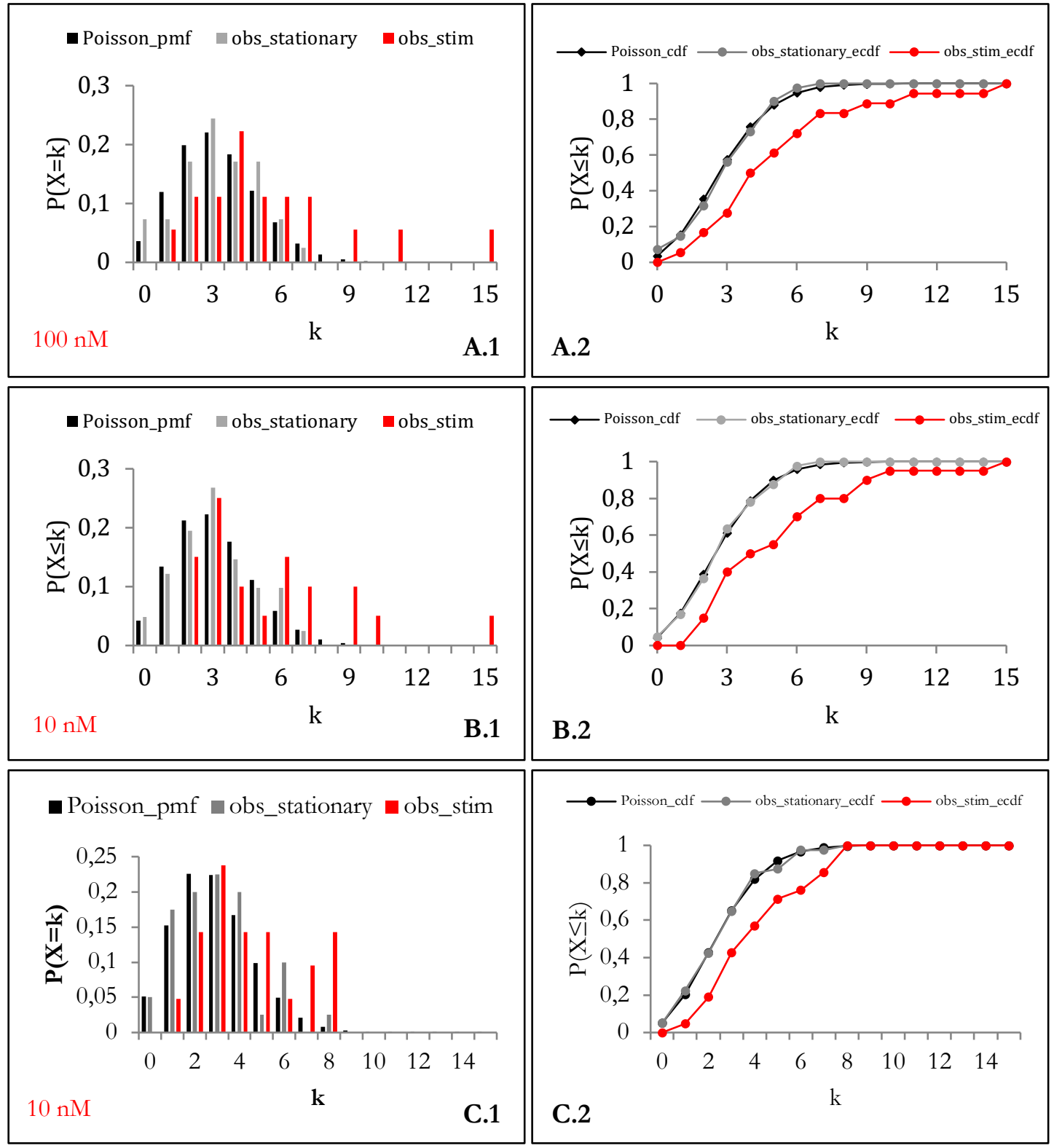

Figure 25 Non-stationarity at threshold concentrations

$\mathrm{A}$ and $\mathrm{B} \mid$ The data of 6 ORNs (identical to those in Figure 24) of the first $10 \mathrm{~s}$ after stimulus application (red) were compared to the following $20 \mathrm{~s}$ (grey) (number of APs per $500 \mathrm{~ms}$-bin, $30 \mathrm{~s}$ long recording time, event rate $\lambda$ estimated by SFRs $10-20 \mathrm{~s}$ after stimulus onset). The data of the first $10 \mathrm{~s}$ were significantly different than the observed distribution of the following $20 \mathrm{~s}$ and the theoretical Poisson distribution after application of $100 \mathrm{nM}$ and $10 \mathrm{nM}$ concentrations (KST, $\alpha=$ $1 \%$ ). C| Even in case of the 6 ORNs that apparently did not respond to $10 \mathrm{nM}$ stimulus concentrations (Figure 22, subfigure E.2) the distribution $10 \mathrm{~s}$ after stimulus application was significantly different than the AP rate $10-30$ s after stimulus application. Poisson_pmf = probability mass function of the Poisson distribution, obs_stim $=$ data of the first $10 \mathrm{~s}$ with presumed stimulus-induced response, obs_stationary $=$ data of last $20 \mathrm{~s}$ of recording time. 


\subsubsection{ORN response profiles and kinetics}

As described at the end of section 3.2.2 some ORNs had apparently similar response profiles, i.e. similar maximum instantaneous frequencies following the same (high) stimulus concentration (Figure 21). It was therefore interesting not only to compare the maximum instantaneous frequencies but the whole time course of the receptor neurons' responses to different stimulus concentrations. Did these similarities exist in the duration of ORN responses? Indeed, the time course of the instantaneous frequencies of $6 / 9$ ORNs revealed remarkable similarities in the response profiles (Figure 26). Notably, these ORNs originated from different animals. Receptor neurons responding with a certain maximum instantaneous frequency (e.g. ORNs 1-3 and ORNs 4-6 in Figure 26) tended to have similar response profiles, even when stimulated at different stimulus concentrations. However, these apparent similarities were only observed in small groups of neurons (6/46 ORNs). Comparing ORN7 to ORNs 1-3 shows different response profiles despite similar maximum instantaneous frequencies $(\sim 60 \mathrm{~Hz})$. Although the comparison between ORNs 1-3 and ORNs 4-6 suggest that ORNs with higher maximum instantaneous frequencies $\mathrm{f}_{\max }$ are high-sensitive ORNs (i.e. responsive to $1 \mu \mathrm{M}$ ) such a conclusion is, however, not valid for all ORNs, as the sensitivity of a receptor neuron only correlates with the SFRs (section 3.1.3). ORN9 was chosen as an example to demonstrate that ORNs with a very low $\mathrm{f}_{\max }$ can be high-sensitive as well (i.e. reactive to $1 \mu \mathrm{M}$ stimulus concentration). Taken together, the exemplary 9 ORNs show that groups of ORNs seemed to have astonishingly high similarities in their response profiles across different animals and stimuli. The response profile, however, could not be predicted by knowing the maximum instantaneous frequency of the cell.

Some of the ORNs appear to show an interesting phenomenon in their response kinetics independently of their response profiles and the concentration of the applied stimulus. For application of high stimulus concentrations (and thus eliciting high spiking rates) the decay of the firing frequency seemed to be biphasic (Figure 26, ORN1-ORN3 $100 \mu \mathrm{M}$ stimulus application). ORN1 - 3 (and ORN7) as well as ORN4 - 6 were identical with the ORNs in Figure 21 (red encircled). A biphasic decay was unexpected as the experimental set up (see Materials and methods) allowed the application of a stimulus without interrupting the slice perfusion with Ringer's solution. Thus, it could be expected that the stimulus concentration decay will be exponential (as a change of concentration over time would be proportional to the actual concentration). And therefore, the decay rate should be constant. 

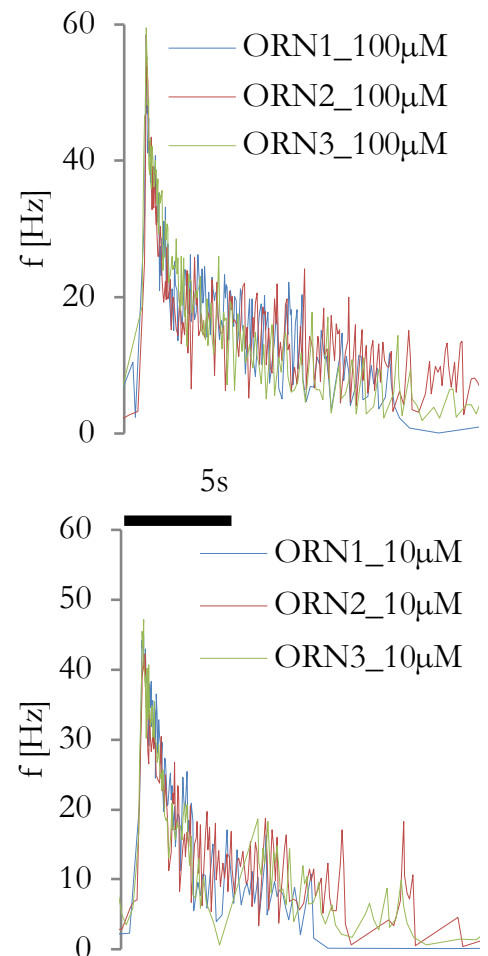

$5 \mathrm{~s}$

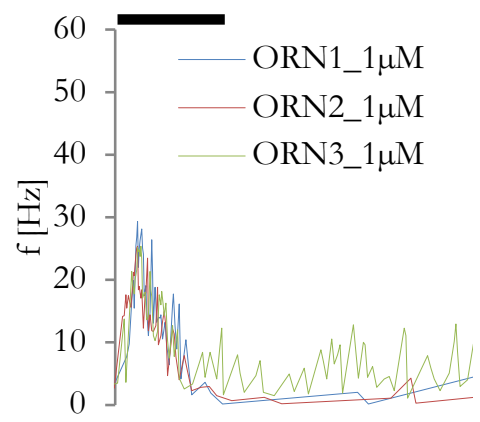

$5 s$
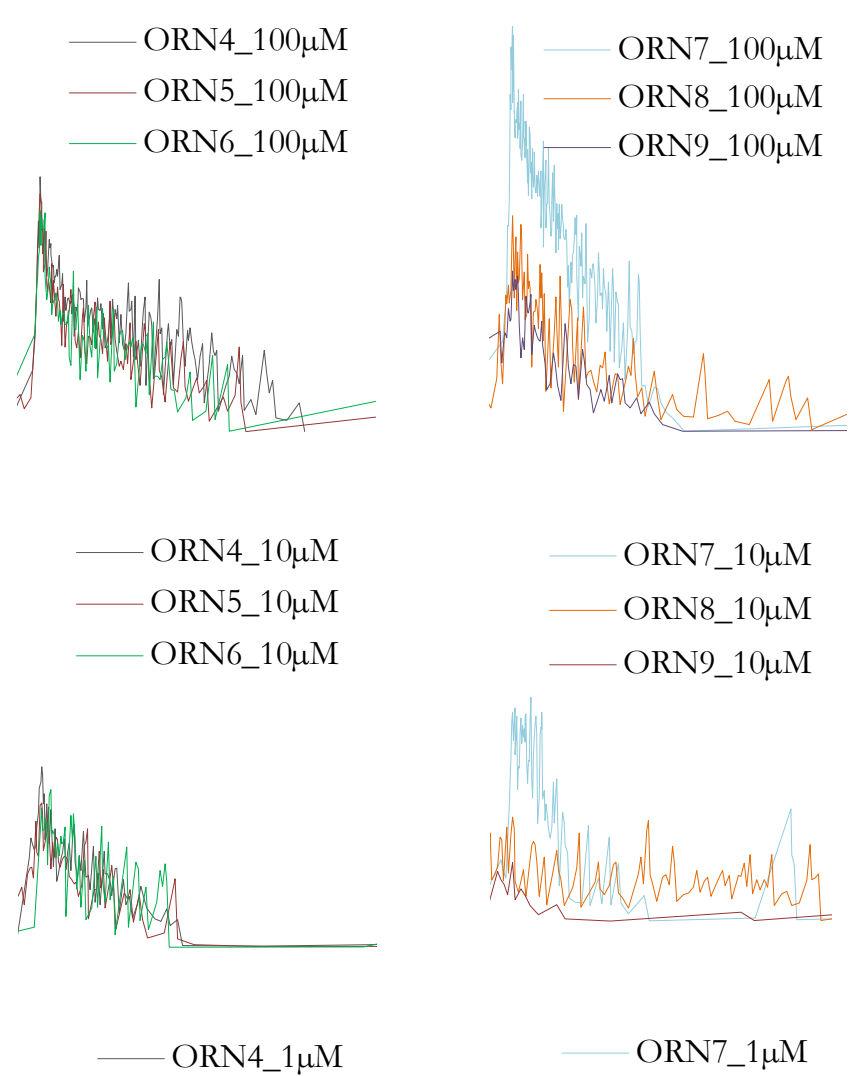

ORN5_1 $1 \mu \mathrm{M}$

ORN6_1 $1 \mu \mathrm{M}$

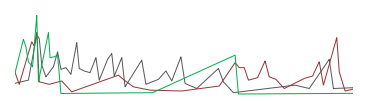

Figure 26 Stimulus-induced instantaneous frequencies over time

Time course of stimulus-induced instantaneous frequencies of 9 ORNs from 9 different tadpoles. The time course covered $16 \mathrm{~s}$, thereby, the time of maximum instantaneous frequency $\left(\mathrm{f}_{\max }\right)(\mathrm{bin}=$ interspike interval) is centered to $t_{0}=0 \mathrm{~s}$ in order to make the time courses comparable. The time course starts $1 \mathrm{~s}$ before $\mathrm{f}_{\max }$-onset. The upper figures represent responses to stimuli with the maximal concentration $(100 \mu \mathrm{M})$, the middle line, responses to $10 \mu \mathrm{M}$ and the bottom line, to 1 $\mu \mathrm{M}$. Remarkable similarities existed in response profiles in different ORNs of different tadpoles over different concentrations (ORNs 1-3 and ORNs 4-6). The stereotypic responses suggested that response profiles depended on the maximal instantaneous firing rates. However, such stereotypic responses were seen only for small groups of neurons. Other ORNs responding with the same maximum frequency (e.g. comparing ORN7 to ORNs 1-3) showed different responses to lower concentrations. Therefore, maximum instantaneous frequency did not determine a stereotypic response and the response threshold of a neuron as response profiles of ORN1-3 would suggest. 
If this was true, then the frequency decay should also be a monophasic exponential decay (in case of a linear relation between stimulus concentration and instantaneous frequency). To test the hypothesis that in some receptor neurons the frequency decay (i.e. recovery to steady state) was a biphasic process the empirical data obtained from ORN1-9 (identical to the neurons in Figure 26) after application of the highest stimulus concentration $(100 \mu \mathrm{M})$ were fitted to 2 exponential functions with the least-squares regression: the simpler of both functions (with 1 decay rate $\mathrm{k}$ ) was thereby nested in the more complex function (with 2 decay rates $\kappa_{\text {fast }}$ and $\left.\kappa_{\text {slow }}\right)$. To decide whether the mono- or biphasic decay model was more suited to fit the empirical data the extra sum-of-squares F-test was performed. The monophasic decay model tended to underestimate the empirical data at high frequencies and overestimated the data at low frequencies when stimulating with high stimulus concentrations, respectively (Figure 27).
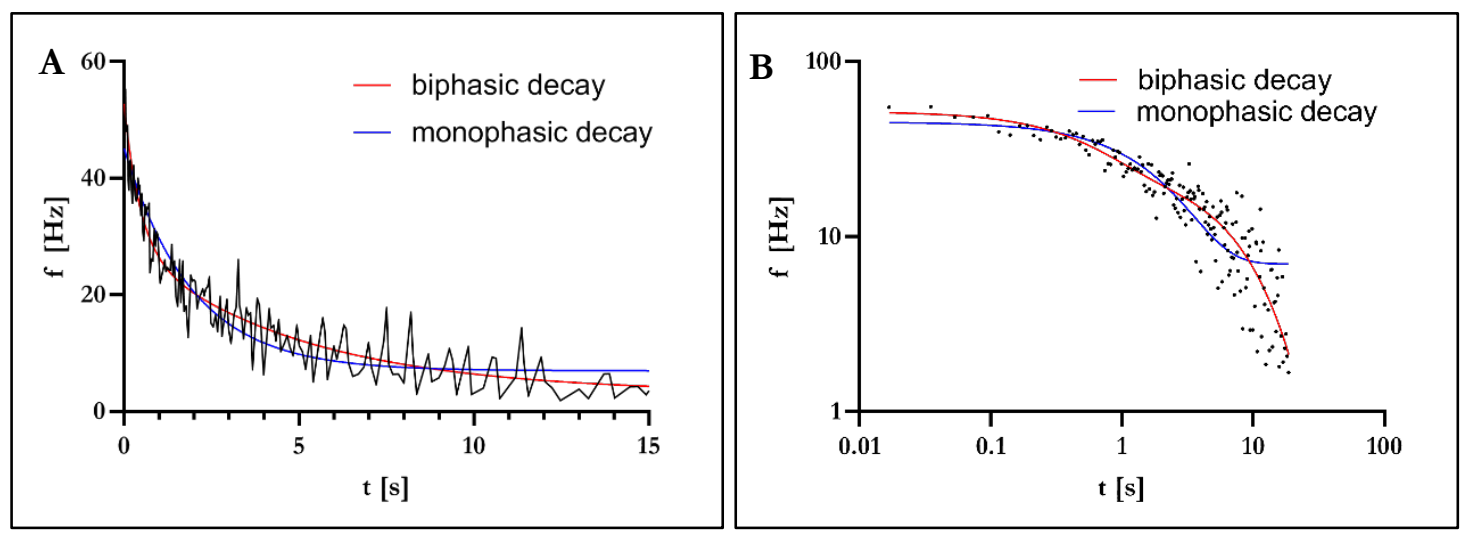

Figure 27 Biphasic Recovery

A Instantaneous frequency $\mathrm{f}$ (y-axis) decay over time of an exemplary ORN (identical with ORN3 in Figure 26) after high-concentrated stimulus application $(100 \mu \mathrm{M})$. At $t_{0}=0 \mathrm{~s}$ frequency is maximal $\left(f_{\max }\right)$. The empirical data are fitted with a monophasic (blue graph) and a biphasic (red graph) exponential function (time range: $t_{0}$ up to $t_{p}$ before onset of firing pause (here $t_{p}=18.7 \mathrm{~s}$ )). B | Logarithmic scaling of the axes (data identical to A) reveal underestimation of the empirical data at high frequencies and an overestimation at low frequencies in the monophasic model. Thus, the biphasic decay model was favored over the monophasic model for this ORN (extra sum-of-squares F-test, $\mathrm{F}=38.5, \mathrm{p}<0.0001)$.

Monophasic exponential function: $\mathrm{f}_{1}(\mathrm{t})=38.2 \mathrm{~Hz} * \mathrm{e}^{-0.52 \mathrm{t}}$ and biphasic exponential function: $\mathrm{f}_{2}(\mathrm{t})=27.5 \mathrm{~Hz} * \mathrm{e}^{2.26 \mathrm{t}}+25.3 \mathrm{~Hz} * \mathrm{e}^{-0.21 \mathrm{t}} ; \mathrm{f}=$ instantaneous frequency, $\mathrm{t}=$ time in seconds. 
In $7 / 9$ ORNs of the tested receptor neurons (ORN1 - 7) the biphasic model was the more adequate fitting the empirical data when the receptor neurons were stimulated at saturating concentration $(100 \mu \mathrm{M})$. ORN1 - 7, thereby, exhibited the highest frequencies in response to stimulus application. In contrast, ORN8 - 9 had lower frequencies and the monophasic exponential decay was favored in these neurons (Table 7). However, $\mathrm{f}_{\text {max }}$ value did not explain entirely why the decay in some receptor neurons was biphasic and in some ORNs not; e.g. ORN8 and ORN6 had similar $\mathrm{f}_{\max }$ but in ORN8 the monophasic model was favored. Moreover, when testing for the same neurons at lower stimulus concentrations (10 $\mu \mathrm{M})$ the biphasic model was favored only in ORN2 - 4 but not in ORN1 and ORN5-9, respectively.

Table 7 Biphasic decay

\begin{tabular}{|lllllllllll|} 
& ORN1 & ORN2 & ORN3 & ORN4 & ORN5 & ORN6 & ORN7 & ORN8 & ORN9 \\
\hline $\mathbf{f}_{\text {max }}[\mathrm{Hz}]$ & 58.5 & 58.5 & 59.5 & 37.3 & 34.7 & 32.4 & 61.0 & 32.1 & 23.9 \\
\hline $\boldsymbol{\kappa}_{\text {fast }}\left[\boldsymbol{s}^{-\mathbf{1}}\right]$ & 2.64 & 2.67 & 2.25 & 3.42 & 4.61 & 3.14 & 16.4 & -- & -- \\
\hline $\mathbf{\kappa}_{\text {slow }}\left[\boldsymbol{s}^{-\mathbf{1}}\right]$ & 0.1 & 0.06 & 0.21 & 0.1 & 0.14 & 0.12 & 0.12 & -- & -- \\
\hline $\mathbf{K}$ - ratio & 25.9 & 42.8 & 10.9 & 33.8 & 32.6 & 25.0 & 138 & -- & -- \\
\hline $\mathbf{F}$ & 79.5 & 88.8 & 38.5 & 38.8 & 34.4 & 22.14 & 6.78 & 0.20 & 2.00 \\
\hline $\mathbf{p}$-value & $<0.0001$ & $<0.0001$ & $<0.0001$ & $<0.0001$ & $<0.0001$ & $<0.0001$ & 0.0015 & 0.82 & 0.17 \\
\hline
\end{tabular}

For ORN1-9 maximal instantaneous frequencies (in response to $100 \mu \mathrm{M}$ stimulus concentration) are displayed. In ORN1-7 the biphasic decay model is favored over the monophasic decay as evaluated with the extra sum-of-squares F-test. The F-statistic is the ratio of relative sum-of-squares to the relative degrees of freedom in biphasic model compared to monophasic model $F=\frac{\frac{S S 1-S S 2}{S S 2}}{\frac{D F 1-D F 2}{D F 2}}$ where SS1 = sum of squares in monophasic model, SS2 = sum of squares in biphasic model, DF is degree of freedom in mono - (DF1) and biphasic (DF2) model. If biphasic model was the favored model $\kappa_{\text {fast }}$ and $\kappa_{\text {slow }}$ correspond to the decay rates in the biphasic model. Notably, on average $\kappa_{\text {fast }}$ is approximately 44 times bigger than $\kappa_{\text {slow }}$. 


\subsubsection{Distribution of the length of interspike interval $\tau$}

Unlike during the steady state, the numbers of action potentials in a certain time range are expectably not constant in stimulated state. Nonetheless, despite changes in the rate of APs, the length of interspike intervals may have Poisson character in the stimulated state as well. If this was the case then would both the short and long interspike intervals have Poisson character? In steady state in some bursting neurons, sequences of short interspike intervals did not have Poissonian character (see 3.1.1).

To decide if the interspike intervals in the stimulated state were Poisson-distributed the same way of analysis as used in steady state (Figure 9) was performed, i.e. all interspike intervals were arranged from shortest to longest and categorized in $1 \mathrm{~ms}$-long bins. In order to compare short $\tau \mathrm{s}$ with longer $\tau \mathrm{s}$ the data points were divided into 4 equally sized interval length ranges (ranges were chosen arbitrary). This approach was demonstrated in a single ORN (Figure 28). Hereby, the first interspike interval range covered all $\tau$ s from 18 ms to $100 \mathrm{~ms}$ of length; all following ranges had the same range length, i.e. $83 \mathrm{~ms}$. In that cell, the distribution of $\tau$ s was homogenous in the longer length ranges only. Thus, the distribution of the shortest $\tau \mathbf{S}(18-100 \mathrm{~ms})$ is not a Poisson distribution. Even though the second and third interval range (101-183 ms and 184-266 ms) were statistically not different than a Poisson process they were still not perfectly Poisson-distributed. Since the cut off value of $100 \mathrm{~ms}$ was set artificially it could not excluded that longer interspike intervals had a non-Poisson character as well. Poisson character means here that in a given interval range the interspike intervals were "scattered" homogenously over the range. To verify this finding in all ORNs, the cumulative data of all 9 ORNs (from Figure 26) were analyzed in the same manner. Thereby, the $\tau$ s of ORNs 1-3, ORNs 4-6 and ORNs 7-9 were pooled and categorized into 1 ms-bins. By analogy with the interspike interval ranges of the single cell (see above) the cumulative intervals were divided into 4 groups with interspike interval ranges of equal size (Figure 29). Remarkably, the data of all 9 ORNs in response to 3 different concentrations $(1 \mu \mathrm{M}, 10 \mu \mathrm{M}$ and $100 \mu \mathrm{M})$ for 4 different lengths ranges yielded $9 * 3 * 4=108$ data points, which confirmed that only the shortest interspike intervals were not Poisson-distributed whereas interspike intervals $\tau>100 \mathrm{~ms}$ were. That was indicative for a non-homogeneous distribution of short interspike intervals as with this approach, the observed data were deviant from the theoretical event rate $\lambda$ which was the average number of $\tau$ s per 1 ms-bin. 


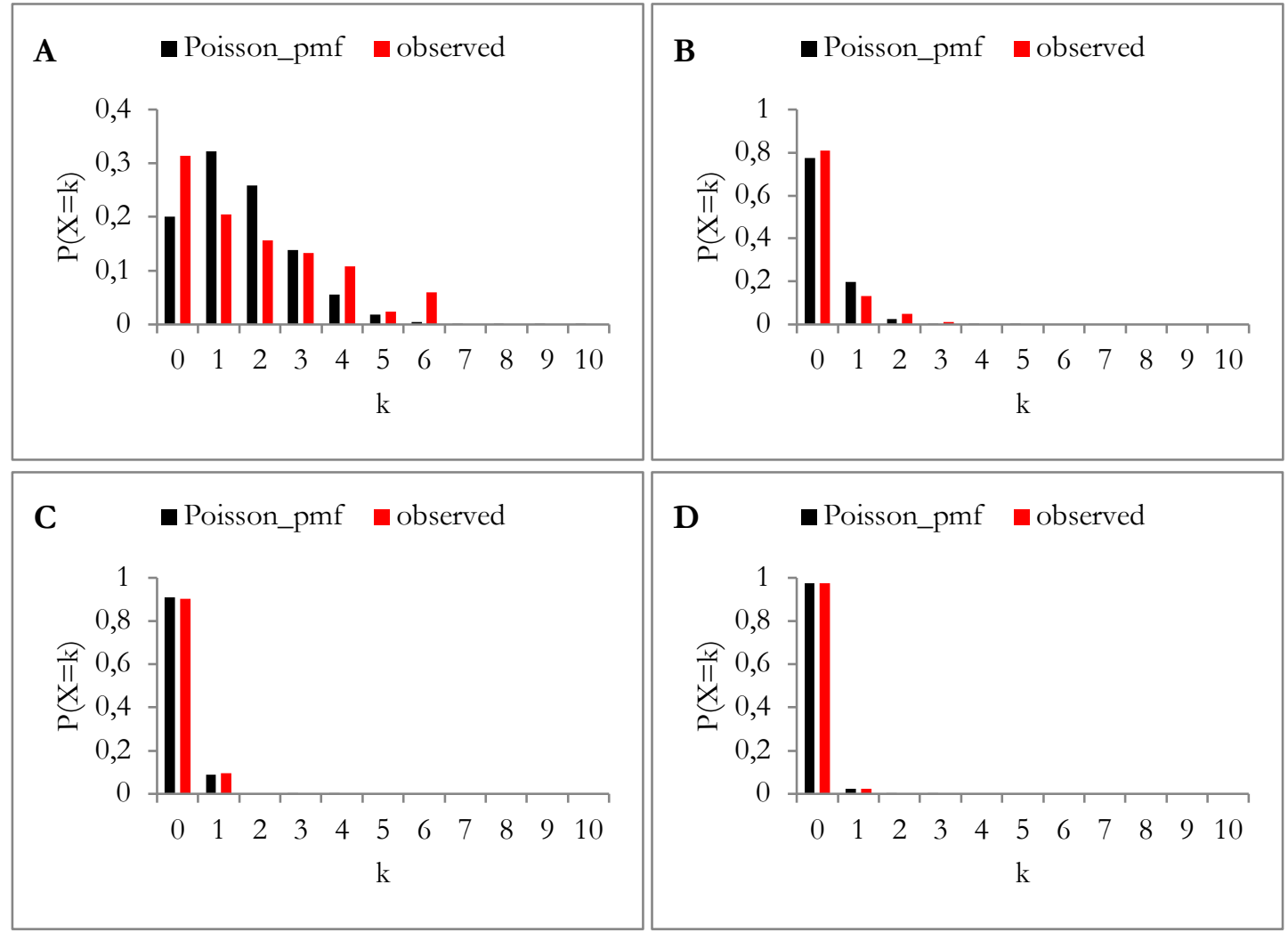

Figure 28 Stimulus-induced patterns of interspike intervals in a single ORN

All interspike intervals $\tau \leq 350 \mathrm{~ms}(\approx 98 \%$ of all data) were categorized in $1 \mathrm{~ms}$-bin such that short $\tau$ S $(\tau<100 \mathrm{~ms}, \mathrm{~A})$ were compared to longer $\tau$. Thereby, the length range of the shortest interspike intervals $(18-100 \mathrm{~ms})$ was equally sized to longer interval length ranges $(101-183 \mathrm{~ms}$, B; $184-266 \mathrm{~ms}$, C and $267-350 \mathrm{~ms}$ D). Comparing the observed data with theoretically expected data in a Poisson distribution revealed that the shortest interspike intervals were non-Poissondistributed (A) whereas all longer $\tau$ S were Poisson-distributed.

Consequently, some interspike interval lengths - or even short sequences thereof - should be more frequent than others. For this purpose, the subsequence of $2 \tau$, i.e. $\tau_{n}$ and subsequent $\tau_{n+1}$ were plotted in a 2D-diagram (Figure 30). The color-coded figure shows exemplary for 2 different ORNs the subsequence of 3 spikes. Both ORNs revealed that for short $\tau \mathrm{s}$, especially $\tau \leq 50 \mathrm{~ms}$, the variance of data points became much smaller compared to longer $\tau$ s. A short $\tau_{n}$ was therefore unlikely followed by a long $\tau_{n+1}$, and vice versa. Furthermore, the data points of short $\tau$ s were not scattered homogeneously in the 2D map, ORN1 and ORN2 showed local maxima in the range of $\tau \approx 20-35 \mathrm{~ms}$. Some data points in these local maxima indicated sequences of stereotypic interspike intervals that were regenerated by the cell up to 4 times (ORN1). Testing a number of other ORNs showed similar results; therefore the inhomogeneous distribution of $\tau \mathrm{s}$ in the short interspike interval range in both ORNs was considered as representative for all other ORNs as well. 


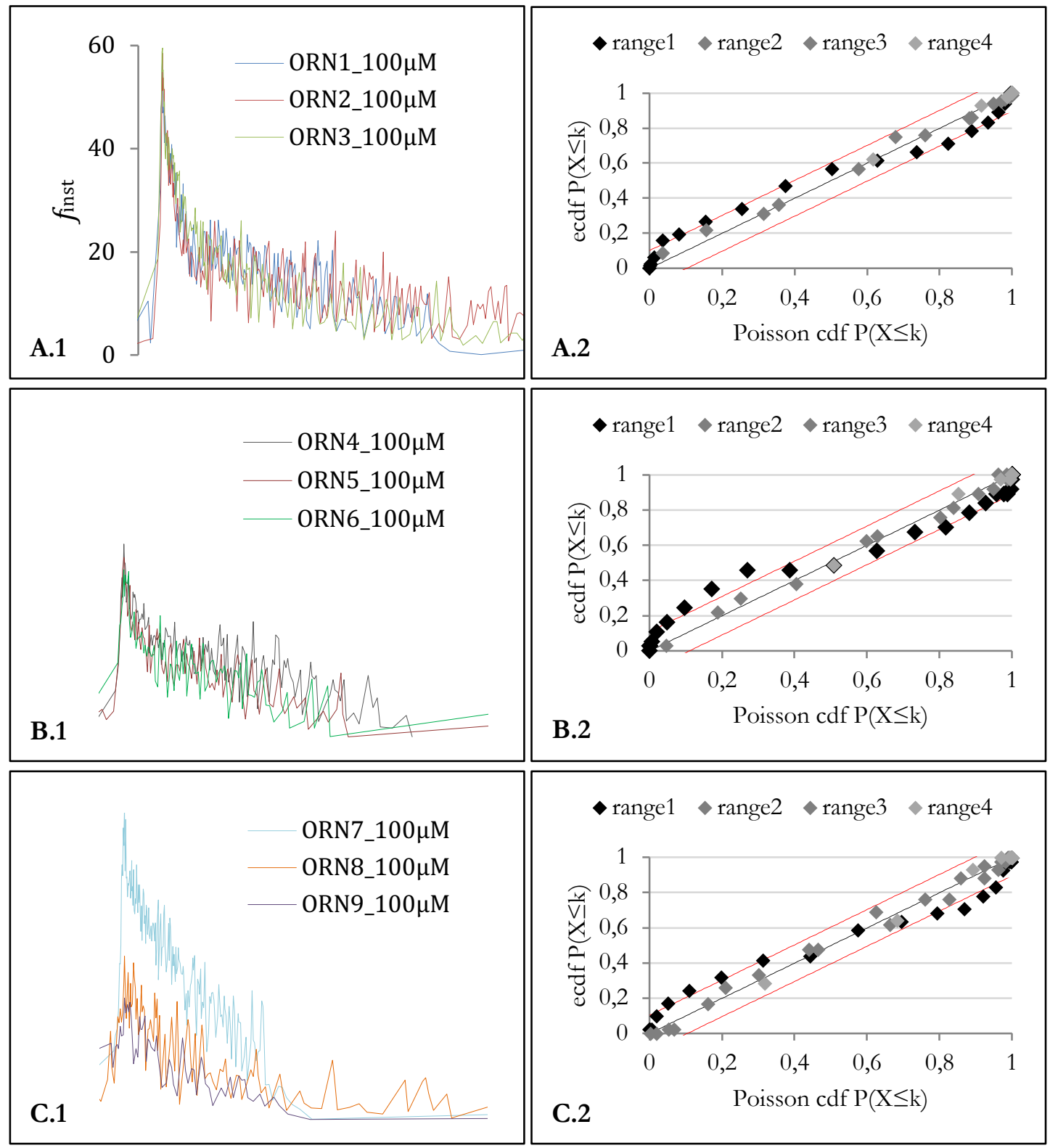

Figure 29 The distribution of interspike intervals in 9 different ORNs

Subfigure A.1, B.1 and C.1 represent the time course of instantaneous frequencies f in ORNs 1-3, ORNs 4-6 and ORNs 7-9, respectively (16 s in total, see Figure 26). The $\tau$ s of ORNs 1-3 (subfigure A.2), ORNs 4-6 (subfigure B.2) and ORNs 7-9 (subfigure C.2) are categorized into 4 equally sized interval ranges (each range sizing $83 \mathrm{~ms}, 78 \mathrm{~ms}$ and $80 \mathrm{~ms}$ for ORNs 1-3, ORNs 4-6 and ORNs 7-9, respectively). In each range the mean event rate $\lambda$ ( $=$ total number of $\tau$ s per range size) is calculated and used to estimate the resulting Poisson distribution. After fitting the Poisson distribution to the observed data such that the maximum difference of theoretical and empirical cumulative distribution functions $\mathrm{P}(\mathrm{X} \leq \mathrm{k})$ is minimized, both cumulative distribution functions are plotted: the Poisson cdf is plotted on the x-axis, the empirical cumulative distribution function is represented on the $y$-axis. In case of total conformity, the corresponding pair of values should align on the angle bisector (black linear curve). However, in all tested ORNs range1 containing the shortest $\tau$ s, i.e. $\tau \leq 100 \mathrm{~ms}$ represented by black rhombs, is (highly) significantly deviant from the expected Poisson distribution (crossing the red linear curves representing the upper and lower 1\%significance level in the Kolmogorov-Smirnov-test for discrete Poisson distribution) whereas all longer $\tau$ s are distributed in a Poissonian manner (grey rhombs). 

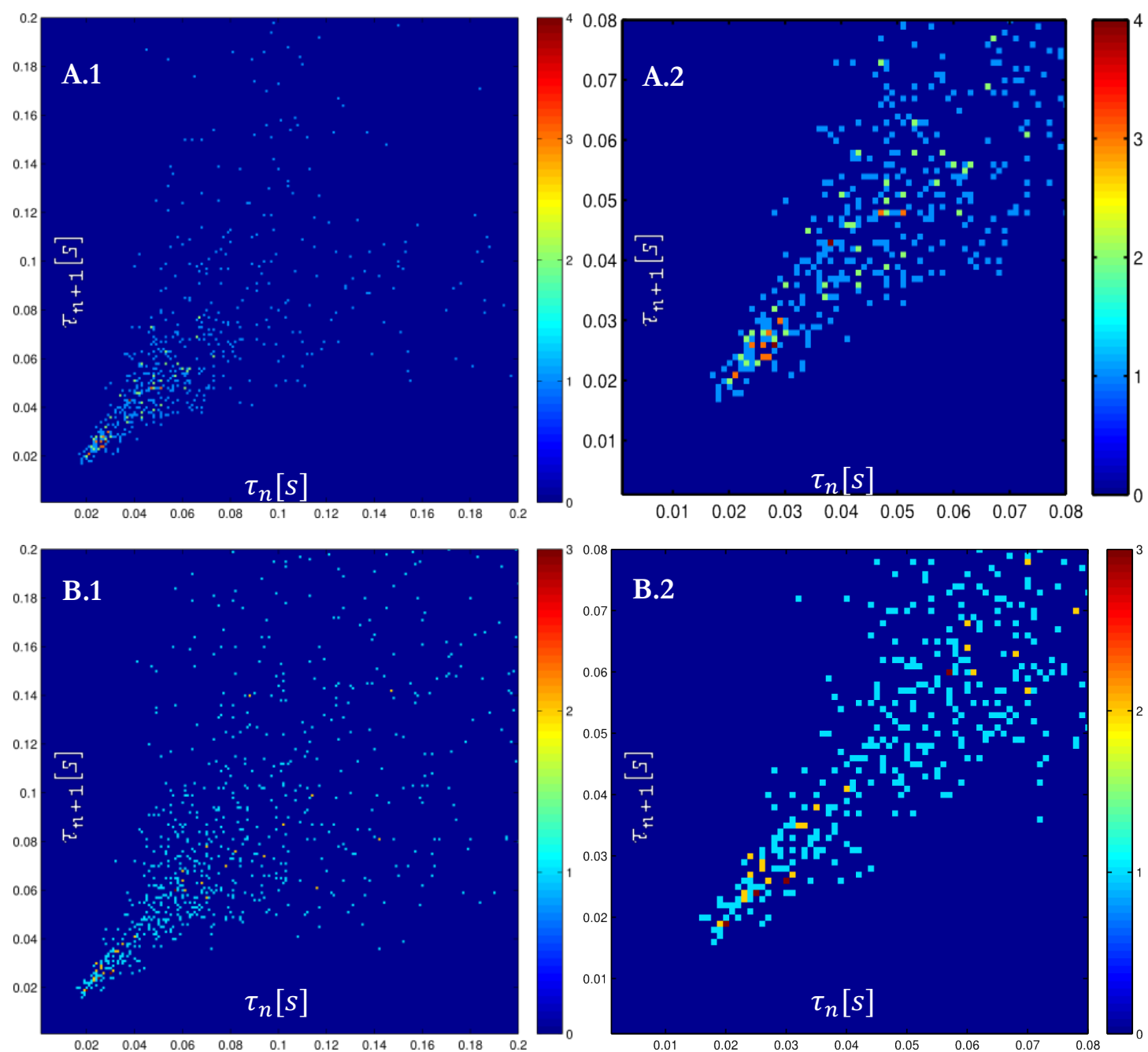

Figure 30 Color-coded 2D map of interspike interval subsequences

The $\mathrm{x}$-axis displays $\tau_{\mathrm{n}}$, the y-axis the subsequent interval $\tau_{\mathrm{n}+1}$ (bin value of $1 \mathrm{~ms}$ ). Subfigure A.1 and B.1 represent 2 different ORNs; the subfigures A.2 and B.2 are the corresponding enlargements. Both ORNs showed that for short $\tau$, especially $\tau \leq 50 \mathrm{~ms}$, the variance of data points became much smaller compared to longer $\tau$. A short $\tau_{n}$ was therefore unlikely followed by a long $\tau_{\mathrm{n}+1}$, and vice versa. Furthermore, the data points of short $\tau$ s were not scattered homogeneously in the 2D map, ORN1 and ORN2 showed local maxima in the range of $\tau \approx$ 20-35 ms. Some data points in the local maxima indicate sequences of stereotypic interspike intervals that are regenerated by the cell up to 4 times (ORN1). 


\section{Discussion}

The scope of this thesis was to understand the basic principles underlying olfactory coding in ORNs of larval Xenopus laevis. In the past 3 decades a vast amount of studies focused on the olfactory system of different species using various methods at different levels of the neuronal network.

The experimental design of this project allowed the study of the olfactory receptor neuron in both states, stationary and non-stationary. My major interest was to understand the basic properties of ORNs in order to figure out their contribution to olfactory coding. In particular, no study so far has looked into the spontaneous activity of ORNs and analyzed detailed dose-response curves of ORN ensembles in larval Xenopus laevis.

In this section the main results will be discussed in detail. But before starting, it is necessary to examine some of the methodical limitations relating to our experimental paradigms.

\subsection{Methodological limitations}

\subsubsection{The experimental design}

The experiments were designed to analyze the spontaneous firing rates and generate doseresponse curves of ORNs. Here, acute slices of nose-brain preparations were used and stained with the Ca-dye Fluo-8-AM in order to detect amino acid-sensitive ORNs. This approach allowed the detection of ORN excitatory responses to amino acids. Inhibitory responses to stimulus applications were not included in the analysis. Though several studies reported inhibitory responses to odors in aquatic animals like catfish (Kang and Caprio 1995), the existence of inhibitory responses in larval Xenopus laevis remains controversial; one study did report inhibitory responses (Vogler and Schild 1999) while another study could not confirm it (Manzini et al. 2002b).

\subsubsection{The tissue preparation}

The selected animals were chosen randomly, provided that they were at the developmental stages 51 - 54 according to Nieuwkoop and Faber (1994). This implies presumably different nutritional states of the animals, and therefore, a different homeostasis in each animal. It cannot be excluded that different hormones like insulin and leptin (Savigner et al. 
2009) or endocannabinoids (Breunig et al. 2010) had an impact on the spontaneous firing rates or the sensitivity of ORNs. However, here we used acute slices, i.e. the tissue block was dissevered from the central nervous and endocrine system, respectively. If there were any effects on the ORNs in the tissue block then those effects should have been reduced to a minimum because the tissue was incubated for at least $30 \mathrm{~min}$ at room temperature and perfused with hormone free Ringer's solution for at least 10 further minutes (see section methods and materials).

Beside endocannabinoids, purinergic signaling has been reported to affect ORNs sensitivity and activity in larval Xenopus laevis (Hegg et al. 2003). The purinergic signaling is presumed to be active in case of tissue damages (Hassenklöver et al. 2009). As acute slicing damages many neurons, it is likely that this signaling had an effect of the measured spontaneous firing rates and the sensitivity of neurons. At least the patched neurons seemed not be damaged as minimum recording time was 5 minutes, in addition to the 10 minutes of super-perfusion with Ringer's solution prior to stimulus application (+ 30 mins of incubation time). In case of any damaged neurons I would expect the neuronal activity to be changed or abolished over time due to apoptosis or necrosis. Thus, I assume that all patched ORNs remained undamaged.

\subsubsection{The preparation solutions}

Acute nose-brain slices were used to maintain the experimental conditions as close as possible to the physiological state. Such slices can prevent many artificial external effects compared to isolated cell preparations where the pericellular tissue is removed entirely and axons are cut, thereby severely affecting neuronal activities. However, in acute slice preparations, it is indispensable to remove the protective skin and the meninges which are the brain's natural barriers against the environment. In order to prevent death of the exposed neurons due to physical and chemical properties of the environmental fluids it is necessary to replace the environment with a solution simulating extracellular fluids with all its components, especially the $\mathrm{pH}$, osmolarity and salinity. Nonetheless, in nature the cilia of olfactory receptor neurons are swimming in essentially different fluids than in Ringer's solution. The highly salient extracellular solution cannot be compared to the water in ponds where the animals live naturally. Especially the high chloride concentration in the Ringer's solution might have a high impact on basal activity and responses of ORNs as the Ca-dependent chloride channel is highly dependent on chloride gradients from intracellular to environment, and not to extracellular space. 
Moreover, in acute slices the mucus layer covering the olfactory epithelium is lacking because of the perfusion with Ringer's solution. However, in the pre-metamorphic stages used in all experiments there are no glands yet producing a mucus (Hansen et al. 1998).

Taken together the experimental design, tissue preparation and the used Ringer's solution are likely to have an impact on the basal activities and response patterns of ORNs. Nevertheless, the acute slice preparations are the most physiological approaches to understand the olfactory system as a network as it mainly preserves the cellular environment of the neurons.

\subsection{Spontaneous activity and sensitivity in ORNs}

\subsubsection{Spontaneous activity in ORNs}

The spontaneous firing rates of ORNs in larval Xenopus laevis were varying from 0.24 to 5.5 per second on average. Thereby the SFRs were constant for each neuron during the whole recording time (see Figure 4). This range of firing rates is consistent with the results in different animals reported by other authors, that is, mainly below 1 up to 4 APs per second (van Drongelen et al. 1978; Getchell 1986; Rospars et al. 1994; Duchamp-Viret et al. 2000; Connelly et al. 2013; Rospars et al. 2013). In a previous study of our institute Manzini and colleagues compared SFRs of ORNs in 2 methodological preparations (Manzini et al. 2002b). Thereby, they found that the averaged SFRs of larval Xenopus laevis ORNs was $3 \mathrm{~s}^{-1}$ for isolated cells and $2-13 \mathrm{~s}^{-1}$ in acute slice preparations which would be a wider and higher range than observed here and reported by other authors. Possible explanations for this discrepancy might be the fact that the colleagues used early stage tadpoles (Nieuwkoop and Faber 48 - 54 compared to 51 - 54 here) and averaged the SFRs for all tested ORNs, including ORNs which were not responsive to amino acids. In fact, amino acid-sensitive ORNs made up only $15 \%$ of the tested population. Moreover, in this present study, only those ORNs which responded in an excitatory way were selected. ORNs which exhibit inhibitory responses might have higher SFRs compared to excitatory responsive ORNs for a clearer signal-to-noise-ratio purpose (Kang and Caprio 1995; Vogler and Schild 1999). Furthermore, Manzini and colleagues reported that they had not found any correlation between spontaneous firing rates and sensitivity of ORNs, which is another contradictory aspect of his study compared to the present results. The present results suggest that ORNs with a low firing rate are more likely to respond to concentrations below the EC-50 value 
of $8.8 \mu \mathrm{M}$ than ORNs with high activity in steady state. As the ranges of SFRs in both studies are different, a comparison of SFR and sensitivity might therefore not be applicable between both works.

To my knowledge, so far there has been no detailed on-cell voltage clamp study on spontaneous firing rates that quantitatively analyzes the mean and the variance in SFRs and shows that spontaneous activity in ORNs is a Poisson process. Extracellular recordings using micro-electrodes reported contradictory results on the Poisson character of SFRs in ORNs (van Drongelen et al. 1978; Getchell 1986; Rospars et al. 1994). However, extracellular electrodes are rather inappropriate to analyze the stochastical character of SFRs as they damage the tissue and do not necessarily represent single cell recordings. Therefore, such methods are probably inaccurate to make any conclusive statement on SFRs of single cells. Here, in on-cell recordings the Poisson character of SFRs in 35/36 ORNs could be demonstrated. In some ORNs a high variance in the SFRs could be explained by highly frequent sequences of mostly $2-4$ APs overlaying the baseline activity. These spontaneous AP bursts were mainly couplets or triplets of APs with interspike intervals of $\tau \leq 350 \mathrm{~ms}$. Such spontaneous bursts with average interspike interval of approximately $240 \mathrm{~ms}$ were reported in ORNs of adult frogs before (Rospars et al. 1994). Interestingly, the author characterized the bursts in ORNs as Poisson-distributed, while here, the interspike intervals of only $2 / 4$ ORNs were Poisson-distributed. However, by eliminating the bursts from the spiking train spontaneous activity of all neurons could be well modeled with the Poisson distribution.

So far the origin of spontaneous activity in ORNs is yet unknown despite many studies dedicated to this subject. The olfactory receptor and all following molecules in the transduction cascade like ACIII (Wong et al. 2000), cAMP levels (Nakashima et al. 2013a), CNGCs (Brunet et al. 1996) or CaCCs (Reisert 2010; Pietra et al. 2016) were discussed as possible sources. Many studies have shown that the olfactory receptor is indispensable for the presence of spontaneous activity (Serizawa et al. 2006; Reisert 2010; Connelly et al. 2013), and more than that, olfactory receptors (and the corresponding SFRs) were reported to be functionally important for anterior-posterior targeting of axon terminals to "their" glomeruli in developing vertebrates (Yu et al. 2004; Serizawa et al. 2006; Nakashima et al. 2013a). Furthermore, a recent study has discovered a specific subtype of voltage-gated sodium channels - namely cardiac type $\mathrm{Na}_{\mathrm{v} 1.5}$ - being expressed at the dendritic knob and contributing to the generator potential in ORNs (Frenz et al. 2014). The author estimated 2000 to $3000 \mathrm{Na}_{\mathrm{v} 1.5}$ channels per ORN and suggested that window currents were likely to be contributing to spontaneous activity of ORNs. Nonetheless, it is still unknown which of 
the channels and intracellular molecules mentioned above are contributing mostly to the spontaneous activity. So far important roles of AC III (Wong et al. 2000) and CNG (Brunet et al. 1996) in the generation of spontaneous APs can be ruled out. Apart from those mechanisms, there are further ion channels expressed in the ciliary ends of each ORN, for instance $\mathrm{K}^{+}$and $\mathrm{Ca}^{2+}$ ion channels (Schild and Restrepo 1998). Presumably, spontaneous activation of the G-protein-coupled receptor leads to a basal cAMP level which in turn stabilizes the resting membrane potential via HCN channels and negative feedback regulation (Nakashima $\mathrm{N}$ et al. 2013). Additionally, the stochastic character of ion channels contributes to a generator potential that occasionally leads to crossing the membrane threshold for AP generation. Taken together, all sources contribute to fluctuations of the membrane potential around the resting membrane potential and occasional crossing of the threshold for AP generation. Mathematically, the sources can be approximated as independent random variables with finite and positive variance of their activity which, when summed up, result in Gaussian noise of the membrane potential. That is the essential statement of the central limit theorem (Papoulis and Pillai 2002). The Gaussian noise results in an event rate that is Poisson-distributed. However, such assumptions can only be approximations of the reality as all sources are not actually independent. For instance, the opening probability for voltage-gated channels changes with changing membrane potential, which in turn, depends on intracellular cAMP level that influences the activity of HCN channels (Nakashima et al. N 2013; Frenz et al. 2014). Nonetheless, in this study, the Poisson character of AP rates in steady state was demonstrated for nearly all ORNs. Furthermore, the input of multiple ORNs to a virtual glomerulus was simulated. Thereby, the inputs led to a new simple Poisson distribution. However, the neurons were selected such that their SFRs were not too different, otherwise their summation would not result in a Poisson distribution (data not shown). Because of the small number of ORNs compared to 2000 - 3000 ORNs per glomerulus assumed in higher vertebrates (Ressler et al. 1994b), however, it is not possible to give reliable statements on the convergence of the obtained data. A conservative estimation of the number of ORNs converging to a glomerulus in larval Xenopus laevis can be calculated by analyzing the geometric properties of axon terminals and glomeruli at the OB level. Assuming an axonal diameter of $1 \mu \mathrm{m}$ and a glomerulus diameter of $20 \mu \mathrm{m}$ (Nezlin and Schild 2000), that 1 glomerulus is entered by 2 sub-branches of each axon which are crossing the whole glomerulus at least once (Nezlin and Schild 2005), and that half of glomerulus volume is occupied by axons and the other half by dendrites of mitral/tufted cells and periglomerular cells, the upper estimation of number of axons per glomerulus of 
amounts to $N=\frac{\frac{1}{2} * V_{\text {glom }}}{2 * V_{\text {branch }}}=\frac{\frac{1}{2} *-\frac{4}{3} * \pi * 10^{3} \mu m^{3}}{2 * \pi *\left(\frac{1}{2}\right)^{2} \mu m^{2} * 20 \mu m} \approx 67$. This theoretical number is remarkably low compared to suggested numbers in higher vertebrates. Even in the case of a $40 \mu \mathrm{m}$-wide glomerulus, the upper estimated number would be far below 1000, that is, 267. Nonetheless, the convergence of such high numbers of axon terminals will probably result in a robust Poisson-distributed spontaneous activity at presynaptic glomerular side. How exactly the SFRs are transmitted to second-order neurons and how much they contribute to spontaneous activity in mitral/tufted cells is still elusive. It is thinkable that presynaptic APs perturb the resting membrane potential by excitatory (or possibly inhibitory) post-synaptic potentials leading to membrane potential fluctuations at dendritic endings of mitral/tufted cells. By analogy with the receptor neurons in steady state such fluctuations of the membrane potential would lead to occasional generation of APs in mitral cells. It is important to keep in mind that in addition to the input by receptor neurons, there are other modulatory effects on the membrane potential in the dendritic endings of mitral cells. Paired whole-cell recordings have shown inhibitory and excitatory effects of periglomerular cells as well as dendrodendritic excitation/inhibition following stimulation (Urban and Sakmann 2002). Thus, the spontaneous firing rates in mitral/tufted cells are likely more complex than a simple linear addition of presynaptic APs. Nonetheless, that does not exclude the presence of Poisson-distributed basal activity presynaptically that is transmitted postsynaptically in steady state activity.

\subsubsection{Sensitivity}

In the experimental setup I tested single amino acid solutions within a 5-log wide concentration range from $10 \mathrm{nM}$ to $100 \mu \mathrm{M}$ and found that all ORNs responded to concentrations equal to or higher than $50 \mu \mathrm{M}$, irrespective of odorant identity. Therefore, the application of single amino acid solutions at $50 \mu \mathrm{M}$ will be sufficient to elicit a response in an ORN if the tested neuron was sensitive for that amino acid. At the other end of the concentration scale, only 3 out of 47 neurons were responsive to nanomolar-concentrated amino acid stimuli which does not exclude responses to lower concentrations than to 10 $\mathrm{nM}$. However, in this experimental setup only concentrations as low as $10 \mathrm{nM}$ were tested. Furthermore, the quantitative analysis showed that $71.7 \%$ of the neurons responded to 10 $\mu \mathrm{M}$ or higher concentrated stimuli whereas $28.3 \%$ were responsive to lower concentrations indicating an EC-50 value between $1 \mu \mathrm{M}$ and $10 \mu \mathrm{M}$. By fitting a sigmoidal dose-response curve to the observed data, the virtual EC-50 value was calculated at $8.8 \mu \mathrm{M}$. These values are in line with previous studies reporting an EC-50 value of $3-90 \mu \mathrm{M}$ in 
tiger salamander (Firestein et al. 1993), $4.4-104 \mu \mathrm{M}$ in mice (Grosmaitre et al. 2006) and 1 $-10 \mu \mathrm{M}$ in rats (Duchamp-Viret et al. 1999). A fraction of the tested ORNs responded to $10 \mathrm{nM}$ in those studies (Duchamp-Viret et al. 1999; Grosmaitre et al. 2006) as show the present results for larval Xenopus laevis. The dynamic range of stimulus detection is covering 5-log units and indicates an astonishingly diverse tuning of ORN sensitivity. A possible explanation for the wide range might be different hunger states of the chosen animals causing different influences of neuromodulatory molecules like endocannabinoids (Breunig et al. 2010), purines (Hegg et al. 2003; Czesnik et al. 2006; Hassenklöver et al. 2009), leptin and insulin (Savigner et al. 2009). Furthermore the tadpoles were at developmental stages 51 - 54 (Nieuwkoop and Faber 1994). Possibly, tadpoles in higher developmental stages have more sensitive ORNs than those at earlier stages as a previous study has already shown a "narrowing selectivity" of ORNs" response profiles in late developmental stages of larval Xenopus laevis (Manzini and Schild 2004a). Immature neurons that are still targeting "their" glomerulus may be less sensitive to odor stimuli than mature ORNs with their axons at target. A recent study has shown that $\mathrm{G}_{\mathrm{s}}$-proteins coupled to olfactory receptors are expressed simultaneously with $\mathrm{G}_{\text {olf }}$-proteins in the ORNs of mice embryos (Nakashima A et al. 2013). According to the authors, constitutively active $G_{s}$-coupled proteins would lead to an increase in the intracellular cAMP level which in turn activates protein kinase A (PKA). PKA regulates axonal targeting proteins like Neuropilin-1 or Plexin-A1 at early embryonic stages whereas glomerular segregation molecules like Kirrel2 or Kirrel3 leading to a refinement of olfactory map at bulb level - were regulated by $\mathrm{G}_{\text {olf }}$-protein coupled receptors at later embryonic stages. This way, the authors explained the regulation of molecules for glomerular targeting in the anterior-posterior axis and molecules responsible for glomerular segregation which are both driven by intracellular cAMP activity. Because $\mathrm{G}_{\mathrm{s}}$-proteins are more efficient in transducing $\mathrm{G}$-protein coupled olfactory receptor activity than $G_{\text {olf }}$ early stage embryonic ORNs possess higher cAMP levels than later stage embryos do (Nakashima A et al. 2013). As larval Xenopus laevis is a developing vertebrate, it is therefore plausible that $\mathrm{G}_{\mathrm{s}}$-proteins are gradually replaced by $\mathrm{G}_{\text {olf }}$-proteins in later developmental stages. This would lead to reduced intracellular cAMP levels and therefore lower spontaneous activity in ORNs (Reisert 2010) but higher sensitivity because in mature ORNs $G_{\text {olf }}$ has a higher turnover of guanosinetriphosphate (GTP) to guanosinediphosphate (GDP). By expressing higher amounts of phosphodiesterase (PDE) molecules it is possible to precisely detect temporal changes in intracellular cAMP and effectively govern the activation of the canonical cAMP-mediated pathway (Nakashima A et al. 2013). However, these results of embryonic developmental stages in mice cannot be 
completely applied to the amphibian larval stage of Xenopus laevis as, unlike embryonic mice, Xenopus laevis larvae are free swimming organisms in their natural environment and are already capable of olfaction.

\subsubsection{Inverse relation of SFR and sensitivity}

To my knowledge, so far, only one research group described the relation between SFRs and ORN sensitivity. The two processes were not correlated in any case (Manzini et al. 2002b). However, the present results suggest the opposite; the slowest firing ORNs are more sensitive than ORNs with higher activity in steady state. In section 4.2.1, the discrepancies between this study and Manzini's study were already discussed. Furthermore, possibly interfering factors like an unfavorable signal-to-noise ratio in fast firing ORNs or direct impact of the chosen amino acid were excluded. Even in the most unfavorable constellation, the spiking rate of an ORN activated by a $1 \mu \mathrm{M}$ stimulus application was approximately 5 times higher than its SFR. Thus, all responses to the stimulus concentration that classified an ORN as high-sensitive or low-sensitive (see section 3.1.2) were clearly distinguishable. Although alanine-sensitive ORNs are predominantly lowsensitive ORNs, the difference to arginine and methionine-sensitive ORNs is statistically not significant.

The inverse relation between sensitivity and SFRs is interesting as it has not been reported before. A possible explanation for this phenomenon was discussed in the previous section 4.2.2 (replacement of $G_{\mathrm{s}}$ by $G_{\text {olf }}$ across development). Both, SFRs and sensitivity of ORNs could be explained by changing cAMP-levels at different larval stages. Alternatively, the presence of voltage-dependent sodium channels may offer another explanation: a less negative membrane resting potential (leading to a higher basal spontaneous activity) would lead to the inactivation of a bigger fraction of voltage-gated sodium channels which in turn would reduce the cell sensitivity (Firestein and Werblin 1987).

Moreover, the influence of endocannabinoids, purines and other neuromodulators on spontaneous activity and sensitivity of ORNs was reported previously (Hegg et al. 2003; Hassenklöver et al. 2009; Breunig et al. 2010). Whether the neuromodulators have an impact on the inverse relation of SFR and sensitivity is nonetheless unknown. 


\subsection{ORNs and olfactory coding}

\subsubsection{Peripheral olfactory coding at single cell level}

Since many studies were dedicated exploring olfactory coding at olfactory bulb and higher levels (Ressler et al. 1994b; Lledo et al. 2005; Poo and Isaacson 2009) it was desirable to understand the basic principles underlying stimulus-induced changes in the primary sensory neurons at the first level of interaction between olfactory stimulus and the sensory system. Previously, many studies recounted dose-response curves for ORNs in different species, among others in the rat and the frog (Duchamp-Viret et al. 1999; Duchamp-Viret et al. 2000), the mouse (Ma et al. 1999; Grosmaitre et al. 2006) and the tiger salamander (Firestein et al. 1993). They all suggested a monotonously increasing number of APs and frequency with increasing stimulus concentration.

In the current thesis, I investigated the maximum instantaneous post-stimulation frequency (defined as the reciprocal value of the minimum interspike interval $\tau_{\min }$ ) and the number of APs in the ORNs of larval Xenopus laevis. It was of interest to calculate doseresponse curves and to decide which parameter, frequency or number of APs, was better suited to encode information on odor concentration at the single cell level. Latency coding at the ORN level has not been analyzed within the scope of this project. The median average of instantaneous frequencies ranged thereby from $9.39 \mathrm{~Hz}$ to $26.00 \mathrm{~Hz}$. A saturation of the frequency increase was seen at $50 \mu \mathrm{M}$, which was identical to the saturation concentration described for the number of responsive receptor neurons. Interestingly, the receptor neurons responded with widely different frequencies to the same stimulus concentration; the frequencies triggered by $100 \mu \mathrm{M}$-concentrated stimuli ranged from $5.64 \mathrm{~Hz}$ to $59.17 \mathrm{~Hz}$ corresponding to minimum interspike intervals of $\tau_{\min } \approx$ $178 \mathrm{~ms}$ to $\tau_{\min } \approx 17 \mathrm{~ms}$, respectively. Interestingly, the same maximum frequency of approximately $60 \mathrm{~Hz}$ was also found in adult frog Rana ridubunda (Duchamp-Viret et al. 2000). Moreover, when comparing stimulus applications of a difference of 2-log units occasionally elicited instantaneous frequencies of similar magnitude (minimum frequency to $100 \mu \mathrm{M}$ was in the same range as to $1 \mu \mathrm{M})$. Therefore, instantaneous maximum frequencies were mainly concentration invariant. The frequency ranges in response to a 100 $\mu \mathrm{M}$-concentrated stimulus reflects the minimum interspike intervals that can be generated by ORNs in larval Xenopus laevis. Notably, these minimum interspike intervals are in the same range as the membrane time constants reported for different species, ranging from $\tau_{\mathrm{m}} \approx 40 \mathrm{~ms}$ to $100 \mathrm{~ms}$ (Schild and Restrepo 1998). As the membrane time constant is a product of cellular membrane capacitance (and therefore of the cellular membrane surface 
area) and the resting membrane resistance (cellular size and shape) $\tau_{m}=C_{m} * R_{m}$ the passive physical properties contribute partly to the magnitude of baseline activity and stimulus induced frequencies in neurons. Mammals, for instance, are reported to have smaller ORNs than amphibians, resulting in a smaller cell capacitance in mammals (Lynch and Barry 1989). Consequently, one study discovered that both the spontaneous and stimulus-induced firing rates of ORNs in rats were higher than in frogs (Duchamp-Viret et al. 2000). From the aspect of energy ecology this appears to be reasonable because bigger ORNs (with corresponding higher cellular capacitance) in poikilothermic amphibians result in lower basal and stimulus-induced activity, and therefore, in a lower total energy consumption compared to homeothermic mammals (however, at the expense of speed). Variation of SFRs and stimulus-induced frequencies within the same species may therefore simply reflect different geometric properties of the patched cells. However, the passive physical properties of ORNs contribute only partly to the measured frequencies as the biological complexity of the cell cannot be mechanistically reduced to that of a simple RCelement. The expression of ion channels and the cytosolic machinery of second messengers as well as response termination molecules have a crucial influence on spontaneous and stimulus-induced cell activity. For instance, high voltage-activated $\mathrm{Ca}^{2+}$-channels were found to be clustered and colocalized with large conductance potassium BK-channels at ORN soma (Bao et al. 2015). The calcium influx triggered by the AP activates thereby BKchannels which leads to an accelerated repolarization and therefore enables the ORN to elicit high frequent AP rates. The role of intracellular cAMP-levels and voltage-gated sodium channel $\mathrm{Na}_{\mathrm{v} 1.5}$ for SFRs has been discussed above (see section 4.2.1). Thus, differences in spontaneous and stimulus induced frequencies are likely to reflect the physical and biological properties of the cell rather to encode information in a sense that a certain frequency codes unambiguously a defined concentration or odor epitope. The only concentration dependent behavior of the ORN was such that - except of a few cells - an individual cell responded mainly in a manner that $f_{\max }\left(c_{2}\right)>f_{\max }\left(c_{1}\right)$, if $c_{2}>c_{1}$. Testing the number of APs as possible coding parameter yielded in a similar result as obtained for the maximum instantaneous frequencies. The ORNs generated 12 APs in the event of $10 \mathrm{nM}$-concentrated stimulus up to maximum 200 APs in case of the highest applied concentration. On average, ORNs generated a range of number of APs from approximately 13 to 135 . Thereby, similarly to the maximum instantaneous frequencies, the numbers of APs to different stimulus concentrations were widely overlapping and therefore nearly concentration invariant. In order to test the hypothesis that the maximum instantaneous frequency and number of APs represent the same information content 
regarding stimulus concentration coding both parameters were correlated which yielded a high correlation coefficient.

Taken together, the dose-response curves at the single cell level revealed that information coding on olfactory stimuli was unlikely to be represented in absolute AP rates or number of APs in single receptor neurons. Both parameters were highly correlated with each other and presumably represent the same information content. And, as both parameters were mainly concentration invariant in the interneuronal comparison, information coding at single cell level alone would likely be insufficient. However, in the natural environment it would be crucial for the animal's survival to distinguish between 2 concentrations of a 2 $\log$ unit difference in order to navigate to the odor source.

\subsubsection{Peripheral olfactory coding in neuronal ensembles and stationarity}

Testing olfactory coding in ORN ensembles by summation of the original spike trains of 9 ORNs resulted in an increase of number of activated neurons, frequency, number of APs and response duration with increasing stimulus concentration. Thereby, frequency and number of APs are concentration-dependent and ranged from approximately 15 APs (10 $\mathrm{nM})$ to more than 90 APs $(100 \mu \mathrm{M})$ for a bin width of $500 \mathrm{~ms}$. If we assume simplistically that the addition of presynaptic APs will lead to addition of postsynaptic EPSPs in mitral dendrites which themselves will initiate an AP by crossing the membrane threshold, then a higher rate of presynaptic APs will lead to an earlier generation of APs postsynaptically. Therefore, the highly concentration-dependent AP rates in neuronal ensembles might be a possible mechanism underlying temporal coding of stimulus concentrations. Nonetheless this assumption is a great simplification as it is known that latencies are already present presynaptically (Spors et al. 2006) and the large influence of periglomerular and interdendritical inhibition as well as excitations by AP propagation have been reported (Urban and Sakmann 2002).

The cumulative responses offer an approach to the initial question of what is characteristic for steady state as vague responses at single cell level resulted in clearcut responses when the traces of 6 cells only were added up. The previous results of this thesis already revealed that SFRs of ORNs have a Poisson character and that the convergence of multiple SFR sources results in another Poisson distribution. Assuming that the convergence of (similarly tuned) ORNs to a glomerulus would yield a Poisson distribution of AP rate then this state could be defined as stationarity (event rate $\lambda$ is constant). Every significant divergence, i.e. a too high or too low AP rate (in a given time range), can be interpreted as non-stationarity. To show this practically, the SFR of 6 ORNs were averaged and a Poisson distribution was 
assumed (3.3.2). The cumulative responses showed that the observed data points were significantly different than predicted in the hypothetical Poisson distribution for high concentrations. At threshold concentrations, however, the bin width of $500 \mathrm{~ms}$ was too big and therefore generated too few data points in order to be statistically significant. Therefore, I compared the first $10 \mathrm{~s}$ after stimulus application with the following $20 \mathrm{~s}$. In case of stationarity no difference should have been seen. However, even in the case of a 10-nM concentrated stimulus application, the responses were clearly and highly significantly distinguishable from the steady state. According to these data, the olfactory system could perfectly extract stimulus-induced signals from intrinsic noise of the system (irrespective of the origin and the function of spontaneous activity). The convergence of multiple receptor neurons to a glomerulus will enable the system to filter the signal from noise by setting a "cut-off” value that determines non-stationarity. Physiologically, this can be achieved by varying the dendritic membrane potential relative to the threshold for AP generation, and changing physical and biological properties of the dendritic plasma membrane. The addition of EPSPs upon excitation or of IPSPs upon inhibition (possibly via inhibitory interneurons) will lead to the activation of a tertiary (cortical) neuron which indicates the presence of a stimulus. Such a coding system would provide 3 major advantages: 1) the intrinsic noise of the system can be eliminated and signals at threshold concentrations are clearly distinguishable, 2) the sensitivity of the whole system is much higher than the sensitivity of its single components. For instance, if theoretically 100 ORNs converged to a glomerulus and each ORN had a SFR of approximately 1 per second, then a fraction of only $31 \%$ of the neurons would need to fire one single additional AP (assuming a 1s-bin) in order to be a highly significant signal $(\mathrm{p}<0.001)$ that could lead to the activation of a tertiary neuron. In the case of a convergence of 1000 ORNs then a fraction of only $9.9 \%$ of the converging ORNs would need to fire one single AP more in order to be significantly different. Figure 31 illustrates the growing sensitivity along with the increasing number of ORNs converging to a glomerulus. However, with a growing glomerular diameter and the limitation of physical space, there is a trade-off between sensitivity (size of glomerulus) and specificity (total number of glomeruli), making the architecture of olfactory bulb the result of a 2-dimensional optimization problem. 3.) The "disadvantage" of intrinsic noise can be turned into the "advantage" of a wider coding range, whereby not only excitatory but also inhibitory responses are possible due to a basal activity level. 


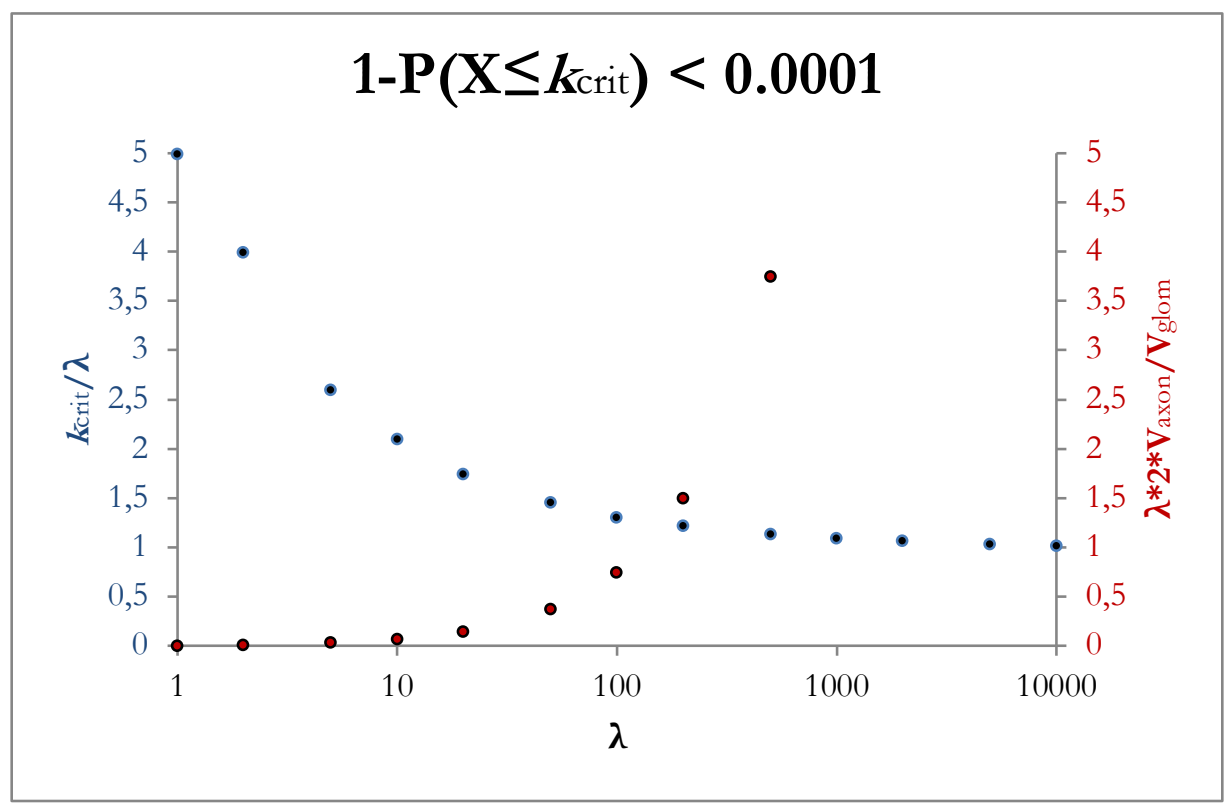

Figure 31 Sensitivity and specificity of glomeruli

This figure illustrates the growing sensitivity of the olfactory system (blue line) with an increased glomerular input (red line). The sensitivity is limited by physical space. Left vertical axis represents the ratio of critical $k_{c r i t}$ and event rate $\lambda$ which is identical with the number of input neurons when assuming each neuron has constant SFR of approximately 1 AP per second. Thereby, $\mathrm{k}_{\text {crit }}$ is the event with $\mathrm{P}\left(\mathrm{X}<\mathrm{k}_{\mathrm{crit}}\right) \geq 0.999$ in a Poisson distribution. The horizontal axis represents convergence of $\lambda *$ ORNs with SFR of 1 AP per second. With a growing number of inputs the ratio converges asymptotically to 1, i.e. the higher the number of inputs the lower the needed number of activated neurons will be in order to encode the presence of a signal. The second vertical axis (red) represents the ratio of axonal volume ( 2 branches per ONR, $1 \mu \mathrm{m}$ axonal diameter) to glomerular volume (20 $\mu \mathrm{m}$ in diameter) with growing input of $\lambda^{*}$ ORNs. At $\lambda=100 \mathrm{k}_{\text {crit }}$ equals to 131 , however, $75 \%$ of the glomerulus would be filled with axonal terminals of 100 ORNs.

\subsection{ORN response dynamics}

\subsubsection{ORN response profiles}

Analyzing the post-stimulation time course of single ORNs revealed that groups of neurons showed astonishing similarities in their response profiles. The ORNs of different animals, at different experimental days and with various stimuli responded nearly identical to the same stimulus concentration. One possible explanation for this phenomenon could be the expression of more than one receptor type per ORN. There is evidence that at least 3 signaling pathways are existing in larval Xenopus laevis for amino acid-sensitive ORNs (Sansone et al. 2014). Moreover, a previous study has revealed that receptor neurons exhibit 204 different response profiles in 283 tested amino acid sensitive ORNs. Thereby, 
$115 / 283(\approx 40 \%)$ ORNs had response profiles that re-occurred more than once. Moreover, repeated stimulation of an ORN with the same stimulus concentration resulted in similar response traces. The author, therefore, suggested that larval stages of Xenopus laevis may express more than 1 odorant receptor type per ORN (Manzini et al. 2007). It is thinkable that the combination of different receptor types will determine the response profile of an individual neuron. Neurons expressing the same combination of receptor types could possibly show similar response profiles and response kinetics.

Another explanation for this finding could be the expression level of Bk-channels. Bkchannels have been shown to be important for the speed of AP generation in ORNs (Bao et al. 2015). As Bk-channels are co-localized with high voltage-gated $\mathrm{Ca}^{2+}$-channels which in turn are clustered and of limited number, expression of a defined number of Bkchannels on the cell surface could lead to a defined response kinetic. ORNs expressing a high number of Bk-channels would be generating higher stimulus-induced AP frequencies than those with a low number of Bk-channels.

\subsubsection{Two decays, two distributions}

Alongside the different response profiles in ORNs, the stimulus-induced time course revealed a biphasic decay for 7/9 ORNs when being exposed to high-concentrated stimulus. In the case of linearity one simple exponential decay should be expected as the

stimulus solution was washed out with a constant flow, i.e. it is expected that $\frac{\mathrm{dC}}{\mathrm{dt}}=-\mathrm{kC}$. The non-linear relation of instantaneous frequency and stimulus concentration at short interspike intervals can possibly be explained by 2 different mechanisms. First, every stimulus-induced signal in sensory neurons will be terminated by two cellular adaptation pathways. For ORNs numerous pathways are important for the response termination (Kaupp 2010). Some authors distinguish between short- and long-term adaptation mechanisms. Possibly, very short interspike intervals induced by saturation concentrations and associated with very high intracellular $\mathrm{Ca}^{2+}$-concentrations are terminated by mechanisms involving molecules directly depending on the transmembrane $\mathrm{Ca}^{2+}$-gradient, such as the Na-Ca-Exchanger or the Na-Ca-K-Exchanger (Pyrski et al. 2007). This termination mechanism is then followed by slower adaptation mechanisms such as phosphorylation and capping of OR by $\beta$-arrestin, inhibition of ACIII by Calciumdependent CaM-Kinase II or hydrolysis of cAMP by PDE (Kaupp 2010). Therefore, the 2phase decay might be the result of 2 different adaptation mechanisms, the short-term and long-term adaptation, respectively. Another explanation for the non-linear relation may be 
found in the expression of Bk-channels. In order to generate short interspike intervals, the activation of Bk-channels is indispensable (Bao et al. 2015). After generating the shortest possible interspike intervals, the inactivation of Bk-channels will lead to a fast decrease of the instantaneous frequency, and therefore, to a first fast decay and a second slower decay. However, both mechanisms are not exclusive. It is likely that both contribute to the nonlinear relation of frequency and concentration at high stimulus concentrations.

In the previous sections stationarity was defined as a Poisson process with a constant event rate $\lambda$. However, this does not exclude that in stimulated state the length of interspike intervals was not Poisson distributed, just with a different event rate. And as the time course reveals 2 different decay kinetics it was interesting to know if in both cases the lengths of interspike intervals were Poisson-distributed. Analyzing the length of the interspike intervals $\tau$ revealed that all $\tau>100 \mathrm{~ms}$ were robustly Poisson-distributed. However, in case of shorter interspike intervals the distribution was significantly nonPoisson, which means that not all possible interspike interval ranges are "covered" homogenously. Some interspike interval lengths occur more often than others. To visualize this phenomenon the subsequence of 3 APs and 2 interspike intervals were plotted for each cell (Figure 30). The receptor neurons show interspike interval ranges of local maxima and minima with some identical interspike interval subsequences. This finding underlines the non-Poisson character in the length of interspike intervals for short $\tau$. The limit of $100 \mathrm{~ms}$ was set arbitrarily. Nonetheless, the results show clearly that for short interspike interval ranges the cell generates APs in a less probabilistic manner. There are 2 possible explanations for this phenomenon: Firstly, as the binding of odor molecules to olfactory receptors lasts $\sim 1 \mathrm{~ms}$ only (Bhandawat et al. 2005) and the adaptation to short interspike intervals lasted several $100 \mathrm{~ms}$, applying saturation concentrations of stimuli to the cilia of ORNs could therefore possibly lead to re-binding of ligands to the receptors due to a prolonged stimulus washout and turbulences of the solution flow. In such case, fast adaptation and re-stimulation can lead to a sequential decrease of frequency. Secondly, the activation of a low number of Bk-channels enables the cell to generate short interspike intervals (Bao et al. 2015). Successive inactivation of Bk-channels can lead to a limited number of states in the cell which can generate limited ranges of interspike intervals. Thus, the maximum firing frequency would be elicited by the activation of all Bk-channels and the subsequent inactivation would lead to abrupt decreases of AP frequency and adaptation. 


\section{Summary}

The scope of this thesis was to investigate the activity of ORNs in larval Xenopus laevis in the absence and presence of adequate stimuli. In order to understand the basic principles underlying olfactory coding at first level of stimulus detection it was important to provide a comprehensive and quantitative analysis of ORN activity.

Acute nose-brain slice preparations of the olfactory epithelium, the olfactory nerves and the anterior part of the brain including the olfactory bulb were used as an experimental approach to address these questions. The olfactory receptor neurons were incubated with the calcium dye Fluo-8-AM and following $\mathrm{Ca}^{2+}$-imaging enabled the detection of amino acid-sensitive ORNs. Patching the amino acid-sensitive ORNs in the cell-attached voltage clamp mode allowed the recording of both spontaneous and stimulus induced activities. The stimuli were delivered to the ORNs in a manner that first, the lowest concentration at $10 \mathrm{nM}$, was applied and followed by the next highest concentration after a washout period of minimum $60 \mathrm{~s}$. The stimulus' concentration range covered a 5-log unit wide range, from $10 \mathrm{nM}$ to $100 \mu \mathrm{M}$.

All ORNs $(n=46)$ showed activity in the absence of obvious stimulus. Thereby, the SFRs of ORNs were constant during recording time but differed markedly across different neurons, ranging from 0.24 to 5.5 APs per second on average. Furthermore, it was shown that in almost all ORNs the spontaneous activity was a Poisson process and the SFRs were a good first estimate of the event rate $\lambda$. In addition, not only was the rate of APs per defined bin Poisson distributed but also the lengths of interspike interval $\tau$. There have been contradictory statements on the Poissonian character of spontaneous activity in ORNs, however, all previous studies used intracellular microelectrodes to record single cell activity. To my knowledge, this is the first time that the Poisson character of spontaneous activity in ORNs has been demonstrated unambiguously using the cell-attached patch clamp mode. As to the origin of the stochastic character of spontaneous activity it remains unclear to identify the main source of intrinsic noise in receptor neurons. Possibly, molecules involved in the canonical signal transduction pathway as well as the inherent stochastic character of ion channels, for instance the voltage gated sodium channel $\mathrm{Na}_{\mathrm{v} 1.5}$, build independent random variables with finite and positive variance of their activity. In sum their activities result in Gaussian noise of the membrane potential below the threshold potential to generate APs. Occasional crossing of the threshold potential elicits spontaneous APs with event rate $\lambda$, which is Poisson-distributed. 
The application of stimuli revealed diverse tuning of ORNs, 3/46 ORN responding to concentrations in the nanomolar range, all of them responding to stimulus concentrations as high as $50 \mu \mathrm{M}$. The calculated virtual EC-50 value is $8.8 \mu \mathrm{M}$. The calculation of dosedesponse curves revealed surprisingly that both instantaneous frequencies and number of APs are mainly concentration invariant, and that they cover a range of 3.19 to $59.17 \mathrm{~Hz}$ and 13 APs to 135 APs, respectively. Thereby, the responses to stimulus concentrations of 2-log units' difference are widely overlapping. In contrast, cumulative responses of ORNs are clearly indicative of the applied stimulus concentrations and unveil cellular responses to thresholds concentrations that elicit elusive responses in single cell activity. It is therefore assumed that concentration coding at the level of the olfactory epithelium is not performed via frequencies or number of APs but that these are characteristic features of a cell representing its physical (e.g. the membrane capacitance) and biological (e.g. the expression of Bk-channels) properties. The coding of stimulus identity is rather performed in neuronal ensembles converging to glomeruli. Supposing that arrival of presynaptic APs will lead to addition of postsynaptic EPSPs (or IPSPs) could provide an explanation for the temporal dynamic of (spatial) combinatorial coding at olfactory bulb level. Modeling spontaneous activity as a Poisson process enabled the clear detection of ORNs' responses to threshold concentrations. Therefore, the Poisson model can help to distinguish stationarity from non-stationarity at the ORN level. Furthermore, the stochastic model illustrates the benefits of a converging system like that of the olfactory system: it helps eliminate intrinsic noise, leads to a wider dynamic coding range and increase of sensitivity of the whole system compared to the sensitivity of its single components.

Finally, 6/46 ORNs showed similar response patterns. Interestingly, in these ORNs the frequency decay following high stimulus application is biphasic. Moreover, all short interspike intervals $\tau \leq 100 \mathrm{~ms}$ were non-Poisson-distributed whereas longer $\tau$ s were. Possible explanations for this phenomenon may be two different response termination mechanisms, for instance, a fast adaptation via $\mathrm{Na}^{+}-\mathrm{Ca}^{2+}$-Exchanger and a second slower adaptation via the phosphorylation of the olfactory receptor or the hydrolysis of cAMP. Another plausible explanation may be the activation and deactivation of large conductance potassium Bk-channels. 


\section{$6 \quad$ Appendix}
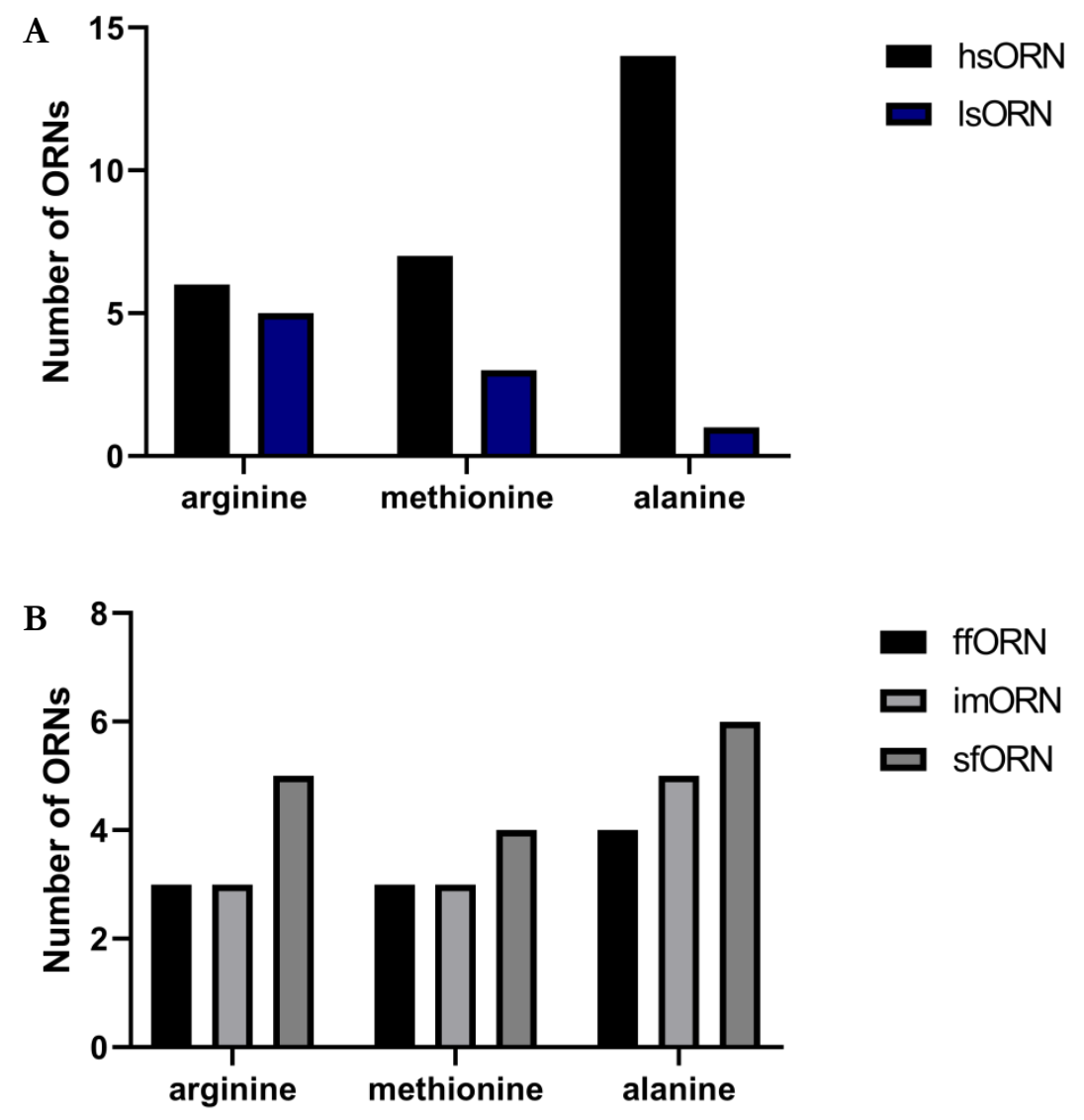

Figure 32 ORNs sensitive to arginine, methionine and alanine

A. ORN response to arginine $(\mathrm{n}=11)$ consisted of an almost equally sized number of $1 \mathrm{~s} O \mathrm{RN}$ and hsORNs, respectively. Among methionine-sensitive ORNs $(n=10) 7$ were low-sensitive and 3 were highly sensitive which was in line with the overall portion of lsORNs and hsORNs in all ORNs. Alanine-responsive ORNs $(\mathrm{n}=15)$, however, were mostly low-sensitive. The differences between the 3 groups is no significant (the $\chi^{2}$-test, $\mathrm{p}=0.07$ ) $\mathbf{B}$. Proportion of slow, intermediate and fast firing ORNs among amino acid sensitive ORNs. Methionine-sensitive ORNs were represented by each group almost equally and therefore the number of lsORNs and hsORNs in methionine-sensitive ORNs resemble most the overall distribution in all 46 ORNs. 5 out of 11 arginine-sensitive ORNs were sfORNs and could therefore explain why there was a slightly bigger portion of hsORNs among arginine-sensitive cells. Finally, the remarkable high portion of lsORNs in alanine sensitive neurons could not be explained by the SFR. Most alanine-sensitive ORNs were sfORNs and the portion of ffORNs was the lowest among all 3 amino acids. Therefore, the opposite result would have been expected assuming that sfORNs are more sensitive than ffORNs. No statistical difference among the 3 different groups regarding SFRs $\left(\chi^{2}\right.$-test, $\left.\mathrm{p}>0.05\right)$. 


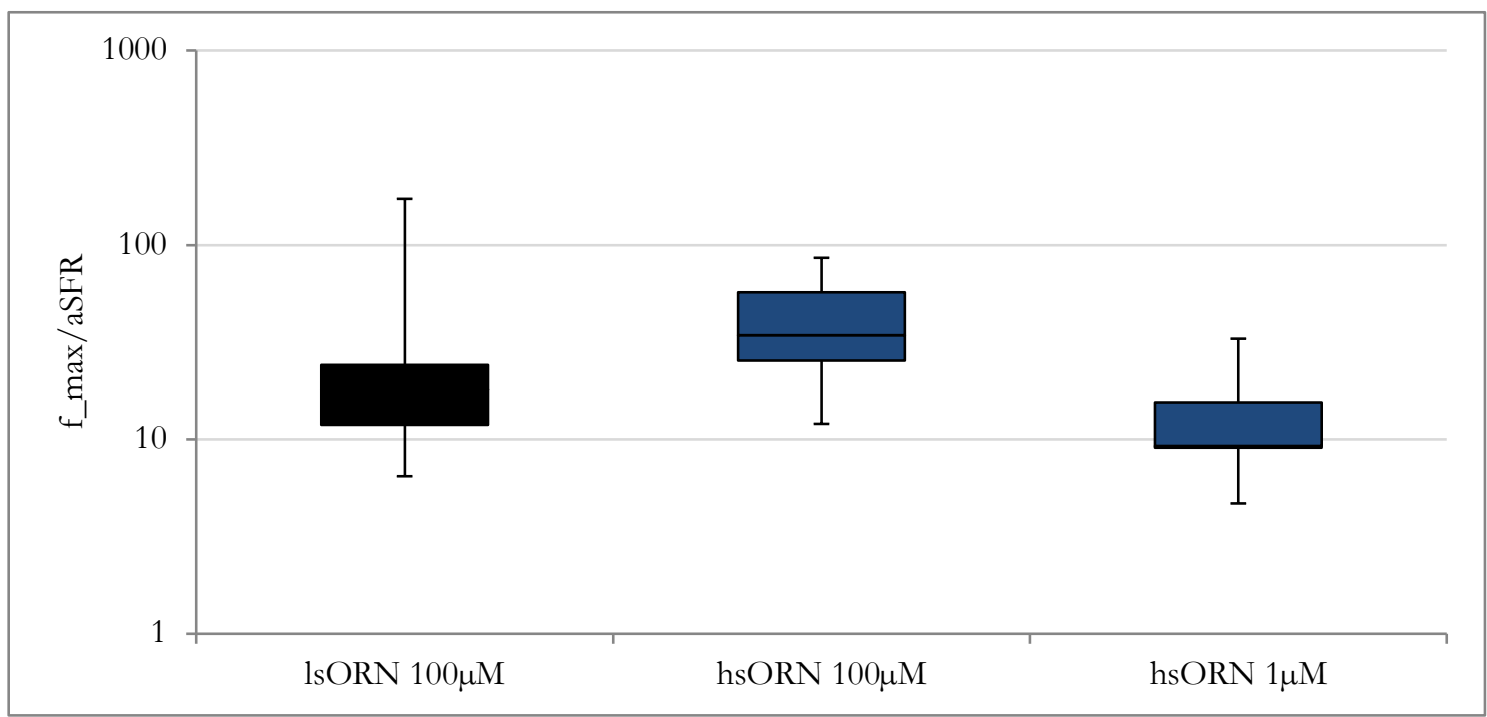

Figure 33 Ratios of $\mathbf{f}_{\max }$ and aSFR in low and highly sensitive ORNs

The ratio is determined as the maximum instantaneous firing rate $f_{\max }$ and the averaged spontaneous firing rate (aSFR) depending on the applied concentration. For $100 \mu \mathrm{M}$ concentrated solutions there was a difference for lsORNs and hsORNs. However, the lowest $f_{\text {max }} /$ aSFR was around 6.5 in lsORNs, i.e. the response was still very clear, even for the most unfavorable constellation. For the critical concentration of $1 \mu \mathrm{M}$, the lowest ratio was not far below the ratio measured for $100 \mu \mathrm{M}$ (being approximately 4.7). On the other hand, the highest ratio was measured in lsORNs, fmax being 172.5 fold aSFR. The table below the figure lists all calculated ratio values.

Table 8 Ratios of $\mathbf{f}_{\max }$ and aSFR in low and highly sensitive ORNs

\begin{tabular}{|cccc|}
\hline & lsORN 100 $\mu$ M & hsORN 100 $\mu$ M & hsORN $1 \mu \mathrm{M}$ \\
\hline Minimum & 6,46 & 12 & 4,68 \\
\hline Q1 & 11,86 & 25,44 & 9,02 \\
\hline Median & 18,04 & 34,35 & 9,24 \\
\hline Q3 & 24,17 & 57,07 & 15,46 \\
\hline Maximum & 172,53 & 85,85 & 32,95 \\
\hline
\end{tabular}




\section{Bibliography}

Atanasova B, Graux J, El Hage W, Hommet C, Camus V, Belzung C (2008): Olfaction: A potential cognitive marker of psychiatric disorders. Neurosci Biobehav Rev $\underline{32}$, 1315-1325

Avila VL, Frye PG (1977): Feeding Behavior in the African Clawed Frog (Xenopus laevis Daudin). Herpetologica 33, 152-161

Baly C, Aioun J, Badonnel K, Lacroix M-C, Durieux D, Schlegel C, Salesse R, Caillol M (2007): Leptin and its receptors are present in the rat olfactory mucosa and modulated by the nutritional status. Brain Res 1129, 130-141

Bannister LH, Dodson HC (1992): Endocytic pathways in the olfactory and vomeronasal epithelia of the mouse: Ultrastructure and uptake of tracers. Microsc Res Tech $\underline{23}$, 128-141

Bao G, de Jong D, Alevra M, Schild D (2015): Ca2+-BK channel clusters in olfactory receptor neurons and their role in odour coding. Eur J Neurosci 42, 2985-2995

Bhandawat V, Reisert J, Yau K-W (2005): Elementary Response of Olfactory Receptor Neurons to Odorants. Science $\underline{308}, 1931-1934$

Bloes DA, Kretschmer D, Peschel A (2015): Enemy attraction: bacterial agonists for leukocyte chemotaxis receptors. Nat Rev Microbiol 13, 95-104

Breipohl W, Laugwitz HJ, Bornfeld N (1974): Topological relations between the dendrites of olfactory sensory cells and sustentacular cells in different vertebrates. An ultrastructural study. J Anat 117, 89-94

Brennan PA, Kendrick KM (2006): Mammalian social odours: attraction and individual recognition. Philos Trans R Soc Lond B Biol Sci $\underline{361}, 2061-2078$

Breunig E, Manzini I, Piscitelli F, Gutermann B, Marzo VD, Schild D, Czesnik D (2010): The Endocannabinoid 2-Arachidonoyl-Glycerol Controls Odor Sensitivity in Larvae of Xenopus laevis. J Neurosci 30, 8965-8973

Brinkmann A, Schild D (2016): One Special Glomerulus in the Olfactory Bulb of Xenopus laevis Tadpoles Integrates a Broad Range of Amino Acids and Mechanical Stimuli. J Neurosci $\underline{36}$, 10978-10989

Brunet LJ, Gold GH, Ngai J (1996): General Anosmia Caused by a Targeted Disruption of the Mouse Olfactory Cyclic Nucleotide-Gated Cation Channel. Neuron 17, 681693

Buck L, Axel R (1991): A novel multigene family may encode odorant receptors: A molecular basis for odor recognition. Cell $\underline{65}, 175-187$

Buehlmann C, Graham P, Hansson BS, Knaden M (2014): Desert Ants Locate Food by Combining High Sensitivity to Food Odors with Extensive Crosswind Runs. Curr Biol 24, 960-964 
Busenbark KL, Huber SJ, Greer G, Pahwa R, Koller WC (1992): Olfactory function in essential tremor. Neurology 42, 1631-1631

Caprio J (1978): Olfaction and taste in the channel catfish: An electrophysiological study of the responses to amino acids and derivatives. J Comp Physiol 123, 357-371

Chao TI, Kasa P, Wolff JR (1997): Distribution of astroglia in glomeruli of the rat main olfactory bulb: Exclusion from the sensory subcompartment of neuropil. J Comp Neurol $\underline{388}, 191-210$

Connelly T, Savigner A, Ma M (2013): Spontaneous and sensory-evoked activity in mouse olfactory sensory neurons with defined odorant receptors. J Neurophysiol $\underline{110}, 55$ 62

Corona R, Lévy F (2015): Chemical olfactory signals and parenthood in mammals. Horm Behav 68, 77-90

Cygnar KD, Zhao H (2009): Phosphodiesterase 1C is dispensable for rapid response termination of olfactory sensory neurons. Nat Neurosci 12, 454-462

Czesnik D, Kuduz J, Schild D, Manzini I (2006): ATP activates both receptor and sustentacular supporting cells in the olfactory epithelium of Xenopus laevis tadpoles. Eur J Neurosci 23, 119-128

Dal Monte M, Andreini I, Revoltella R, Pelosi P (1991): Purification and characterization of two odorant-binding proteins from nasal tissue of rabbit and pig. Comp Biochem Physiol Part B Comp Biochem 99, 445-451

Dawson TM, Arriza JL, Jaworsky DE, Borisy FF, Attramadal H, Lefkowitz RJ, Ronnett GV (1993): Beta-Adrenergic receptor kinase-2 and beta-Arrestin-2 as mediators of odorant-induced desensitization. Science 259, 825-830

Doty RL (2012): Olfactory dysfunction in Parkinson disease. Nat Rev Neurol $\underline{8}$, 329-339

Doty RL, Deems DA, Stellar S (1988): Olfactory dysfunction in parkinsonism A general deficit unrelated to neurologic signs, disease stage, or disease duration. Neurology $\underline{38}, 1237-1237$

Duchamp-Viret P, Chaput MA, Duchamp A (1999): Odor Response Properties of Rat Olfactory Receptor Neurons. Science $\underline{284}, 2171-2174$

Duchamp-Viret P, Duchamp A, Chaput MA (2000): Peripheral Odor Coding in the Rat and Frog: Quality and Intensity Specification. J Neurosci 20, 2383-2390

Firestein S (2001): How the olfactory system makes sense of scents. Nature $\underline{413}, 211-218$

Firestein S, Werblin FS (1987): Gated currents in isolated olfactory receptor neurons of the larval tiger salamander. Proc Natl Acad Sci USA 무, 6292-6296

Firestein S, Picco C, Menini A (1993): The relation between stimulus and response in olfactory receptor cells of the tiger salamander. J Physiol 468, 1-10 
Foster WA, Takken W (2004): Nectar-related vs. human-related volatiles: behavioural response and choice by female and male Anopheles gambiae (Diptera: Culicidae) between emergence and first feeding. Bull Entomol Res 94, 145-157

Francia S, Pifferi S, Menini A, Tirindelli R: Vomeronasal Receptors and Signal Transduction in the Vomeronasal Organ of Mammals. In: Mucignat-Caretta (Eds.): Neurobiology of Chemical Communication. CRC Press/Taylor \& Francis, Boca Raton (FL) 2014, Chapter 10

Frenz CT, Hansen A, Dupuis ND, Shultz N, Levinson SR, Finger TE, Dionne VE (2014): $\mathrm{NaV} 1.5$ sodium channel window currents contribute to spontaneous firing in olfactory sensory neurons. J Neurophysiol 112, 1091-1104

Frings S, Lindemann B (1991): Current recording from sensory cilia of olfactory receptor cells in situ. I. The neuronal response to cyclic nucleotides. J Gen Physiol 97, 1-16

Getchell ML, Getchell TV (1992): Fine structural aspects of secretion and extrinsic innervation in the olfactory mucosa. Microsc Res Tech $23,111-127$

Getchell TV (1986): Functional properties of vertebrate olfactory receptor neurons. Physiol $\operatorname{Rev}$ 66, 772-818

Gliem S, Schild D, Manzini I (2009): Highly specific responses to amine odorants of individual olfactory receptor neurons in situ. Eur J Neurosci 29, 2315-2326

Gliem S, Syed AS, Sansone A, Kludt E, Tantalaki E, Hassenklöver T, Korsching SI, Manzini I (2013): Bimodal processing of olfactory information in an amphibian nose: odor responses segregate into a medial and a lateral stream. Cell Mol Life Sci 70, 1965-1984

Goldstein DS, Sewell L (2009): Olfactory dysfunction in pure autonomic failure: Implications for the pathogenesis of Lewy body diseases. Parkinsonism Relat Disord 15, 516-520

Grosmaitre X, Vassalli A, Mombaerts P, Shepherd GM, Ma M (2006): Odorant responses of olfactory sensory neurons expressing the odorant receptor MOR23: A patch clamp analysis in gene-targeted mice. Proc Natl Acad Sci USA 103, 1970-1975

Grüneberg H (1973): A ganglion probably belonging to the $N$. terminalis system in the nasal mucosa of the mouse. Z Anat Entwicklungsgesch 140, 39-52

Hamdani EH, Lastein S, Gregersen F, Døving KB (2008): Seasonal Variations in Olfactory Sensory Neurons-Fish Sensitivity to Sex Pheromones Explained? Chem Senses $\underline{33}, 119-123$

Hansen A, Reiss JO, Gentry CL, Burd GD (1998): Ultrastructure of the olfactory organ in the clawed frog, Xenopus laevis, during larval development and metamorphosis. J Comp Neurol 398, 273-288

Hanson MG, Landmesser LT (2004): Normal Patterns of Spontaneous Activity Are Required for Correct Motor Axon Guidance and the Expression of Specific Guidance Molecules. Neuron 43, 687-701 
Hassenklöver T, Schwartz P, Schild D, Manzini I (2009): Purinergic Signaling Regulates Cell Proliferation of Olfactory Epithelium Progenitors. Stem Cells 27, 2022-2031

Hawkes C (2003): Olfaction in neurodegenerative disorder. Mov Disord 18, 364-372

Hegg CC, Greenwood D, Huang W, Han P, Lucero MT (2003): Activation of Purinergic Receptor Subtypes Modulates Odor Sensitivity. J Neurosci 23, 8291-8301

Hegg CC, Irwin M, Lucero MT (2009): Calcium store-mediated signaling in sustentacular cells of the mouse olfactory epithelium. Glia $\underline{57}$, 634-644

Hubálek Z (2009): Epidemiology of Lyme Borreliosis. Curr Probl Dermatol. 37, 31-50

Junek S, Kludt E, Wolf F, Schild D (2010): Olfactory Coding with Patterns of Response Latencies. Neuron $\underline{67}, 872-884$

Kamath V, Turetsky BI, Calkins ME, Kohler CG, Conroy CG, Borgmann-Winter K, Gatto DE, Gur RE, Moberg PJ (2014): Olfactory processing in schizophrenia, non-ill first-degree family members, and young people at-risk for psychosis. World J Biol Psychiatry 15, 209-218

Kamhawi S (2006): Phlebotomine sand flies and Leishmania parasites: friends or foes? Trends Parasitol 22, 439-445

Kang J, Caprio J (1995): In vivo responses of single olfactory receptor neurons in the channel catfish, Ictalurus punctatus. J Neurophysiol $\underline{73}, 172-177$

Kang J, Caprio J (1997): In Vivo Responses of Single Olfactory Receptor Neurons of Channel Catfish to Binary Mixtures of Amino Acids. J Neurophysiol 7, 1-8

Kato A, Touhara K (2009): Mammalian olfactory receptors: pharmacology, G protein coupling and desensitization. Cell Mol Life Sci $\underline{66}, 3743$

Katz LC, Shatz CJ (1996): Synaptic Activity and the Construction of Cortical Circuits. Science 274, 1133-1138

Kaupp UB (2010): Olfactory signalling in vertebrates and insects: differences and commonalities. Nat Rev Neurosci 11, 188-200

Kludt E, Okom C, Brinkmann A, Schild D (2015): Integrating Temperature with Odor Processing in the Olfactory Bulb. J Neurosci 35, 7892-7902

Lancet D (1986): Vertebrate Olfactory Reception. Annu Rev Neurosci 9, 329-355

Leung CT, Coulombe PA, Reed RR (2007): Contribution of olfactory neural stem cells to tissue maintenance and regeneration. Nat Neurosci 10, 720-726

Lindquist L, Vapalahti O (2008): Tick-borne encephalitis. Lancet $\underline{371}$, 1861-1871

Liu M, Chen T-Y, Ahamed B, Li J, Yau K-W (1994): Calcium-Calmodulin Modulation of the Olfactory Cyclic Nucleotide-Gated Cation Channel. Science 266, 1348-1354

Lledo P-M, Gheusi G, Vincent J-D (2005): Information Processing in the Mammalian Olfactory System. Physiol Rev 모, 281-317 
Lynch JW, Barry PH (1989): Action potentials initiated by single channels opening in a small neuron (rat olfactory receptor). Biophys J $\underline{55}, 755-768$

Ma M, Chen WR, Shepherd GM (1999): Electrophysiological characterization of rat and mouse olfactory receptor neurons from an intact epithelial preparation. J Neurosci Methods $\underline{92}, 31-40$

Ma M, Grosmaitre X, Iwema CL, Baker H, Greer CA, Shepherd GM (2003): Olfactory Signal Transduction in the Mouse Septal Organ. J Neurosci 23 , 317-324

Maddin HC, Eckhart L, Jaeger K, Russell AP, Ghannadan M (2009): The anatomy and development of the claws of Xenopus laevis (Lissamphibia: Anura) reveal alternate pathways of structural evolution in the integument of tetrapods. J Anat 214, 607619

Malnic B, Godfrey PA, Buck LB (2004): The human olfactory receptor gene family. Proc Natl Acad Sci U S A 101, 2584-2589

Manzini I, Schild D (2003): cAMP-independent olfactory transduction of amino acids in Xenopus laevis tadpoles. J Physiol $\underline{51}, 115-123$

Manzini I, Schild D (2004a): Classes and Narrowing Selectivity of Olfactory Receptor Neurons of Xenopus laevis Tadpoles. J Gen Physiol 123, 99-107

Manzini I, Schild D (2004b): Multidrug resistance transporters in the olfactory receptor neurons of Xenopus laevis tadpoles. J Physiol 546, 375-385

Manzini I, Schild D: Olfactory Coding in Larvae of the African Clawed Frog Xenopus laevis. In: Menini A (Ed.): The Neurobiology of Olfaction. CRC Press/Taylor \& Francis, Boca Raton (FL) 2010, Chapter 4

Manzini I, Korsching S (2011): The peripheral olfactory system of vertebrates: molecular, structural and functional basics of the sense of smell. e-Neuroforum $\underline{2}, 68$

Manzini I, Rössler W, Schild D (2002a): cAMP-independent responses of olfactory neurons in Xenopus laevis tadpoles and their projection onto olfactory bulb neurons. J Physiol $\underline{545}$, 475-484

Manzini I, Peters F, Schild D (2002b): Odorant responses of Xenopus laevis tadpole olfactory neurons: a comparison between preparations. J Neurosci Methods $\underline{121}$, 159-167

Manzini I, Brase C, Chen T-W, Schild D (2007): Response profiles to amino acid odorants of olfactory glomeruli in larval Xenopus laevis. J Physiol 581, 567-579

Martín-Sánchez A, McLean L, Beynon RJ, Hurst JL, Ayala G, Lanuza E, Martínez-Garcia F (2015): From sexual attraction to maternal aggression: When pheromones change their behavioural significance. Horm Behav $\underline{68}$, 65-76

Mashukova A, Spehr M, Hatt H, Neuhaus EM (2006): $\beta$-Arrestin2-Mediated Internalization of Mammalian Odorant Receptors. J Neurosci 26, 9902-9912

Menashe I, Aloni R, Lancet D (2006): A probabilistic classifier for olfactory receptor pseudogenes. BMC Bioinformatics $\underline{7}, 393$ 
Menini A., Picco C., Firestein S. (1995): Quantal-like current fluctuations induced by odorants in olfactory receptor cells. Nature $373,435-437$

Meredith M (2001): Human Vomeronasal Organ Function: A Critical Review of Best and Worst Cases. Chem Senses 26, 433-445

Moberg PJ, Agrin R, Gur RE, Gur RC, Turetsky BI, Doty RL (1999): Olfactory Dysfunction in Schizophrenia: A Qualitative and Quantitative Review. Neuropsychopharmacology 21, 325-340

Mombaerts P (2004): Odorant receptor gene choice in olfactory sensory neurons: the one receptor-one neuron hypothesis revisited. Curr Opin Neurobiol 14, 31-36

Mombaerts P (2006): Axonal Wiring in the Mouse Olfactory System. Annu Rev Cell Dev Biol 22, 713-737

Munger SD (2009): Olfaction: Noses within noses. Nature $\underline{459}, 521-522$

Nakagawa T, Vosshall LB (2009): Controversy and consensus: noncanonical signaling mechanisms in the insect olfactory system. Curr Opin Neurobiol 19, 284-292

Nakashima A, Takeuchi H, Imai T, Saito H, Kiyonari H, Abe T, Chen M, Weinstein LS, Yu CR, Storm DR, et al. (2013): Agonist-Independent GPCR Activity Regulates Anterior-Posterior Targeting of Olfactory Sensory Neurons. Cell 154, 1314-1325

Nakashima N, Ishii TM, Bessho Y, Kageyama R, Ohmori H (2013): Hyperpolarisationactivated cyclic nucleotide-gated channels regulate the spontaneous firing rate of olfactory receptor neurons and affect glomerular formation in mice. J Physiol $\underline{591}$, 1749-1769

Nei M, Niimura Y, Nozawa M (2008): The evolution of animal chemosensory receptor gene repertoires: roles of chance and necessity. Nat Rev Genet 9, 951-963

Nene V, Wortman JR, Lawson D, Haas B, Kodira C, Tu Z (Jake), Loftus B, Xi Z, Megy K, Grabherr M, et al. (2007): Genome Sequence of Aedes aegypti, a Major Arbovirus Vector. Science $\underline{316}, 1718-1723$

Nezlin LP, Schild D (2000): Structure of the olfactory bulb in tadpoles of Xenopus laevis. Cell Tissue Res 302, 21-29

Nezlin LP, Schild D (2005): Individual olfactory sensory neurons project into more than one glomerulus in Xenopus laevis tadpole olfactory bulb. J Comp Neurol $\underline{481}, 233$ 239

Nichols M, Townsend N, Scarborough P, Rayner M (2014): Cardiovascular disease in Europe 2014: epidemiological update. Eur Heart J 35, 2950-2959

Nieuwkoop P, Faber J (Eds.) Normal table of Xenopus laevis (Daudin): a systematical and chronological survey of the development from the fertilized egg till the end of metamorphosis. Routledge, New York 1994

Olsen SR, Wilson RI (2008): Lateral presynaptic inhibition mediates gain control in an olfactory circuit. Nature $\underline{452}, 956-960$ 
Papoulis A, Pillai S U: Probability, Random Variables and Stochastic Processes.

$4^{\text {th }}$ Edition; McGraw-Hill Companies, New York 2002

Pause BM, Miranda A, Göder R, Aldenhoff JB, Ferstl R (2001): Reduced olfactory performance in patients with major depression. J Psychiatr Res $\underline{35}, 271-277$

Peppel K, Boekhoff I, McDonald P, Breer H, Caron MG, Lefkowitz RJ (1997): G Proteincoupled Receptor Kinase 3 (GRK3) Gene Disruption Leads to Loss of Odorant Receptor Desensitization. J Biol Chem 272, 25425-25428

Pietra G, Dibattista M, Menini A, Reisert J, Boccaccio A (2016): The Ca2+-activated Clchannel TMEM16B regulates action potential firing and axonal targeting in olfactory sensory neurons. J Gen Physiol 148, 293-311

Polese G, Bertapelle C, Di Cosmo A (2015): Role of olfaction in Octopus vulgaris reproduction. Gen Comp Endocrinol 210, 55-62

Poo C, Isaacson JS (2009): Odor Representations in Olfactory Cortex: "Sparse" Coding, Global Inhibition, and Oscillations. Neuron $\underline{62}, 850-861$

Prud'homme MJ, Lacroix MC, Badonnel K, Gougis S, Baly C, Salesse R, Caillol M (2009): Nutritional status modulates behavioural and olfactory bulb Fos responses to isoamyl acetate or food odour in rats: roles of orexins and leptin. Neuroscience $\underline{162}, 1287-1298$

Pyrski M, Koo JH, Polumuri SK, Ruknudin AM, Margolis JW, Schulze DH, Margolis FL (2007): Sodium/calcium exchanger expression in the mouse and rat olfactory systems. J Comp Neurol 501, 944-958

Reinhard J, Srinivasan MV, Zhang S (2004): Olfaction: Scent-triggered navigation in honeybees. Nature $\underline{427}, 411-411$

Reisert J (2010): Origin of basal activity in mammalian olfactory receptor neurons. J Gen Physiol 136, 529-540

Reisert J, Matthews HR (1998): $\mathrm{Na}^{+}$-dependent $\mathrm{Ca}^{2+}$ Extrusion Governs Response Recovery in Frog Olfactory Receptor Cells. J Gen Physiol 112, 529-535

Ressler KJ, Sullivan SL, Buck LB (1994a): A molecular dissection of spatial patterning in the olfactory system. Curr Opin Neurobiol 4, 588-596

Ressler KJ, Sullivan SL, Buck LB (1994b): Information coding in the olfactory system: Evidence for a stereotyped and highly organized epitope map in the olfactory bulb. Cell $\underline{79}, 1245-1255$

Roberts RO, Christianson TJH, Kremers WK, Mielke MM, Machulda MM, Vassilaki M, Alhurani RE, Geda YE, Knopman DS, Petersen RC (2016): Association Between Olfactory Dysfunction and Amnestic Mild Cognitive Impairment and Alzheimer Disease Dementia. JAMA Neurol 73, 93-101

Rospars J-P, Lánský P, Vaillant J, Duchamp-Viret P, Duchamp A (1994): Spontaneous activity of first- and second-order neurons in the frog olfactory system. Brain Res $\underline{662}, 31-44$ 
Rospars J-P, Sanda P, Lansky P, Duchamp-Viret P (2013): Responses of single neurons and neuronal ensembles in frog first- and second-order olfactory neurons. Brain Res 1536, 144-158

Sansone A, Hassenklöver T, Syed AS, Korsching SI, Manzini I (2014): Phospholipase C and Diacylglycerol Mediate Olfactory Responses to Amino Acids in the Main Olfactory Epithelium of an Amphibian. PLoS One 2 , e87721

Sato K, Pellegrino M, Nakagawa T, Nakagawa T, Vosshall LB, Touhara K (2008): Insect olfactory receptors are heteromeric ligand-gated ion channels. Nature $\underline{452}, 1002$ 1006

Savigner A, Duchamp-Viret P, Grosmaitre X, Chaput M, Garcia S, Ma M, PalouzierPaulignan B (2009): Modulation of Spontaneous and Odorant-Evoked Activity of Rat Olfactory Sensory Neurons by Two Anorectic Peptides, Insulin and Leptin. J Neurophysiol 101, 2898-2906

Schiefner A, Freier R, Eichinger A, Skerra A (2015): Crystal structure of the human odorant binding protein, OBPIIa. Proteins $\underline{83}, 1180-1184$

Schild D, Restrepo D (1998): Transduction Mechanisms in Vertebrate Olfactory Receptor Cells. Physiol Rev $\underline{78}, 429-466$

Serizawa S, Miyamichi K, Takeuchi H, Yamagishi Y, Suzuki M, Sakano H (2006): A Neuronal Identity Code for the Odorant Receptor-Specific and Activity-Dependent Axon Sorting. Cell 127, 1057-1069

Shah M, Muhammed N, Findley LJ, Hawkes CH (2008): Olfactory tests in the diagnosis of essential tremor. Parkinsonism Relat Disord 14, 563-568

Shapiro HA, Zwarenstein H (1934): A Rapid Test for Pregnancy on Xenopus lavis. Nature 133,762

Silbering AF, Benton R (2010): Ionotropic and metabotropic mechanisms in chemoreception: 'chance or design'? EMBO Rep 11, 173-179

Smallegange RC, Knols BGJ, Takken W (2010): Effectiveness of Synthetic Versus Natural Human Volatiles as Attractants for Anopheles gambiae (Diptera: Culicidae) Sensu Stricto. J Med Entomol 47, 338-344

Sourjik V, Wingreen NS (2012): Responding to chemical gradients: bacterial chemotaxis. Curr Opin Cell Biol 24, 262-268

Spehr M, Kelliher KR, Li X-H, Boehm T, Leinders-Zufall T, Zufall F (2006): Essential Role of the Main Olfactory System in Social Recognition of Major Histocompatibility Complex Peptide Ligands. J Neurosci 26, 1961-1970

Spitzer NC (2006): Electrical activity in early neuronal development. Nature 444, 707-712

Spors H, Wachowiak M, Cohen LB, Friedrich RW (2006): Temporal Dynamics and Latency Patterns of Receptor Neuron Input to the Olfactory Bulb. J Neurosci 26, $1247-1259$ 
Stevenson RJ, Langdon R, McGuire J (2011): Olfactory hallucinations in schizophrenia and schizoaffective disorder: A phenomenological survey. Psychiatry Res $\underline{185}, 321-327$

Stopka P, Janotova K, Heyrovsky D (2007): The advertisement role of major urinary proteins in mice. Physiol Behav 91, 667-670

Suzuki Y, Takeda M, Farbman AI (1996): Supporting cells as phagocytes in the olfactory epithelium after bulbectomy. J Comp Neurol 376, 509-517

Syed AS, Sansone A, Nadler W, Manzini I, Korsching SI (2013): Ancestral amphibian v2rs are expressed in the main olfactory epithelium. Proc Natl Acad Sci USA $\underline{110}, 7714$ 7719

Szyszka P, Galizia CG: Olfaction in Insects. In: Doty RL (Ed.): Handbook of Olfaction and Gustation. ${ }^{\text {rd }}$ Edition; John Wiley \& Sons, Inc., Hoboken 2015, 531-546

Takken W, Knols BGJ (1999): Odor-Mediated Behavior of Afrotropical Malaria Mosquitoes. Annu Rev Entomol 44, 131-157

Tegoni M, Pelosi P, Vincent F, Spinelli S, Campanacci V, Grolli S, Ramoni R, Cambillau C (2000): Mammalian odorant binding proteins. Biochim Biophys Acta 1482, 229_ 240

Thornton-Manning JR, Dahl AR (1997): Metabolic capacity of nasal tissue: interspecies comparisons of xenobiotic-metabolizing enzymes. Mutat Res $\underline{380}, 43-59$

Tirindelli R, Dibattista M, Pifferi S, Menini A (2009): From Pheromones to Behavior. Physiol Rev 오, 921-956

Tinsley R., Minter L., Measey J., Howell K., Veloso A., Núñez H. Romano A (2009): Xenopus laevis. The IUCN Red List of Threatened Species 2009:

e.T58174A11730010. https://www.iucnredlist.org/species/58174/11730010 (Access on 16th October 2018)

Treloar H, Walters E, Margolis F, Key B (1996): Olfactory glomeruli are innervated by more than one distinct subset of primary sensory olfactory neurons in mice. J Comp Neurol 367, 550-562

Tritsch NX, Yi E, Gale JE, Glowatzki E, Bergles DE (2007): The origin of spontaneous activity in the developing auditory system. Nature $\underline{450}, 50-55$

Urban NN, Sakmann B (2002): Reciprocal intraglomerular excitation and intra- and interglomerular lateral inhibition between mouse olfactory bulb mitral cells. J Physiol $\underline{542}, 355-367$

van Drongelen W, Holley A, Døving KB (1978): Convergence in the olfactory system: quantitative aspects of odour sensitivity. J Theor Biol $\underline{71}, 39-48$

Vogler C, Schild D (1999): Inhibitory and excitatory responses of olfactory receptor neurons of xenopus laevis tadpoles to stimulation with amino acids. J Exp Biol 202, 997-1003 
Wei J, Zhao AZ, Chan GCK, Baker LP, Impey S, Beavo JA, Storm DR (1998):

Phosphorylation and Inhibition of Olfactory Adenylyl Cyclase by CaM Kinase II in Neurons. Neuron 21, 495-504

Wenning GK, Shephard B, Hawkes C, Petruckevitch A, Lees A, Quinn N (1995): Olfactory function in atypical parkinsonian syndromes. Acta Neurol Scand $\underline{91}$, 247-250

Wicher D, Schäfer R, Bauernfeind R, Stensmyr MC, Heller R, Heinemann SH, Hansson BS (2008): Drosophila odorant receptors are both ligand-gated and cyclic-nucleotideactivated cation channels. Nature $452,1007-1011$

World Health Organization: World Malaria Report 2015. World Health Organization, 2016.

Wong ST, Trinh K, Hacker B, Chan GCK, Lowe G, Gaggar A, Xia Z, Gold GH, Storm DR (2000): Disruption of the Type III Adenylyl Cyclase Gene Leads to Peripheral and Behavioral Anosmia in Transgenic Mice. Neuron 27, 487-497

Yu CR, Power J, Barnea G, O’Donnell S, Brown HEV, Osborne J, Axel R, Gogos JA (2004): Spontaneous Neural Activity Is Required for the Establishment and Maintenance of the Olfactory Sensory Map. Neuron 42, 553-566

Zancanaro C: Vomeronasal Organ: A Short History of Discovery and an Account of Development and Morphology in the Mouse. In: Mucignat-Caretta C (Eds.): Neurobiology of Chemical Communication. CRC Press/Taylor \& Francis, Boca Raton (FL) 2014

Zielinski BS, Hara TJ (2006): Olfaction. Fish Physiol 로, 1-43

Zwiebel LJ, Takken W (2004): Olfactory regulation of mosquito-host interactions. Insect Biochem Mol Biol 34, 645-652 


\section{Danksagung}

Mein besonderer Dank gilt Herrn Prof. Dr. Dr. Detlev Schild, der mir die Gelegenheit gab, in seinem Labor das spannende Projekt zu bearbeiten. Alle gemeinsamen Gespräche zum Projekt sowie im Allgemeinen hatten eine inspirierende Wirkung und waren stets eine Motivation auch kritische Phasen zu überstehen.

Weiterhin möchte ich besonders Dr. Guobin Bao für seine Unterstützung bei der Analyse und Interpretation der Rohdaten mittels MatLab-Skripten danken. Seine zahlreichen Anmerkungen und Anregungen waren sehr hilfreich und essentielle Bausteine zum Gelingen des Projektes.

Weiterhin bedanke ich mich bei Dr. Camille Okom für das kritische Korrekturlesen der Endfassung meiner Arbeit. Die zahlreichen Kommentare waren eine große Hilfe bei der Anfertigung dieser Endversion!

Schließlich möchte ich mich auch bei meinen anderen Kollegen aus dem Labor, namentlich Dr. Jokob Eifler, Dr. Mihai Alevra, Dr. Danielle de Jong, Dr. Alexander Brinkmann und meinem Mitdoktoranden Erik Rauf, für die kollegiale und freundliche Atmosphäre sowie zahlreichen Diskussionen und Kommentare, ob zum Projekt oder zu Themen der Allgemeinwissenschaft, bedanken. Darüber hinaus möchte ich mich bei Danielle, Mihai und Erik für die gute Zusammenarbeit im Rahmen unseres gemeinsamen Projektes „Resolving different presynaptic activity patterns within single olfactory glomeruli of Xenopus laevis larvae" bedanken.

Abschließend bedanke ich mich bei Christina Petzold für die technische Assistenz und Unterstützung im Labor. 